University of Louisville

ThinkIR: The University of Louisville's Institutional Repository

Electronic Theses and Dissertations

$5-2013$

\title{
Cardiovascular disease among diabetic women with bilateral oophorectomy.
}

Duke Appiah

University of Louisville

Follow this and additional works at: https://ir.library.louisville.edu/etd

Part of the Epidemiology Commons

\section{Recommended Citation}

Appiah, Duke, "Cardiovascular disease among diabetic women with bilateral oophorectomy." (2013). Electronic Theses and Dissertations. Paper 43.

https://doi.org/10.18297/etd/43

This Doctoral Dissertation is brought to you for free and open access by ThinkIR: The University of Louisville's Institutional Repository. It has been accepted for inclusion in Electronic Theses and Dissertations by an authorized administrator of ThinkIR: The University of Louisville's Institutional Repository. This title appears here courtesy of the author, who has retained all other copyrights. For more information, please contact thinkir@louisville.edu. 


\title{
CARDIOVASCULAR DISEASE AMONG DIABETIC WOMEN WITH BILATERAL OOPHORECTOMY
}

\author{
By \\ Duke Appiah \\ BSc., Kwame Nkrumah University of Science and Technology, 2005 \\ M.P.H., University of Louisville, 2009
}

\begin{abstract}
A Dissertation
Submitted To the Faculty Of The

School of Public Health and Information Sciences of the University Of Louisville in Partial Fulfillment of the Requirements

for the Degree of
\end{abstract}

Doctor of Philosophy

Department of Epidemiology and Population Health

University of Louisville

Louisville, Kentucky

May 2013 
Copyright 2013 by Duke Appiah

All rights reserved 



\title{
CARDIOVASCULAR DISEASE AMONG DIABETIC WOMEN WITH BILATERAL OOPHORECTOMY
}

\author{
By \\ Duke Appiah \\ BSc., Kwame Nkrumah University of Science and Technology, 2005 \\ M.P.H., University of Louisville, 2009
}

A Dissertation Approved on

April 16, 2013

By the following Dissertation Committee:

Carlton A. Hornung, Ph.D., M.P.H. (Chair)

Stephen J. Winters, M.D.

Richard N. Baumgartner, Ph.D.

Matthew A. Allison, M.D., M.P.H.

Frank D. Groves, M.D., M.P.H.

John Myers, Ph.D., M.S.P.H. 


\section{ACKNOWLEDGEMENTS}

Blessed be the name of the lord Jesus Christ who according to his divine power has enabled me to complete my doctoral study. Praise forever be unto his name for strengthening me throughout all the changing scenes of life. I love you Lord.

This dissertation could have never been possible without the guidance, encourage, and support, from many professors, family and loved ones. I am especially grateful and thankful to my committee chair Dr. Carlton Hornung for his motivation, devotion, guidance, and for always believing in me even when I doubted my own abilities. His expert knowledge in cardiovascular epidemiology allowed me to complete my doctoral study in a timely manner. You are unique and will always be cherished. I'm forever grateful and hope to become a wonderful professor like you someday.

Gratitude and thanksgiving is also extended to all the committee members (Drs. Winters, Baumgartner, Allison, Groves and Myers) who devoted their time to me whenever I needed them and for their kindness and patience in explaining key concepts to me which made the dissertation a success. I would also like to acknowledge and thank Drs. Jane Cauley and Susan Muldoon who sponsored me to gain access to the study of Osteoporotic Fractures which was used in this dissertation.

I owe a debt of gratitude to all the wonderful faculty of the departments of epidemiology and biostatistics for their great tutelage. 
Your contributions to my professional growth are invaluable and it was an honor to have had the opportunity to learn from you all. Many kind appreciations go to Dr. Harris for her outstanding kindness, encouragement, knowledge and care. I would also like to express my profound gratitude to Ms. Tammi A. Thomas for her ever present help and administrative support.

Finally, I will like to acknowledge the network of family and loved ones whose constant love and comfort have guided me through my doctoral training. It is with heartfelt gratitude and love that I thank my beautiful beloved virtuous wife Linda for being there time after time, always going the extra mile with her kind and thoughtful spirit. To my dear parents and siblings (Mr. Anthony Appiah, Mrs. Sarah Eshun-Appiah, Antonitta and Allan), and to my cherished adapted Kentucky family (Dr. Vanita Farrow, Dr. J. P. Skinner and the late Dr. Marilyn Brown-Anderson) who all kept me supplied with lots of prayer support and infinite love. I say thank you so very much for everything that you did to bring me to the pinnacle of education. I truly appreciate you all greatly. God bless each and everyone who loved me enough to walk alongside me during this formidable and exciting journey. 


\section{ABSTRACT \\ CARDIOVASCULAR DISEASE IN DIABETIC WOMEN WITH BILATERAL OOPHORECTOMY}

Duke Appiah

April 16, 2013

Cardiovascular Disease (CVD) is the leading cause of death in women and the risk is increased threefold in diabetics. In postmenopausal diabetics, the ovary responds to hyperinsulinemia by increasing secretion of testosterone precursors which increases the risk of CVD.

Data from the National Health and Nutrition Examination Survey (1988-2010) and the Study of Osteoporotic Fractures were used to test the hypothesis that a history of bilateral oophorectomy -surgical removal of both ovaries-would decrease the risk of CVD among postmenopausal diabetic women due to reduced androgen levels. Logistic regression, discrete time logit model, B-Spline regression and Cox proportional hazards models were performed with adjusted estimates and 95\% confidence intervals (CIs) calculated.

Overall, the studies comprised of 17,549 postmenopausal women with approximately $17.1 \%$ having a history of diabetes Mellitus. Additionally, $24.1 \%$ of diabetic women had undergone bilateral oophorectomy with $55 \%$ of these having the surgery before age 45 years. Diabetics were more likely to be obese, live a more sedentary lifestyle, have dyslipidemia and were more likely to report a higher prevalence 
of stroke and myocardial infarction than non diabetics at baseline. Women who had oophorectomy before age 45 years were more likely to be nulliparous and report a family history of myocardial infarction.

Diabetic women with oophorectomy had lower levels of total testosterone compared to diabetic women with intact ovaries or naturally menopausal non-diabetic women, which was independent of age and body mass index $(\mathrm{p}=0.016)$. In multivariable regression models, bilateral oophorectomy was predictive of prevalent CVD in diabetics (Odds ratio: 1.63 (95\% CI: $1.16-2.30)$ with the odds limited to women who had this procedure before age 45 years (OR: 2.11, CI: 1.45-3.08). Although ovarian status did not influence incident CVD in diabetic women (OR: 0.78, CI: 0.56-1.09), women with oophorectomy before the average age at menopause (51 years), with or without diabetes were observed to have elevated CVD risk in spline regression models. Furthermore, the risk of CVD mortality was elevated for diabetic women irrespective of ovarian status, although women with no ovaries had an increased risk (Hazard ratio: 2.57, CI: 1.18-3.67) than those with intact ovaries (HR: 1.99, CI: 1.65-2.39).

This present analyses consisting of three different population-based samples of postmenopausal women did not support oophorectomy having a protective effect on cardiovascular health in diabetic women; however, our interpretation of these data is that oophorectomy was performed more often in younger women who inherited a risk factor for heart disease. 


\section{TABLE OF CONTENTS}

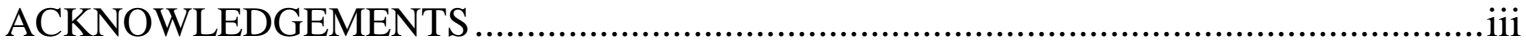

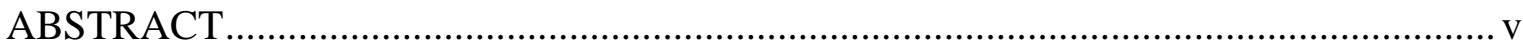

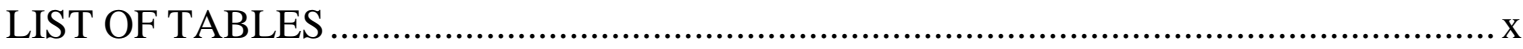

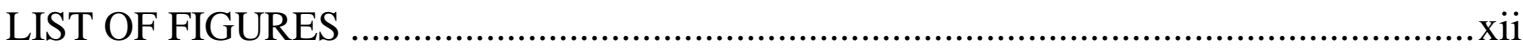

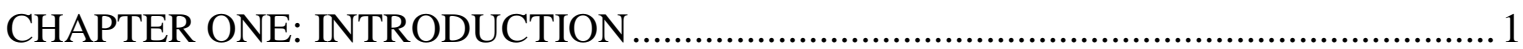

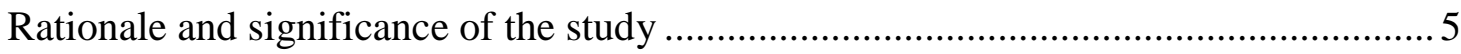

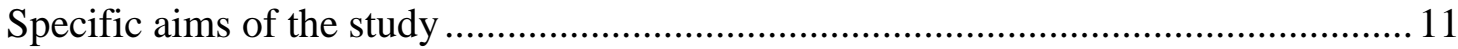

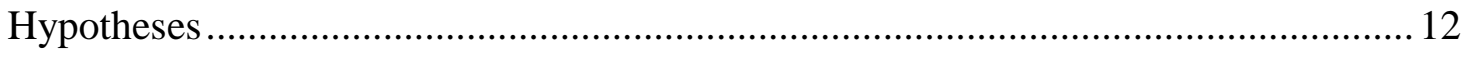

CHAPTER TWO: BACKGROUND AND SIGNIFICANCE ...................................... 13

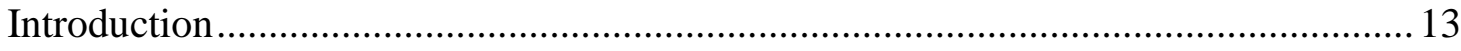

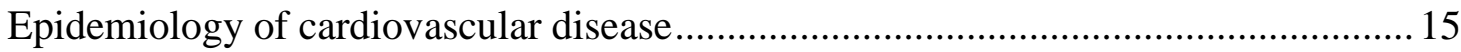

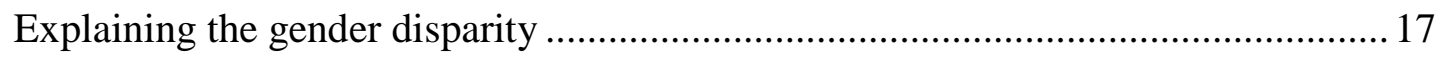

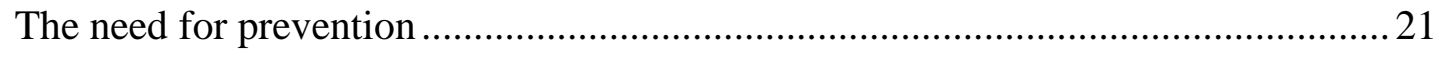

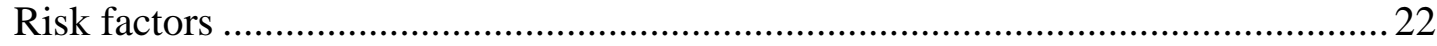

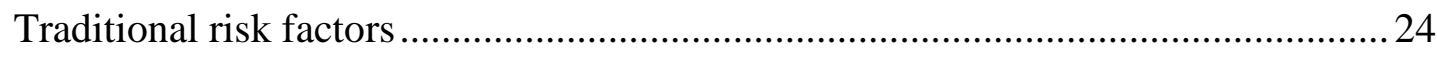

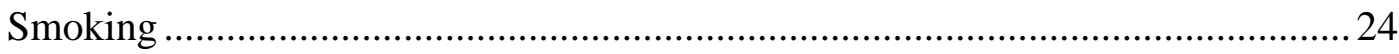

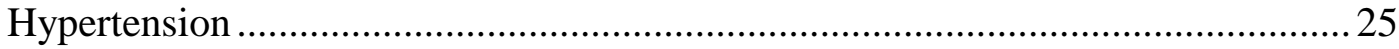

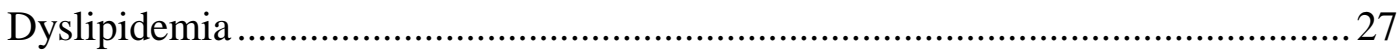

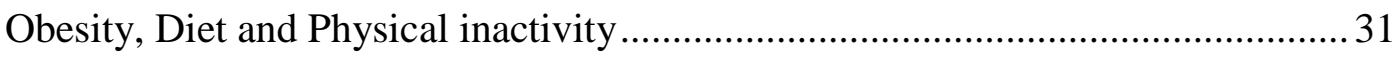

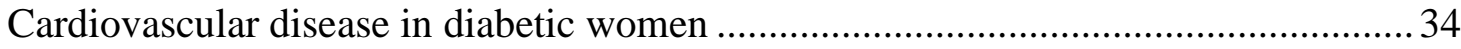

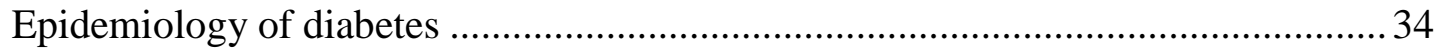

The Impact of Cardiovascular Disease in Diabetic women ................................... 35

Pathophysiology of diabetes and cardiovascular disease ...................................... 37 
The effect of sex-specific characteristics on CVD in women................................ 48

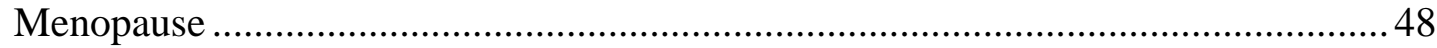

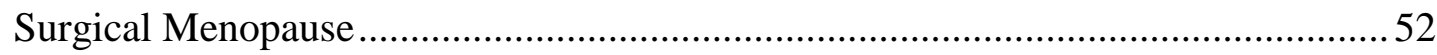

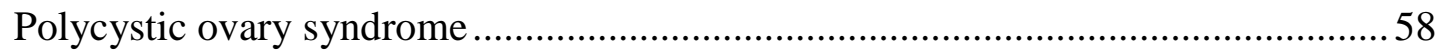

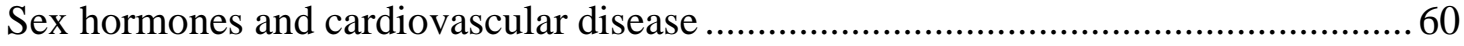

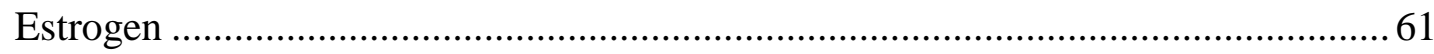

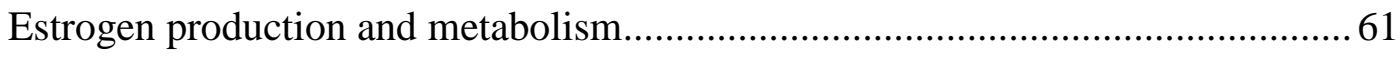

Protective Effects of Estrogen on the Cardiovascular System.............................62

Hormone therapy and cardiovascular disease .............................................. 63

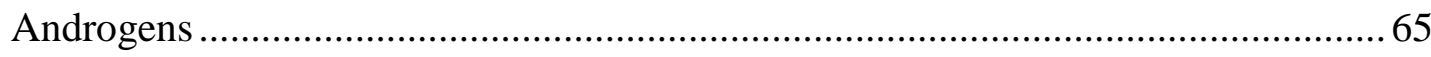

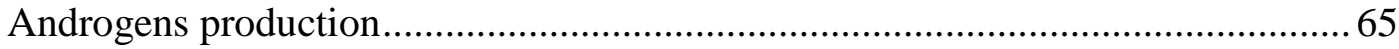

The Effect of Androgens on Cardiometabolic risk factors ...................................69

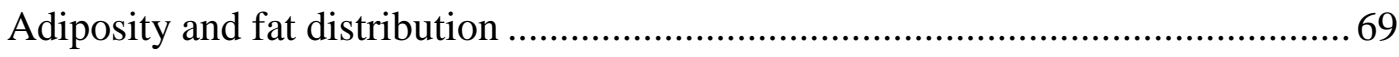

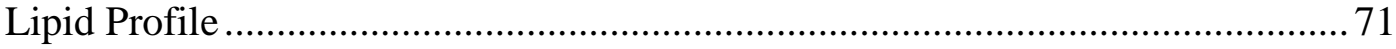

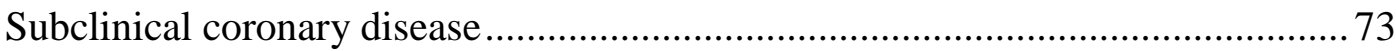

The Effect of Androgens on Cardiovascular disease outcomes............................... 74

The Effect of Androgens on Cardiovascular disease in diabetic women ................. 75

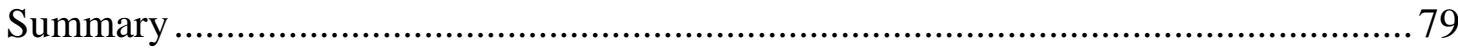

CHAPTER THREE: RESEARCH DESIGN AND METHODS ................................... 81

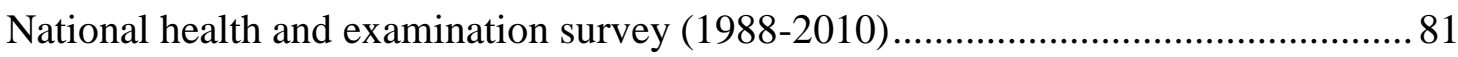

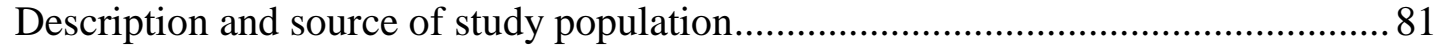

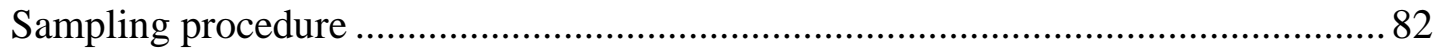

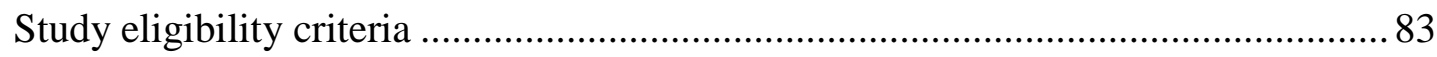

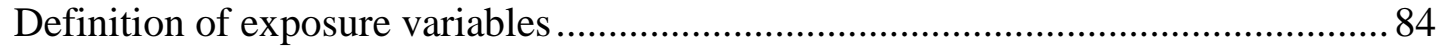

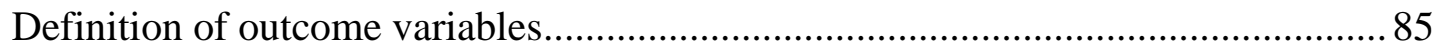

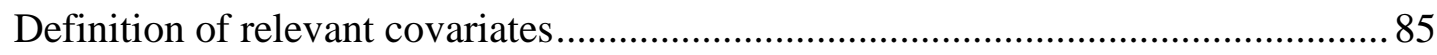

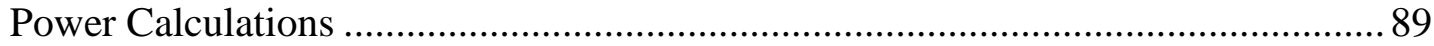

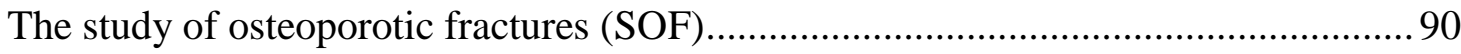

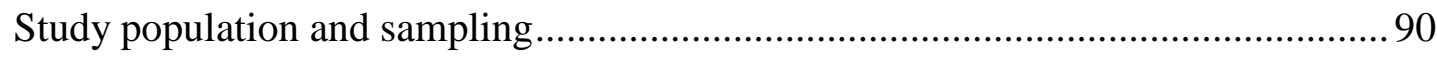

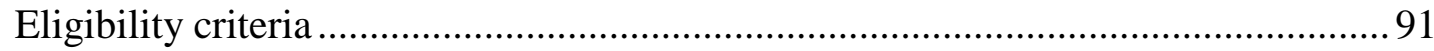

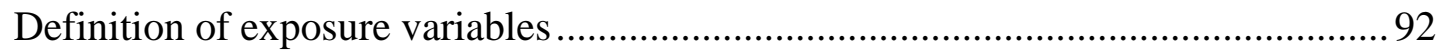




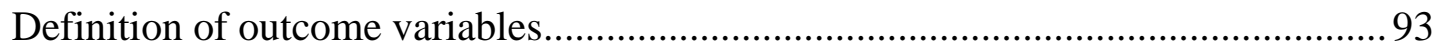

Definition of relevant covariates............................................................................ 93

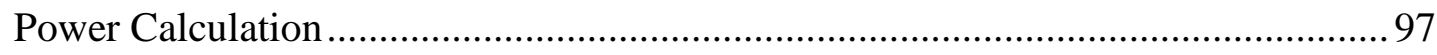

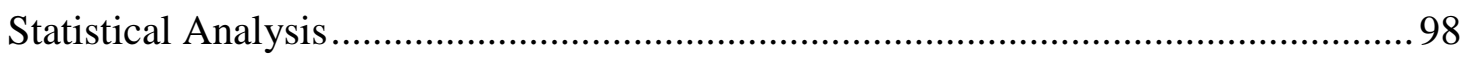

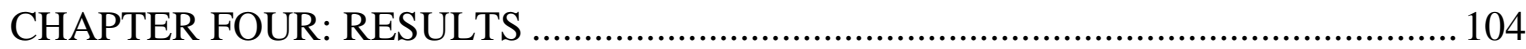

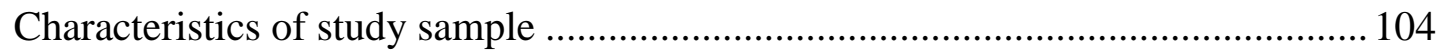

Sex-steroid hormones levels according to diabetes and oophorectomy status ......... 117

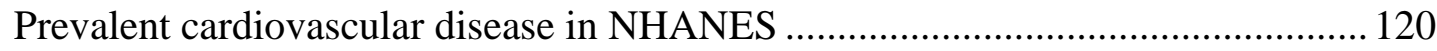

Incident cardiovascular disease in SOF study ......................................................... 124

Age at natural or surgical menopause and risk of incident CVD in SOF study ...... 126

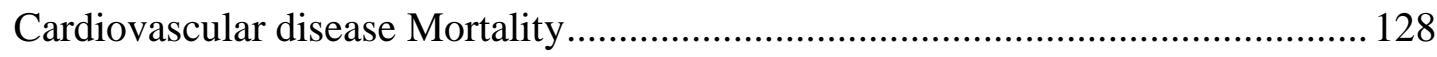

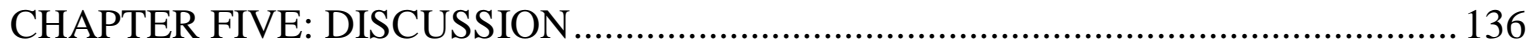

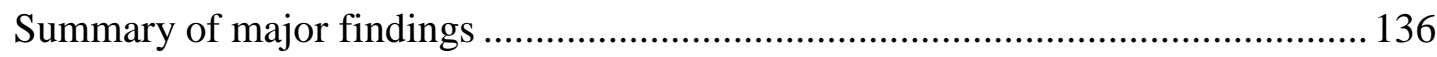

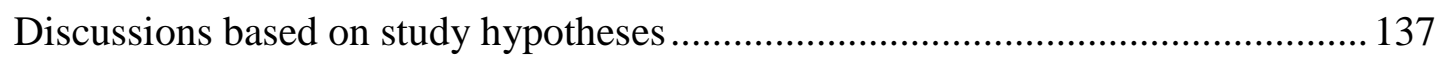

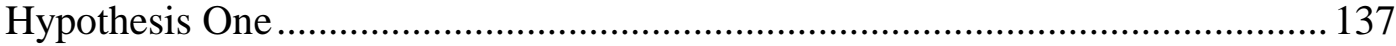

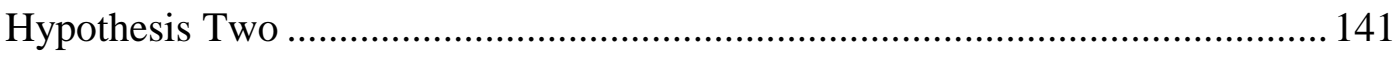

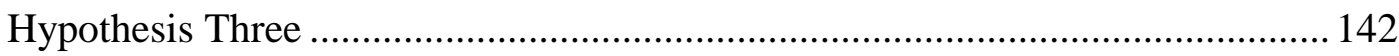

Addressing the conundrum about oophorectomy and women's long term health... 148

Strengths and limitations..................................................................................... 150

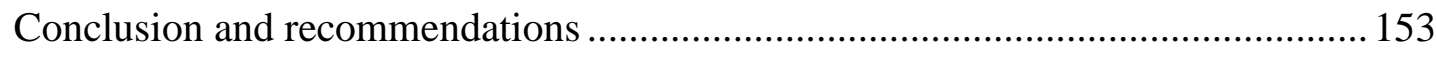

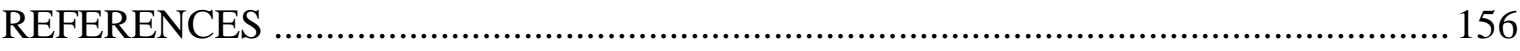

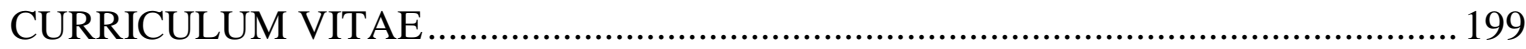




\section{LIST OF TABLES}

Table 2.1: Disorders of Glycemic Homeostasis .43

Table 2.2: Studies of endogenous testosterone and risk of cardiovascular disease (CVD) outcomes in postmenopausal women

Table 4.1: Characteristics of study population, the third National Health and Nutrition Examination Survey (1988-1994)

Table 4.2: Characteristics of study population, National Health and Nutrition Examination Survey (1999-2010)

Table 4.3: Characteristics of diabetic women according to age at oophorectomy, National Health and Nutrition Examination Survey (1999-2010) 112

Table 4.4: Characteristics of non-diabetic women according to age at oophorectomy, National Health and Nutrition Examination Survey (1999-2010) 113

Table 4.5: Baseline characteristic of 7,950 postmenopausal women according to diabetes and bilateral oophorectomy status, The Study of Osteoporotic Fractures 115

Table 4.6: Hormone levels [median (IQR)] according to diabetes and bilateral oophorectomy status, the Study of Osteoporotic Fractures 118

Table 4.7: Association of free testosterone, total testosterone and dehydroepiandrosterone sulfate and risk of CVD mortality, the Study of Osteoporotic Fractures 119 
Table 4.8: Odds of prevalent cardiovascular disease according to diabetes and oophorectomy status, Third National Health and Nutrition Examination Survey (1988-1994)

Table 4.9: Odds of prevalent cardiovascular disease according to diabetes and oophorectomy status, National Health and Nutrition Examination Survey (19992010)

Table 4.10: Odds of prevalent cardiovascular disease among diabetics by age at oophorectomy, National Health and Nutrition Examination Survey (1999-2010) .....123

Table 4.11: The risk of incident non-fatal cardiovascular disease according to diabetes and bilateral oophorectomy status, the Study of Osteoporotic Fractures

Table 4.12: Incidence of cardiovascular disease related mortality, NHANES III (19881994)

Table 4.13: The risk of cardiovascular disease related mortality according to diabetes and bilateral oophorectomy status, NHANES III (1988-1994) 129

Table 4.14: The risk of all cause and cardiovascular disease related mortality according to diabetes and bilateral oophorectomy status, the Study of Osteoporotic Fractures......132 


\section{LIST OF FIGURES}

Figure 2.1: The mechanism of menopause and cardiovascular disease............................54

Figure 2.2: Sources of testosterone production in postmenopausal women ......................68

Figure 2.3: Actions of elevated testosterone on cardiovascular risk and disease ...............70

Figure 3.1: Detectable alternative relative risk based on a fixed sample size in the SOF ...98

Figure 4.1: Odds ratio and 95\%CI estimates for age at menopause and the incidence of cardiovascular disease, the Study of Osteoporotic Fractures

Figure 4.2: Odds ratio and 95\%CI estimates for age at bilateral oophorectomy and the incidence of cardiovascular disease, the Study of Osteoporotic Fractures

Figure 4.3: Kaplan-Meier cumulative incidence estimates of fatal CVD events among participants without a history of cardiovascular disease, NHANES (1988-1994) ....130

Figure 4.4: Kaplan-Meier cumulative incidence estimates of CVD death in participants without a history of heart disease, The Study of Osteoporotic Fractures

Figure 4.5: Summary estimates of the risk of cardiovascular disease according to diabetes and oophorectomy status

Figure 4.6: Summary estimates of the risk of cardiovascular disease in women with diabetes according to oophorectomy status as well as age at oophorectomy

Figure 4.7 Summary estimates of the risk of cardiovascular disease in women according to age at oophorectomy 


\section{CHAPTER ONE}

\section{INTRODUCTION}

Type 2 diabetes is a metabolic disorder that is characterized by high blood glucose levels and either insufficient insulin production or ineffective insulin action on target cells (1). According to the International Diabetes Federation, 366 million (8.3\%) adults worldwide are living with type 2 diabetes mellitus, and this number is projected to increase to 552 million (9.9\%) by the year 2030; a number that has been attributed to the concurrent obesity epidemic (2). In the United States, diabetes is the seventh leading cause of death (3), and among diabetics aged 65 and older, heart disease accounts for $68 \%$ of all deaths (3). More than 12.6 million (11\%) U.S. women over the age of 20 years are estimated to have diabetes (4), and diabetes is thought to be a stronger risk factor for CHD and cardiovascular disease (CVD) as whole, in women than men (5).

Women with diabetes are more likely to die of CHD compared to non diabetic women or men with and without diabetes (5). Furthermore, diabetes has more debilitating effects in women than men (6-9). For example, the estimate of mortality attributable to CHD in men is 1 to 3 fold higher in diabetic than non-diabetic men while the risk in women is 2 to 5 fold (8).

Additionally, the risk of CVD related-mortality among diabetic women with a 
prior history of CHD is 14 fold compared to 6.6 fold increased risk for women with a prior history of CHD but no diabetes (10). Although women generally have poor outcomes after coronary interventions namely percutaneous transluminal coronary angioplasty (PTCA) and coronary artery bypass grafting (CABG), diabetic women are reported to have far greater adverse outcomes compared to diabetic men or non-diabetic women of same age (11).

Before menopause, women are 50\% less likely to have a CVD event than men of similar age. For example, among premenopausal women aged 35 to 39 in England and Wales, the death rate from CHD was 30 per million compared to 194 per million persons for men of same age (12). Diabetes eliminates the premenopausal gender related differences in the prevalence of CHD $(5,7)$. In postmenopausal women, diabetes increases the risk of a CHD event threefold compared to those without diabetes $(5,10$, 13). Older diabetic women without CVD have a similar risk of cardiovascular mortality compared to non-diabetic women with pre-existing CVD (14). Reasons why diabetes increases CVD risk markedly in women compared to non-diabetic women is not well understood. However, diabetes may induce a more adverse cardiovascular risk profile and this may partially explain the greater excess CVD risk seem in them compared to non-diabetic women (15). Traditional risk factors for CVD such as cholesterol, blood pressure, and smoking which are also prevalent in diabetics do not fully explain the gender difference in risk $(6,9)$.

While the loss of estrogens was previously thought to be the cause of the increased CVD risk in women across the lifespan, current epidemiologic evidence suggests that factors associated with the menopausal transition such as changes in 
cardiometabolic risk factors (hyperglycemia, dyslipidemia, insulin resistance and abdominal obesity) play important roles and might rather explain the increasing CVD risk (16-20). The poor CVD outcomes in diabetic women which maybe a reflection of underdiagnosis or treatment bias that favors men (15), should also not be overlooked. In most industrialized countries, the rate of mortality attributable to cardiovascular diseases (CVD), namely coronary heart disease (CHD), has been declining over the last 50 years. Since 1960, when mortality rates for CVD in the United States were at their highest, a steady decrease has been observed which is believed to be a reflection of the progress made in primary prevention especially smoking cessation, as well as better diagnosis aided by novel imaging techniques, and finally improved treatment due to great advances in thrombolytic therapy, pharmaceutical agents and interventional devices such as percutaneous transluminal coronary angioplasty with a drug-eluting stent and coronary artery bypass graft $(21,22)$. As an example, from the period of 1998 to 2008 , national estimates for CVD related mortality declined by $30.6 \%$ (23). Despite this decline, cardiovascular disease related mortality accounts for $32.8 \%$ of all deaths (24).

CVD is the leading cause of morbidity and mortality in women, and more than a quarter of a million women aged 50 - 79 years in the United States die of heart diseases each year (25). Contrary to popular belief, CHD claims more deaths compared to cancer, chronic lower respiratory diseases and Alzheimer disease combined $(23,24)$. In fact, compared to 1 in 31 deaths due to breast cancer, CHD results in 1 in 6.6 deaths in women $(23,24)$. While high, these rates are projected to increase in the coming decade due to the 
debilitating effects of diabetes and obesity, coupled with an increasing aging population (26).

It clearly is of substantial importance to understand the factors that contribute to the gender disparity in CVD incidence and mortality. While some studies have reported a gender bias in the utilization of percutaneous coronary intervention and CABG procedures, others have found no such bias after correcting for age, differences in severity of disease at time of surgery and comorbidities (27). Gender disparities, however, exist in both medical care and outcomes of treatment procedures. Women account for only $33 \%$ of the 1.2 million coronary interventions performed annually nationwide (28). Additionally, women are less likely than men to receive the recommended cardiovascular standards of care (29), undergo extensive evaluation of cardiac symptoms (30) and be prescribed common prevention pharmaceutical agents (such as aspirin, beta blockers, heparin, statins) (31). They also receive delayed thrombolytic therapy (31). Furthermore, with regards to high-risk patients, as classified by Thrombolysis in Myocardial Infarction (TIMI) scores, only 47.6\% of women received angiograms, angioplasty and CABG procedures compared to $60.5 \%$ of men $(31,32)$. Additionally, women who have both CVD and other comorbidities such as diabetes and dyslipidemia are less likely to receive frequent prescriptions to control these comorbidities $(31,33)$. As highlighted earlier, this may well explain a large component of the excess risk in cardiovascular disease related mortality associated with diabetes in women compared to men (15) 


\section{RATIONALE AND SIGNIFICANCE OF THE STUDY}

While the epidemiology, symptoms and prognosis of CVD differs in women and men, the basis for these differences is not well understood. In addition, CVD risk factors seem to have different impacts on the incidence of disease in the two sexes. For instance, while cholesterol is a major predictor of disease in men, diabetes and hypertension are more predictive of CVD in women (34). Moreover, the roles of sex specific characteristics, such as endogenous hormones (androgens and estrogens), pregnancy and menopause, together with genetic mechanisms and comorbidities, such as diabetes, in the risk of CVD in women are not well understood.

Women on average develop CVD when they are about 10 years older than men, and typically after the onset of menopause (34). Menopause is the transitional period after which the ovaries stop producing eggs, and there is a cessation of menstruation. During this period the levels of both estrogens and androgens are lower than in premenopausal women due to follicular depletion and age-related atrophy of the adrenal cortex (35). However, the postmenopausal ovaries remain hormonally active, secreting significant amounts of androgens (35). In women with bilateral salpingo-oophorectomy (BSO), which is the surgical removal of the ovaries, the levels of androgens are lower than in women with natural menopause $(36,37)$. This was confirmed by a recent study using liquid chromatography-tandem mass spectrometry (38). Contrary to the idea that the majority of the testosterone produced by the postmenopausal ovary is converted into estradiol thereby resulting in no significant difference in testosterone levels between women with and without ovaries, testosterone is approximately $50 \%$ lower in women 
with BSO than naturally menopausal women with this difference persisting throughout the menopausal years (39).

Understanding the influence of the hormonal milieu at menopause on cardiovascular health remains elusive (40). Furthermore, there exists a debate in the literature as to whether chronological aging or biological ovarian aging (follicular oocyte depletion resulting in menopause which also causes reduced ovarian estrogen production) is the reason for of the increased CVD risk in postmenopausal women compared to premenopausal women, and for the delayed in CVD onset in women compared to men (12). Depending on the type of statistical analyses employed, a rise in coronary disease risk after menopause may or may not be observed. A rise/inflection in coronary disease risk after menopause depicts ovarian aging contributing to CVD risk while no rise in coronary disease risk at or after menopause gives evidence to chronological age predisposing women to CVD. Displaying crude CHD rates compared with age-specific rates or male to female age-specific death rates or semi-logarithmic graphs alternatively support the idea that menopause or chronological aging affect CHD risk (41). Such discrepancies have caused many to argue that the increase or no increase in CVD risk after menopause maybe an artifact of the way vital statistics data are presented (41).

Studies of women with early natural menopause and CVD risk present equivocal results. Although inadequate control for smoking (which is known to decrease the age of natural menopause by 2 years) can confound results, most (41-45) but not all $(46,47)$ studies which made adequate adjustment for current smoking status still found an association between early natural menopause and CVD risk. Interestingly, the Framingham Heart Study cohort reported that premenopausal CVD risk factors (smoking, 
increased weight, blood pressure and decreased total cholesterol levels) determines age at natural menopause with the authors concluding that such observations offers novel explanation for the inconsistent findings on cardiovascular disease rate and its relationship to menopausal age (48).

Hysterectomy is the second most common surgery performed on women of childbearing age after Cesarean section, and more than half of all women having a hysterectomy also have both ovaries removed (BSO) most often as primary prevention for ovarian cancer (49). But bilateral oophorectomy eliminates ovarian hormones, and might be expected to influence CAD risk. Women who undergo hysterectomy (regardless of oophorectomy status) have an adverse CVD risk profile compared to those without hysterectomy, and BSO is linked to increasing CVD risk in some $(41,50-54)$ but not all studies (55-59). However, the increased CVD risk among women with BSO is limited to women who have the procedure before the average age at natural menopause (or an arbitrary age of 45 years) and those not receiving hormone therapy (51-54). Methodological issues, namely inappropriate comparison groups, small sample size and lack of multivariable models have contributed to the conflicting results concerning how BSO affects CVD $(57,59)$.

Assuming that the postmenopausal estrogen decline predisposes to increased CVD risk, then hormone therapy (HT) during menopause would be expected to reduce this risk. However, controlled clinical trials have refuted previously accepted conclusions from observational studies that exogenous hormones are protective in the primary prevention of heart diseases (60-62). The use of postmenopausal HT, which has been suggested to have a short term beneficial effect when initiated immediately after BSO in 
premenopausal women (63), does not provide any protective effect against CVD in women who have BSO after the expected natural age at menopause (64). Furthermore, data from controlled trials provides little or no support for the timing hypothesis of favorable effects from CVD among women who initiate postmenopausal HT soon after menopause, either in women with hysterectomy only or intact uterus (65).

The failure to demonstrate a protective effect of estrogens in the primary prevention of CVD in clinical trials has brought an increasing scientific interest how thyroid-stimulating hormone and BSO are related to CVD risk, and to the possible role of androgens and insulin resistance. Results from the largest U.S study on the menopausal transition based on 10 years of follow-up indicate that changes in the lipid profile during menopause tend to favor the progression of atherosclerosis (40). Across women of different ethnicity, total cholesterol, low-density lipoprotein (LDL) cholesterol and apolipoprotein-B levels increase during the menopause (40), while variation in HDL subclass is observed with a decrease in HDL2 levels concurrent with an increase in HDL3 level (66). Additionally, an increase in visceral adiposity during this transitional period has been noted (67). These changes in the lipid milieu and adiposity result in a more atherogenic profile. Moreover, women with visceral adiposity have increased endogenous androgens (67) which increase levels of total and LDL cholesterol, reduce HDL cholesterol and increase lipogenesis (68-70). Visceral fat may directly influence atherosclerosis by accelerating the progression of coronary calcification (71).

Not only is there is much greater decline in estradiol than in testosterone production following menopause, but also the effect of estrogen to increase sex hormone binding globulin (SHBG) and thereby decrease the free bioactive component of 
androgens in circulation falls (72). Therefore the failure of estrogens to oppose the activity of testosterone during the menopausal transition results in a state of relative androgen excess (73). As testosterone progressively dominate the hormonal milieu during the menopausal transition, the occurrence of other cardiometabolic disorders as well as the effect of visceral adipose tissue (VAT) increase, independent of age (74). For instance the Study of Women's Health Across the Nation (SWAN) established that the risk of developing metabolic syndrome was associated with an increase in bioavailable testosterone or a decrease in sex hormone-binding globulin (SHBG) levels (74). Postmenopausal women with diabetes are especially susceptible to CVD. While HT was found to reduce the incidence of diabetes (75-77), other trials enrolling diabetics reported that even though HT improved lipid profile of diabetic women, it however predisposed them to a faster progression of coronary atherosclerosis (78).

Testosterone levels are higher in postmenopausal women with diabetes than in non-diabetic controls either matched on age and body mass index (BMI) (79-81), age and years since menopause (82), or regression adjustment for age, BMI and waist to hip ratio $(83,84)$. Even among diabetic women with similar total fat mass, android adiposity, waist circumference and upper body fat as nondiabetics, the same relationship is observed (85). These results tend to suggest that independent of age or adiposity; hyperandrogenemia may play a role in the link between diabetes and CVD in women and may as well explain the gender difference in diabetes-associated CVD $(36,82,86)$. Lower sex-hormone binding globulin (SHBG) levels in diabetics further increase the circulating non-SHBG bound (bioavailable) testosterone and this further amplifies the effect of testosterone independent of overall adiposity (body mass index) and central 
adiposity (as measured by the ratio of waist to hip circumferences) (87). Although some studies have found low levels of SHBG and high androgenicity to be associated with dyslipidemia and increased carotid artery intima-media thickness, which in turn is associated with an adverse CVD risk factor profile in postmenopausal women (88-90), evidence for an association with hard cardiovascular endpoints especially among diabetic women in longitudinal studies is lacking. While the source of the excess androgens in diabetic women has not been studied in detail, results in women with polycystic ovary syndrome (PCOS), who are also more likely to be obese and insulin resistant $(91,92)$, suggest an ovarian source of androgens in diabetics, although increased adrenal steroid production might also contribute in part to the higher androgen levels reported in diabetic women (93). Moreover, the ovaries in diabetics may have a reduced ability to convert androgens to estrogens due to a reduction in ovarian aromatase activity (94).

Based on these observations, if increased testosterone levels raise the risk for CVD in diabetic women, then reducing androgens should decrease this risk. A means of assessing this hypothesis is by studying women with BSO.

An alternative possibility is that a sizeable portion of women whose ovaries are removed are prediabetic with insulin resistance and anovulation, representing a group of women with increased CVD risk (95) independent of ovarian hormones, and that BS0 in these women will produce the same outcomes as they grow older and develop diabetes as in nondiabetics. Furthermore, if estrogens limit visceral fat accumulation, and thereby insulin resistance, then the removal of the ovaries at an early age may have a more adverse effect than if this procedure is performed after the time of natural menopause. 
CVD is a complex process that includes genetic, inflammatory, and immune factors as well as endocrine components. An added complexity is the influence of diabetes on cardiovascular health especially among women. A great deal of research has been done to improve our understanding regarding the roles cardiovascular risk factors play to influence disease as well as interaction between these factors. Despite numerous investigations into the moderating effect of gender on the etiology of CVD, as well as improving our understanding as to why diabetes increases CVD disproportionately across gender, much remains to be learned (96). Additionally, with research lacking on the consequences of BSO in diabetic women, the aim of this present study is to contribute to the understanding of the association between diabetes and cardiovascular disease, and to investigate the moderating role oophorectomy may play on this relationship. Findings from this study will help identify if a causal association exists, and will shed more light on the roles androgens play on the increased risk for CVD in postmenopausal women with diabetes. Furthermore results from this study will provide evidence and add information to the current debate in the scientific community; as to whether the ovaries should be removed or conserved during hysterectomy.

\section{SPECIFIC AIMS OF THE STUDY}

The aims of this study are twofold: first, to investigate whether bilateral salpingooophorectomy (BSO) has a moderating effect on cardiovascular disease risk in diabetic women, including all cause mortality and CVD-related mortality using data from a national survey; and, second to examine, using a large prospective observational study of 
postmenopausal women, the association of baseline levels of testosterone (free and total), sex hormone binding globulins and estradiol with CVD-related mortality in diabetic as well as nondiabetic postmenopausal women, and to determine if BSO influences the association.

\section{HYPOTHESES}

We hypothesize that

1. Postmenopausal diabetic women without ovaries will have lower androgen levels compared to postmenopausal diabetic women with intact ovaries and this difference will significantly influence the risk of CVD.

2. Among postmenopausal women with diabetes, the risk of CVD events will be lower in those who have undergone bilateral oophorectomy compared to those without a history of oophorectomy.

3. If estrogens oppose visceral fat accumulation, then the removal of the ovaries at an early age may have a more adverse effect on cardiovascular health than if this procedure is performed after the time of natural menopause. 


\section{CHAPTER TWO}

\section{BACKGROUND AND SIGNIFICANCE}

\section{Introduction}

The 20th century observed a transition in the burden of disease in industrialized countries as infectious/communicable diseases gave way to chronic diseases especially CVD as the leading cause of morbidity and mortality in the adult population. While improvement in the diagnosis of acute events such as MI increased in the early part of that century, leading to a better understanding of this chronic condition, the underlying causal factors were debated. However a growth in observational epidemiology by the middle of the 20th century brought to light certain lifestyle factors which influence heart disease, as researchers sought to study trends across countries and populations. This work continues today: epidemiologic studies identify traditional and novel risk factors associated with the prevention/control of CVD, while translational and interventional studies seek new and better therapies. Despite substantial progress, CVD continue to affect and negatively impact health and quality of life in men and women universally.

Several epidemiologic studies reveal that clinically relevant differences exist between men and women in terms of incidence, prevalence, management and outcomes of CVD, but less is known about why CVD affects women and men differently (97) 
Historically, heart disease has been perceived as a male disease, a belief that is fueled by the media representation of patients with MI being men and the underrepresentation of women advertisements for cardiac drugs $(98,99)$. This has contributed to fewer women being enrolled in CHD trials. However it is worth mentioning that exclusion and underrepresentation of women in heart disease trials was not necessarily an overt discrimination perpetuated deliberately (98). Rather, the atypical presentation or symptoms of CHD in women made them less likely to be referred to Cardiologist who could, in turn, invite them to enroll in clinical trials. This has resulted in a shortfall of evidence concerning sex-specific outcomes as well as CVD treatment in women (97). While this knowledge gap may explain why cardiovascular health in women is not improving as fast as that of men, gender differences in cultural, behavioral, psychosocial, socioeconomic status and the endocrine- metabolic milieu may be contributory factors (97).

This review outlines the epidemiology of CVD in women with emphasis on gender differences and attempts to provide reasons why these differences exist.

Traditional modifiable risk factors will be briefly described. In recent years there has been an increase in research into the effect of androgens on CVD, against the backdrop of estrogens showing no protective effect on primary prevention of CVD, and an increasing awareness of the connection between the attributes of the Metabolic Syndrome and CVD

In view of this, the review will include a comprehensive discussion on the roles sex-specific characteristics and sex hormones on cardiovascular health. Finally, a detailed discussion of the epidemiology of CVD in diabetic women, and how sex-specific characteristics and sex hormones influence CVD risk in this high risk population. 


\section{EPIDEMIOLOGY OF CARDIOVASCULAR DISEASE}

CVD is the leading cause of morbidity and disability in industrialized countries as well as the leading cause of mortality worldwide. Current estimates from the World Health Organization show that 17.3 million people around the globe die from CVD each year, representing $30 \%$ of all deaths (100). Alarmingly, this figure has been estimated to reach 25 million by the year 2030 (100). Additionally, 7.3 million and 6.2 million deaths that occur annually are attributable to coronary heart disease and stroke respectively (100). Low-and middle-income countries bear the greatest burden of CVD mortality with $80 \%$ percent of all deaths taking place in these countries, where deaths occurs almost equally in men and women (100). In 2004, CVD alone caused approximately $32 \%$ (8.6 million) of deaths in women worldwide compared to $27 \%$ in men (101).

Recent reports from the United States estimate the overall annual rate of death attributable to CVD to be 236.1 per 100,000 (age-standardized rate 237.1 per 100,000) (102), representing $32.3 \%(787,931)$ of the total number of $2,437,163$ deaths documented (102). Furthermore, 2,150 Americans die of CVD each day, which is an average of one

death every 40 seconds (102). Moreover 34\% of all CVD deaths occur in people who are less than 75 years old (102).

Gender differences in the incidence and prognoses (short term and long term) of CVD are well recognized. For instance since 1984, the number of deaths attributable to CVD in American women has exceeded that of men (24). In 2009, $51 \%(401,495)$ of all CVD deaths occurred in women (102). Interestingly, 2 out of 3 women who die suddenly 
of CHD are asymptomatic (103). In addition, women are somewhat more likely to die within one year of suffering a myocardial infarction (MI) compared to men (23\% vs. $18 \%$ ) (104), and nearly half of those women who survive are disabled by heart failure (105). The burden of CVD mortality is also disproportionately distributed by race/ethnicity as Blacks/African American women have a higher rate of CVD mortality (267.9 per 100,000) than Non-Hispanic whites (190.4 per 100,000) (102). Among women aged 20 years and older, the prevalence of CVD is highest among Blacks/African American (48.9\%), followed by Non-Hispanic Whites (32.4\%) and Mexican Americans $(30.7 \%)(102)$

With regards to the prevalence of CVD nationwide, 83.6 million American (1 in 3) are estimated to be living with one or more types of CVD, of whom 42.2 million are aged 60 years and older (102). Of the total number of Americans living with some form of cardiovascular disease, 42.9 million are women (102). The distribution of disease prevalence according to the various CVD are as follows; 15.4 million (6.6 million women) adults have coronary heart disease, 7.6 million (2.6 million women) have myocardial infarction and 6.8 million (3.8 million women) have stroke (102). Before age 75, a higher proportion of CVD events attributable to CHD occur in men while in women, a higher proportion of events is attributable to stroke (102).

Estimates based on data from the Framingham Heart Study (1980 to 2003) indicate that the annual incidence rates for a first CVD event increases with age, with men aged 35 to 44 years having a rate of 3 per 1000 compared to 74 per 1000 in those aged 85 to 94 years (102). Rates are comparable in women; but 10 years later in life, with this gap narrowing with advancing age (102). The older age at onset of CVD in women 
correlates with an increase in comorbid conditions namely hypertension and diabetes, which in turn results in poorer prognoses and consequentially increased mortality (98). Information based on a pooled analysis of five NHLBI funded community based cohorts shows that the lifetime risk of developing CVD (defined as fatal and nonfatal CHD, stroke, congestive heart failure, and all other CVD related deaths) after age 45 is $60.3 \%$ for men and $55.6 \%$ for women (106).

Despite these alarming statistics and the increased awareness over the past decade, CVD is still viewed by many women as a remote health risk. In 2009, a survey conducted by the American Heart Association reported that almost half (54\%) of women (which is an improvement over the $30 \%$ reported in 1997) recognized heart disease as a significant cause of mortality in women (107). Alarmingly only 16\% in 2009 (7\% in 1997) identified heart disease as the single greatest health problem facing women compared to $28 \%$ who were most concerned about breast cancer $(107,108)$.

\section{EXPLAINING THE GENDER DISPARITY}

Reasons for the observed gender disparity are no doubt multifactorial, but may be explained in part by the following. First, there are difficulties in the diagnosis of CHD in women. Results from several observational studies confirm that the accuracy of diagnostic tests differs between men and women (31). For instance, the standard exercise stress test which is the most commonly used method for diagnosing coronary artery disease (CAD) has lower sensitivity and specificity in women compared to men (31, 109). Menopausal status, hormone therapy use and a lower prevalence of obstructive 
CAD in women may be contributory factors (31). In addition, women undergoing exercise stress tests tend to have lower functional capacity which limits the induction of myocardial ischemia and in turn decreases the diagnostic ability of the standard exercise stress test to detect CAD (28). All these factors also limit the use of stress echocardiogram in women, necessitating the use of non-imaging tests to detect vascular obstruction $(28,30,109)$. Furthermore, complimenting standard exercise stress testing with other specialized diagnostic instruments such as myocardial perfusion Single Photon Emission Computed Tomography (SPECT) or dobutamine stress echocardiography, which all have relatively higher specificity, may aid in better diagnosis and prediction of future adverse events in women, but at an added expense $(28,30)$.

Second, women may not experience the typical symptoms of angina. Rather, their pain may be located in the right arm associated with fatigue, abdominal pain, nausea, sweating and shortness of breath $(27,30,110)$. Women who experience signs and symptoms of angina and MI tend to delay seeking medical care, and when they do may not be evaluated as aggressively as men, or may be misdiagnosed $(27,30)$. Women respond to CHD treatment drugs differently than men, and have more adverse drug reaction $(29,30)$. This is further compounded by the hindering of inclusion, if not exclusion, of women from clinical drug trials. For example, of the over 30,000 participants enrolled in randomized trials to study the effectiveness of statins in primary and secondary prevention of CVD, only $10 \%$ and $23 \%$, respectively, were women (98). Moreover, between 1997 to 2006, 27\% (range:10\% to 47\%) of the total enrollment in phase 3 or 4 cardiovascular disease randomized clinical trials funded by the National Heart, Lung, and Blood Institute were women (NHLBI) (111). The underrepresentation 
of women in clinical drug trials impedes determination of the benefits of drug therapy for women because of low statistical power $(28,31)$. Therefore clinicians cannot be certain whether results with new drugs apply to women, since drug absorption and metabolism may differ in women and men due to lower body weight, higher body fat and differences in circulating hormone levels $(30,31,98)$.

Third, women who undergo percutaneous coronary interventions and CABG have more fatal complications related with these procedures than do men (27). For example, women are twice as likely to die during surgery from CABG, and are more likely to undergo repeated revascularization procedures (112). On average, women undergoing coronary revascularizations are older and tend to have higher burden of comorbidities (27). It's now recognized that women have smaller coronary arteries independent of body size $(113,114)$. Furthermore, compared to men, women with established CHD, have coronary vasculatures characterized by smaller luminal diameter, stiffer vessels as a result of fibrosis or positive remodeling, and contain more diffuse atherosclerosis with the tendency for plaque erosion as opposed to rupture $(31,115-117)$. These factors all lead a greater susceptibility for complications during revascularization procedures compared to men (27).

Recognition of the gender disparity in clinical trials enrollment, and a growing awareness in the scientific community, as well as women's advocacy groups, of the differences in pathophysiology, presentation, treatment and outcomes of CVD in women, led to the enactment of a series of federal mandates aimed at increasing enrollment by women in CVD trials to bridge the knowledge and disparity gap (118). This led to the creation in 1990 of the Office of Research on Women's Health by the U.S Congress to 
ensure fair representation of women in CVD clinical trials (118). Backed by the NIH Revitalization act (Public law 103-43), large single sex trials sponsored by the NHLBInamely the Women's Health Study and the Women's Health Initiative--were commenced, enrolling thousands of women in the study of primary prevention of heart diseases (118).

Over the past 30 years, the enrollment of women (42.6\% in primary and $26.6 \%$ in secondary prevention) in all cardiovascular trials, either government/foundation-funded or industry-funded has increased worldwide (119). In 1970 only 9\% of all trials included women but this increased to $41 \%$ in 2006 . Nevertheless, among clinical trials conducted in the United States, lower estimates were reported with women making up only $26.7 \%$ (119). Also no significant increase in enrollment of women in mixed sex trials was seen over this period. Improvement was seen in NHLBI-sponsored mixed-sex CVD clinical trials, with women forming $38 \%$ and $26 \%$ of CAD trials and congestive heart failure (CHF) trial participants respectively (118). When these large multicenter single sex trials are included, however, the estimates increase to $57 \%$ for all CAD trials but estimates remains the same for studies of CHF (118).

While these figures and statistics are encouraging, improvement is needed to ensure that trials generate the information necessary for evidence-based medicine involving women, to aid clinicians in making informed decisions, to reduce or avoid the negative side effects of therapy and to tailor treatment to this patient group to achieve the desired outcomes in clinical care. Additionally, a gender-sensitive approach to research should be incorporated, with more women enrolled in drug and intervention trials as well as increasing the education for women about the symptoms of heart diseases. Lastly, increased research is needed that focused on women with comorbidities such as diabetes, 
which has been demonstrated to be a stronger risk factor for CVD in women compared to men. A better understanding of the effect of such comorbidities in women, especially during menopause, will help elucidate why such women are at high risk for CVD disability and mortality compared to men.

\section{THE NEED FOR PREVENTION}

Aside from morbidity and mortality, CVD has a tremendous impact on the economy of nations. CVD costs the United States $\$ 312.6$ billion each year (192.1 billion due to direct costs of expenditures and $\$ 120.5$ billion due to indirect costs of lost productivity), compared to the estimated cost $\$ 228$ billion for all cancer care (102).

Although enormous, this figure has been forecasted to increase to $\$ 818$ billion by the year 2030, primarily due to population aging and the detrimental effects of diabetes and obesity (120). During the period 2000 to 2004, CVD accounted for approximately $17 \%$ of all national health expenditures and nearly 30\% of all Medicare expenditures (121). These figures stress the importance of improving cardiovascular health and quality of life through risk factor modifications, particularly among women since they have longer life expectancy and are more likely develop and dye from CVD.

Although an increasing number of women (48\%) are proactively discussing heart disease risk with their primary care physicians (107), unfortunately, preventive therapies are less often recommended to women (66). This is largely explained by lower perceived risk of CVD among women by healthcare providers leading to under-identification and under-treatment of modifiable risk factors (66). For instance, in a national survey of 500 
randomly selected physicians (300 primary care physicians, 100

obstetricians/gynecologists, and 100 cardiologists) to identify awareness and adherence to cardiovascular disease prevention guidelines, only $20 \%$ reported knowing that more women than men die each year from CVD (122). Also, primary care physicians were more likely to assign intermediate risk women—as assessed by the Framingham risk score - to a lower risk category than men with identical risk profiles, while reassigning men with intermediate risk to a higher risk category (122).

The need for early identification of women at high risk for CVD and implementation of appropriate lifestyle modifications and treatment are immense public health issues of importance. Currently, the commonly used risk prediction algorithm to identify women at high risk for CHD is the Framingham risk score (123). However this risk estimation method does not include reproductive factors that increase a women's risk for a CHD event, and therefore may not be entirely appropriate for women. Although the Reynolds score system does not include reproductive risk factors, it has been recommended by some researchers to be used in women for risk estimation since it reclassified 40 to $50 \%$ of women enrolled in the Women's Health Study at intermediate risk into higher or lower risk categories (124). While this risk algorithm holds promise, it needs to be validated in other populations (123).

\section{RISK FACTORS}

One of the largest studies of MI risk factors in women, the INTERHEART study, a global case control study which included participants from 52 countries reported that 
nine traditional risk factors collectively explained more than $90 \%$ of acute MI in both men and women across all major ethnic groups (125). These nine modifiable risk factors are dyslipidemia, psychosocial stress, hypertension, smoking, diabetes, physical inactivity, obesity (especially abdominal), poor diet (with insufficient fruit and vegetable intake), and excessive alcohol consumption. These risk factors however impacted acute MI risk differentially with regard to gender. Among women, the population attributable risk of psychosocial stress, hypertension, diabetes and physical inactivity were greater compared to men (125). While the direct effects of age and family history of heart disease were not addressed in this study, they are important non modifiable risk factor for CVD in both men and women. Certain subgroups of women are known to have a significantly higher risk of CVD which cannot be entirely explained by these aforementioned traditional risk factors. These include women with systemic autoimmune diseases namely systemic lupus erythematosus and rheumatoid arthritis (126). Other sex specific factors such as life-cycle changes (puberty, menstrual cycle, polycystic ovary syndrome, pregnancy, and menopause) and sex hormone (estrogens and androgens) are suggested to impact the incidence of CVD; howbeit the possible biological or genetic mechanisms underlying their effect are not well understood (31).

Aside from these traditional CVD risk factors, other novel risk markers have been identified. In an effort to improve risk prediction, over 100 risk markers (i.e. C-reactive protein, lipoprotein (a), homocysteine, etc) have been proposed to predict CVD particularly in intermediate-risk populations as assessed by existing risk algorithms. However these biomarkers are not accepted for populations screening by consensus conferences since they are either not causally related to CVD, or statistical and 
epidemiological evidence indicate that they add little to traditional risk factors in risk estimation $(123,127)$.

The following paragraphs describe some of the important modifiable risk factors and their effects on atherosclerosis progression and CVD among women. Gender differences that may exist will be highlighted.

\section{Traditional Risk Factors}

\section{Smoking}

Smoking is the single most important preventable cause of heart disease in women and the leading cause of heart disease in women less than 50 years old (123). Cigarette smoking was uncommon among women until the 1920's (128). Since 1965, when $33 \%$ of women ( $51 \%$ of men) were smokers, the rate has significantly decreased, although the rate of decline has been lower for women (128). One reason for this reduced decline is that women may smoke cigarettes for weight control (66). As of 2009, 16.7\% (19.6 million) of women aged 18 years and older were reported to be current smokers (102). A dose response relationship exists between the number of cigarettes smoked per day and the incidence of non fatal CHD. Smoking 1 to 4 cigarettes daily raises the risk of CHD in women by as much as threefold, while smoking 25 or more cigarette increased the risk six fold (129). Additionally smoking has a more harmful effect on women than men, with women smokers having almost $60 \%$ increased risk of first admission to a hospital or death caused by MI (Hazard Ratio: 1.57, 95\% Confidence Interval: 1.25-1.97) compared to men (130). Furthermore, women who smoke and use oral contraceptives have a twofold risk of MI (Odds Ratio: 2.0, 95\%CI: 1.5 to 2.8 ) mainly due to venous 
thrombosis (131). Exposure to passive smoking also raises the risk of CHD by $24 \%$ (22\% in men) (Relative Risk, 1.24; 95\%CI: 1.15-1.34) (132). Regardless of age or gender, smoking cessation reduces heart disease risk, with one year of cessation resulting in a reduction of approximately 50\% (128). After 10 years of smoking cessation, the risk of CHD approaches that of never smokers (128). Smoking influences heart disease risk in women through several mechanisms. These include endothelial dysfunction, increased LDL oxidation, vascular inflammation, platelet aggregation, reducing levels of endogenous estrogens and causing menopause to occur at an earlier age $(66,133)$,

\section{Hypertension}

Numerous epidemiologic and interventional studies have long shown that hypertension is associated with CVD, especially CAD, stroke and heart failure. Hypertension and stroke cause the greatest morbidity in women (66). In adults, hypertension is defined as a systolic and/or a diastolic blood pressure measurement which is consistently above $140 / 90 \mathrm{mmHg}(134)$. The prevalence of hypertension is low in premenopausal women but increases with age. The lifetime risk of hypertension for women after age 55 is more than 90\% (66). Currently 1 in 3 women (40.7 million) aged 20 years and older is hypertensive, with those aged 75 years and older bearing the greatest burden of disease $(80.1 \%)$ (102). While hypertension was associated with a threefold increase in CHD risk in men aged 65 to 74 in the Framingham Heart Study, a fourfold increased risk was observed among women of the same age (112). In the INTERHEART study of acute MI, the population attributable risk for hypertension was 
$36 \%$ in women compared to $19 \%$ in men, underscoring a greater impact of high blood pressure in women (125).

There are several pathophysiologic mechanisms by which hypertension influences cardiovascular heath. High blood pressure induces endothelial dysfunction, remodeling of coronary arteries and increased resistance at the microvascular level (135). All these processes promote and exacerbate the atherosclerotic process by making plaques formed in the arteries more unstable (135). Unstable atherosclerotic plaques are prone to rupture which exposes thrombogenic material, such as collagen to the circulation resulting in thrombus formation in the lumen of coronary arteries (136). These intraluminal thrombi either occlude coronary arteries resulting in infarctions, or more often detach and occlude other smaller vessels causing thromboembolisms (136). An ischemic stroke occurs when thrombi occlude the carotid artery (136). Additionally, hypertension leads to left ventricular hypertrophy which promotes a decrease of coronary reserve while increasing myocardial oxygen demand, which collectively contributes to the incidence of MI by the action of ischemia (135). Additionally, hypertension is frequently associated with other metabolic disorders such as dyslipidemia and hyperinsulinemia which are all risk factors for CVD (135).

In pregnant women, hypertension is the most common medical problem encountered, accounting for $15 \%$ of all complications (137). This complication includes a spectrum of conditions including preeclampsia-eclampsia, preeclampsia superimposed on chronic hypertension, chronic hypertension and gestational hypertension (138). Compared to normotensive women, hypertensive women have an increased risk of incident preeclampsia during pregnancy as well as increased complications resulting from 
this condition (25\% vs. 6\%) (137). Preeclampsia which most commonly occurs during the first pregnancy is defined as the development of hypertension and proteinuria after 20 weeks of gestation in previously normotensive persons. (137), When it occurs in women with preexisting hypertension, its termed chronic or superimposed preeclampsia (137). In industrialized countries preeclampsia affect $2-8 \%$ of first pregnancies and results in about $15 \%$ of maternal mortality globally (139). Aside pregnancy complications, preeclampsia further increase the risk of CVD events (66). At the turn of the century, a Norwegian which assessed the effect of preeclampsia on CVD risk reported that among women with preeclampsia and who also had a premature delivery ( $<36$ weeks), the risk of CVD mortality was a staggeringly eightfold compared to women who had a normal delivery without preeclampsia. Furthermore, a Meta analysis of 25 prospective and retrospective cohort studies including 198,252 women with preeclampsia showed that a history of this condition increased the risk of incident ischemic heart disease (RR: 2.16, 95\% CI:1.862.52) after 11.7 years, stroke (RR: $1.81,95 \%$ CI:1.45-2.27) after 10.4 years, and venous thromboembolism (RR:1.79, CI:1.37- 2.33) after 4.7 years (140). Preeclampsia amplifies the effect of hypertension on cardiovascular health by increasing free radical derived oxidative stress and various inflammatory markers which results in severe endothelial dysfunction and subclinical vascular damage (138).

\section{Dyslipidemia}

An estimated 53.6 million (44.9\%) American women aged 20 years and older have total serum cholesterol levels greater than or equal to $200 \mathrm{mg} / \mathrm{dL}$, a figure higher than the $41.3 \%$ (45.3 million) reported for men (102). Furthermore, 30.0\% (31.9\% in 
men) of women are reported to have low-density lipoprotein (LDL) Cholesterol levels greater than or equal to $130 \mathrm{mg} / \mathrm{dL}$, as well as $12.3 \%$ (31.8\% in men) having less than 40 $\mathrm{mg} / \mathrm{dL}$ of high-density lipoprotein (HDL) Cholesterol (102).

Dyslipidemia is a significant independent risk factor for CVD in both women and men, however there are considerable differences in lipoprotein particle with regards to gender exists (66). Women generally have higher levels of total cholesterol, secondary to more HDL cholesterol throughout their lifespan (66). Beginning at puberty, women have consistently higher HDL levels than men although during the menopausal transition, a slight reduction (about 10\%) is observed (66). Conversely, LDL and very-low-density lipoprotein (VLDL) cholesterol tend to be lower in women compared to men until about age 60, when levels begin to rise and surpass those of men (66). Triglycerides, on the other hand, increases with age in both genders, howbeit at a slower rate in women, but after age 79 , women have similar levels to men (66).

While higher levels of HDL are associated with a lower CVD risk and other comorbid conditions including hypertension, obesity and diabetes, the opposite is observed with triglycerides, LDL and total cholesterol (66). Hypertriglyceridemia and low HDL cholesterol levels are more important risk factors for women than men (112). A meta-analysis of 17 prospective studies which included 46,413 men and 10,864 women found that with each $1 \mathrm{mmol} / \mathrm{l}$ increase in fasting triglyceride levels, there is a corresponding $30 \%$ increased risk of CVD in men compared to a $76 \%$ increase risk in women. Further adjustment for HDL cholesterol levels attenuated the risk but remained significant, with women (RR: 1.37, 95\%Cl: 1.13-1.66) still having an increased risk compared to men (RR: 1.14, 95\%CI: 1.05-1.28) (141). The Framingham 
Heart Study as well as the Lipid Research Clinics Prevalence Mortality Follow-up Study published similar results showing that each $0.025 \mathrm{mmol} / \mathrm{L}$ increase in $\mathrm{HDL}$ cholesterol levels reduced CVD events to a greater extent in women than men (97). In the former study, a 3\% (2\% in men) decrease in the incidence of CAD in women was observed (142), while the latter study reported a 4.7\% (3.7\% in men) reduction in CVD mortality among women (143).

Reduction in cholesterol levels (excluding HDL) by lipid-lowering therapies has been shown in several intervention trials to substantially influence cardiovascular health (133). Although some have found comparable effectiveness in both genders, a few studies reveal greater effectiveness in women. One of those studies is the cholesterol and recurrent events (CARE) trial which assessed the effect of Pravastatin in the secondary prevention of CHD (144). After five years of follow-up, postmenopausal women in the treatment arm had a reduced risk of $43 \%$ for coronary events (which was defined as coronary death, nonfatal MI, PTCA or CABG) and 56\% for stroke (144). Although men showed a similar reduction in risk, the magnitude was less than that reported for post-MI women with average cholesterol levels. Data from another meta-analysis of 27 randomized trials which included approximately $26 \%$ of women to assess the effect of statins on CVD in persons with low risk of vascular events, showed a $21 \%$ reduction in non-fatal myocardial infarction or coronary death (RR: $0 \cdot 79,95 \%$ CI: $0 \cdot 77-0 \cdot 81$ ) with each $39 \mathrm{mg} / \mathrm{dl}$ reduction in LDL level (145). This significant finding was independent of age, sex, baseline LDL cholesterol or previous vascular disease. However in participants without a history of any vascular disease, the risk of non-fatal myocardial infarction or coronary death and all-cause mortality was statistically significantly reduced by $15 \%$ and 
9\% respectively, with each $39 \mathrm{mg} / \mathrm{dl}$ reduction in LDL cholesterol (145). The Justification for Use of statins in Prevention: an Intervention Trial Evaluating Rosuvastatin (JUPITER) trial which enrolled 17,802 participants (38\% women) also confirmed the effectiveness of statins in the primary prevention of CVD in women. After a median follow-up of 1.9 years, rosuvastatin use (20 mg daily) was associated with a $54 \%$ reduction in MI, $48 \%$ reduction in stroke, $46 \%$ reduction in revascularization, $43 \%$ reduction in venous thromboembolism, and $20 \%$ reduction in total mortality (146). This reduction in risk was similar among men and women, but overall women had a greater reduction ( $46 \%$ vs. $42 \%$ ) in the composite endpoint of nonfatal MI, nonfatal stroke, hospitalization for unstable angina, arterial revascularization, or cardiovascular mortality (146). Recently, another meta-analysis of all primary prevention statin trials in women with 13,154 participant (mean age of 63 to 69 years), also found a significant reduction in CVD events with statins by a third (RR: 0.63, 95\%CI: 0.49-0.82) (147). While such results lend support to the conclusions of the JUPITER trial that statin therapy is effective in the primary prevention of CVD in women, it's uncertain if the same effect will be found in younger women since most primary prevention trials included only older postmenopausal women

Despite the years of research on cholesterol and CVD risk, the underlying mechanisms are still not entirely understood (96). LDL cholesterol when high accumulates in the arterial walls and is oxidized, and taken up by foam cells, initiating as well as progressing atherosclerotic process (148). HDL cholesterol on the other hand directly opposes atherosclerosis by removing cholesterol from foam cells, inhibiting LDL oxidation as well as limiting the inflammatory processes that underlie atherosclerosis 
(148). Furthermore HDL has antithrombotic properties that prevent the formation of blood clots in the arteries preventing vascular occlusions (148). The action of statins on cardiovascular health is achieved via its effects on lowering arterial inflammation and inhibiting cerebral cholesterol synthesis (96). Aside from cholesterol lowering, statins also exert pleiotropic properties such as increasing endothelial nitric oxide formation as well as suppressing vascular inflammation which inhibits atherosclerosis (149).

\section{Obesity, Diet and Physical inactivity}

Obesity is a growing public health problem worldwide and has been shown to increase CVD risk as well as exacerbates numerous other chronic diseases (102). In epidemiologic studies, body mass index (BMI) is used to measure general obesity, but this index does not account for variation in body fat distribution and abdominal fat mass. To capture central or abdominal adiposity, other anthropometric measures such as waist circumference (WC) and waist-to-hip ratio (WHR) have been recommended (150). A BMI $\geq 30 \mathrm{~kg} / \mathrm{m}^{2}$ defines obesity with values of $25.0-29.9 \mathrm{~kg} / \mathrm{m}^{2}$ define overweight (150). Unlike BMI which uses the same categorization for both men and women, WC and WHR have different cutoff values for abdominal obesity. In women $W C$ of $\geq 88.0 \mathrm{~cm}$ and WHR of $\geq 0.80$ defines central obesity (150). In the United States, $64 \%$ of women are reported to have a BMI $\geq 25 \mathrm{~kg} / \mathrm{m}^{2}$, while $36 \%$ are obese (102). Blacks/African American women are more likely to be obese (54\%), compared to Mexican-Americans (45\%) and non-Hispanic whites (33\%) (102). Forecast, using the Behavioral Risk Factor Surveillance System (BRFSS) estimates that by 2030, 51\% of the population will be obese, with $11 \%$ having severe obesity (102). This database further shows that the 
number of quality-adjusted life-years lost as a result of obesity is similar to or greater than those lost as a result of smoking (102)

Several studies reveal that obesity increases the risk of CVD. In the a metaanalysis of 58 cohorts, with 221,934 participants and 14,297 first-onset CVD events, a minimal increased risk of $7 \%, 10 \%$ and $12 \%$ using BMI, WC and WHR respectively in CVD was reported (151).An earlier meta-analysis (152), however reported a greater risk of CHD event in both overweight men and women. Summarizing information from 302,296 participants with 18,000 events, the authors reported an $18 \%$ and a $50 \%$ increased risk for CHD among overweight and obese individuals (152). In contrast, the Framingham Heart Study reported that obesity infers a greater effect on cardiovascular health in women than men, with obese women having a 64\% (46\% in men) increased risk of first CVD as well 2.75 fold (2.21 in men) risk for hypertension (153). Despite these figures illustrating that obesity increases CVD, an obesity paradox exists with regards to certain cardiac diseases (154). Among patients with a history of various types of CVD, those who are overweight or obese have been reported to have better outcome than their normal weight counterparts. This paradox has been reported for outcomes including heart failure (155), peripheral arterial disease (156), acute coronary syndrome (157) and cardiovascular mortality (158).

An increase in caloric intake and physical inactivity is closely tied to the current obesity epidemic. A well balanced diet with adequate fruits and vegetables coupled with increased physical activity has been shown to aid in weight management and to reduce the risk of CVD in the long run (128). A single-blind randomized trial of 130 severely obese (class II or III) adults without diabetes, followed for 12 months reported that both 
intensive lifestyle intervention of diet and physical activity achieve clinically significant weight loss and improvement cardiometabolic factors namely waist circumference, visceral abdominal fat, hepatic fat content, blood pressure and insulin resistance (102, 159). Moreover, physical activity promotes greater reductions in waist circumference and hepatic fat content (159). These reported benefits of diet and physical activity are less marked though in women compared to men (133).

The Nurses' Health Study (160) and the Women's Health Initiative (161) both showed a strong, graded inverse association between physical activity and the risk of coronary events. Additionally, they report that brisk walking showed similar effect on cardiovascular health as vigorous exercise; reducing CVD risk by as much as 40\% (160). In spite of these benefits from physical activity, $33.2 \%$ of women ( $29.9 \%$ in men) report no physical activity, with minority populations (Blacks: $41.1 \%$ and Hispanics: $42.2 \%$ ) having higher age adjusted estimates compared to non-Hispanic whites (27.7\%) (102).

Obesity increases the risk of CVD through several mechanisms. For instance adipose tissue produces cytokines such as leptin and adiponectin, while increasing levels of inflammatory markers and endothelial cell products namely intercellular adhesion molecule-1 (162). These bioactive mediators affect body weight homeostasis and predispose to insulin resistance (162). Additional effects of these bioactive mediators are alteration in lipid profile and blood pressure as well as increased oxidative stress, coagulation, and fibrinolysis which all lead to endothelial dysfunction and atherosclerosis (162). Furthermore, an obesity induced insulin resistance state is characterized by increased levels of non-esterified fatty acids which promote lipotoxicity and in the long run initiates atherosclerosis (162). 


\section{CARDIOVASCULAR DISEASE IN DIABETIC WOMEN}

\section{Epidemiology of diabetes}

About 23.6 million (7.8\%) Americans have diabetes mellitus with 5.7 million of this number being undiagnosed (3). Among the various ethnic/racial groups, $12.6 \%$ of non-Hispanic blacks, $11.8 \%$ of Hispanics, $8.4 \%$ of Asian Americans and 7.1\% of nonHispanic whites aged 20 and over are reported to be living with diabetes (102). Each year, about 1.9 million new cases of diabetes are diagnosed in US adults (102). Diabetes is the seventh leading causes of death with rates of 23.3 per 100,000 for white males, 44.2 per 100,000 for black males, 15.7 per 100,000 for white females and 35.9 per 100,000 for black females (102). Furthermore $68 \%$ and $16 \%$ of deaths in persons diagnosed with diabetes are due to heart diseases and stroke respectively (102). Moreover, diabetes is associated with a cluster of CVD risk factors. For instance the prevalence of hypertension, elevated LDL cholesterol and obesity in diabetics is $75 \%$ to $85 \%, 70 \%$ to $80 \%$, and $60 \%$ to $70 \%$ respectively (102). Results from the Framingham Heart study show that the lifetime risk for CVD among women with diabetes is $54.8 \%$ among normal-weight women (78.6\% in normal-weight men) and $78.8 \%$ among obese women (86.9\% in obese men) (163). The annual total cost (direct and indirect) of diabetes care grew to $\$ 245$ billion in 2012 from $\$ 174$ billion in 2007, with the medical care cost per each diabetic person estimated to be $\$ 7,900$ (164). With the increasing obesity epidemic and aging of the population, diabetes will undoubtedly place a great burden on the future healthcare system. 


\section{The Impact of Cardiovascular Disease in Diabetic women}

One of the most important risk factors for heart disease in women is type-2 diabetes. Compared to persons without diabetes, the risk of CVD among diabetics is about 3-fold (102). Among diabetics aged 65 and older, heart disease accounts for $68 \%$ of all deaths (3), and diabetes is a stronger risk factor for CHD in women than men (10). The risk of fatal and non fatal CHD events for diabetic women is reported to be as high as 9.5 fold compared to 2.8 fold for diabetic men (9). Furthermore, women with diabetes are more likely to die of CHD compared to non diabetic women or men with and without diabetes (5). A twenty year follow-up of the Nurses' Health Study cohort reported an 18 fold risk for CHD mortality among women with both a history of diabetes and CHD, compared to an 8 fold increase risk among women with a history of CHD as well as those with a history of diabetes (10). Additionally, diabetes has more debilitating effects in women than men (6-9). Inflammatory factors due to diabetes in women are proposed to interact with female hormones, resulting in a decreased effect of estrogen on the coronary vasculature, insulin actions and body fat distribution thereby abolishing the gender gap in heart disease rate among premenopausal women and men of similar age (9). The presence of diabetes in postmenopausal women increases the progression of atherosclerosis as well as the incidence of heart disease (165).

With recent progress in reducing the prevalence of many CVD risk factors such as smoking, hypercholesterolemia, etc, a reduction in all-cause and CVD-related mortality has been observed among diabetics. During the period of 1997 to 2006, the CVD death rate declined by $40 \%$, while all-cause mortality declined by $23 \%$ among diabetics (166). 
However, there was a greater magnitude of decline among men than women (166). While the decline in mortality among diabetics is encouraging, the increasing incidence of diabetes will result in a greater burden of disease in the future. The lack of improvement in survival among diabetic women compared to men is disturbing, and progress seems unlikely until the reasons for this gender difference are determined. While some have attributed this to the lesser improvement in CVD risk factors, others point to a less aggressive medical management and worse outcomes after coronary revascularization in diabetic women (167).

The treatment of diabetes and CVD is complex and certain factors including disease status, comorbidities, self-management capabilities and patient's compliance are all considered before therapy is prescribed (168). Although diabetes treatment is intensified if risk factors for CVD or CVD itself is present, gender differences in diabetes care in the presence of heart disease has been reported. The DIANA study found that diabetic women are less likely to be prescribed angiotensin-converting-enzyme (ACE) inhibitors and calcium channel blockers compared to men with CHD (168). These gender disparities exist even after controlling for confounding factors such as age, diabetes duration, glycosylated hemoglobin (HbA1c) level, medication adherence, and the prevalence of CVD, diabetic nephropathy and depression (168). Similar studies also report that among diabetics who are eligible for statin therapy based on recommendations by American Diabetes Association, women are less likely to receive adequate lipid lowering treatments compared to men (169). Some researchers have argued that this disparity in treatment which favors men is not necessarily intentional but that the control 
of hyperglycemia and major CVD risk factors are less satisfactory in women than men (170).

Although women undergoing coronary intervention in general have a higher frequency of adverse outcomes than men, those with diabetes have increasingly worse outcomes primarily due to diffuse small-vessel disease leading frequently to diabetic cardiomyopathy (171), A register linkage analysis of outcomes among diabetic patients with acute MI who have undergone coronary interventions (171) reports that after 2 years of follow-up (which began on the date of PCI during the index hospitalization) the incidence of adverse outcomes was significantly higher in women. Diabetic women were $44 \%$ more likely to encounter a non-fatal acute MI, repeat revascularizations and allcause mortality than men. This excess risk was largely driven by a high (12.0\% vs. 3.9\%) mortality during hospitalization of diabetic women. These results do not only warrant special attention in treatment strategies of diabetic women, especially during hospitalization (171), but also stress the importance of preventing diabetes as well as CVD in women to improve their quality of life.

\section{PATHOPHYSIOLOGY OF DIABETES AND CARDIOVASCULAR DISEASE}

Diabetes is a common endocrine disorder. There are two major types. Type-1 diabetes, known previously as Juvenile-diabetes, is an autoimmune disorder which occurs when the pancreatic beta cells (in the islets of Langerhans) are destroyed thereby causing severe insulin deficiency. Also known as juvenile-onset diabetes since its common in children, there are no means of prevention. Type- 1 diabetes accounts for at least $10 \%$ of 
all cases, and prevalence differs markedly by racial group with Non-Hispanic white having the highest rate of disease (172).

On the other hand, Type 2 diabetes accounts for approximately $90 \%$ of all diabetes nationwide (173). Unlike type 1 diabetes which occurs as a result of absolute insulin deficiency, type 2 diabetes is characterized by insulin sensitivity or peripheral insulin resistance, impairments in the regulation of hepatic glucose production as well as pancreatic beta cell dysfunction (173). Formerly considered as an adulthood disease, type 2 diabetes accounts for $15 \%$ of all cases of diabetes in children between the ages of 10 to 19 years, however the prevalence of diabetes in the children aged 10 to 19 years in the general population is very low $(0.4 \%)(173)$. Although the specific mechanisms underlying the development of type 2 diabetes are complex not well understood, the familial occurrence suggests genetic predispositions which are influenced by environmental factors to result in the onset of clinical disease (173). Some environmental factors that place increased secretory burden on the pancreatic beta cells include puberty, pregnancy, sedentary lifestyle and obesity (173), while acquired risk factors for insulin insensitivity include advancing age, overweight, increased abdominal fat, hypertension, lack of physical activity, decreased HDL cholesterol and medication (173, 174). For brevity "diabetes" will denote type 2 diabetes-which is the focus of this review.

Insulin resistance often always occurs before the onset of diabetes and indicates the presence of an impaired biologic response to either exogenously administered or endogenously secreted insulin (173). It is manifested by decreased insulin-stimulated glucose transport and metabolism in adipocytes and skeletal muscle as well as impaired suppression of hepatic glucose output (173). Hyperglycemia occurs because of 
inadequate utilization by insulin target tissues and also elevated gluconeogenesis. The mechanisms controlling the interplay of these two impairments: hyperglycemia and insulin resistance, are unclear.

\section{Genetic factors involved in the pathogenesis of diabetes}

Clearly, the development of diabetes is associated with a family history of the disease. While concordance rates between monozygous twins for type 1 diabetes is reported to be approximately $50 \%$ or less (175), those for type 2 diabetes is about $90 \%$ (176). Genetic mutations or abnormalities in glucokinase genes, mitochondrial genes, and insulin receptor genes have been observed to influence several processes including the regulatory system of glucose metabolism and insulin action, as well as glucose-stimulated insulin secretion of pancreatic beta cells, which independently lead to the development of diabetes (177). Current evidence show that these genetic abnormalities collectively explain about $30 \%$ of the genetic factors for diabetes (177).

Genetically, diabetes consists of monogenic and polygenic forms, with the genes involved in the common polygenic forms of diabetes being far more difficult to identify and characterize compared to those for monogenic forms (173). Unlike the monogenic forms of diabetes in which environmental factors do not greatly influence the risk of a genetically predisposed person developing clinical diabetes (but genes being the main cause), the polygenic form has both environmental and genetic factors equally influencing the incidence of disease (173).

The mongenic forms are relatively rare and account for only a small percentage of all diabetes cases, however, recognizing these forms are of tremendous importance since 
it will aid in the determination of the best choice of therapeutic agents as well as enhance genetic counseling (178). They are usually diagnosed in the first 2 to 3 decades of life, however if only mild, asymptomatic elevations in blood glucose occur, their diagnosis may be missed until later in life (173).

In monogenic forms associated with insulin resistance, mutations in insulin receptor gene occurs which impair their function, resulting in a decrease in the number of receptors expressed on cell surfaces, accelerating the rate of receptor degradation or inhibiting the transport of receptors to the plasma membrane (173). Insulin resistance associated with insulin receptor mutations manifest during the neonatal period as leprechaunism and Rabson-Mendenhall syndrome, while in adulthood they result in diabetes with marked hyperinsulinemia, acanthosis nigricans, and hyperandrogenism which collectively are referred to as type A insulin resistance (173). Furthermore, mutations in the transcription factor Peroxisome Proliferator-Activated Receptor- $\gamma$ (PPAR $\gamma$ ) also results in diabetes of early onset (familial lipodystrophy type 3) (173). Finally, insulin resistance which is associated with lipoatrophy, lipodystrophy and characterized by a paucity of fat and hypertriglyceridemia results in lipoatrophic diabetes, another monogenic form of diabetes (173).

With regards to insulin deficiency, several mutations occur which result in defective insulin production. These include mutations in insulin or proinsulin genes, and mitochondrial gene which leads to diabetes (173). Maturity-onset diabetes of the young (MODY) which is also a monogenic form of diabetes with an autosomal dominant mode of inheritance is a genetically heterogeneous group of disorders characterized by 
nonketotic diabetes mellitus $(173,178)$. It is common in children and adolescents with onset usually before age 25 years $(173,178)$.

The interactions of several genetic and environmental factors through complex mechanisms result in common polygenic form of diabetes in predisposed persons. The phenotypic manifestations of polygenic form of diabetes include resistance to the action of insulin in muscle, fat, and liver; defects in insulin secretory responses from the pancreatic beta cell (173).

\section{Roles of environmental factors}

As mentioned earlier, aging, obesity, alcohol intake, smoking, and sedentary lifestyles are all independent risk factors for diabetes (177). Since majority of diabetics have central obesity, abdominal obesity is thought to play a crucial role in the pathogenesis of diabetes. Additionally, the mechanism for the cause in reduction in insulin secretion -which is progressive-is not fully known, and may not also be a gradual consequence of insulin resistance since not all insulin resistance subjects develop diabetes. However impaired insulin secretion by pancreatic beta cells generally involves glucose toxicity and lipo-toxicity (177). The impairment of insulin action in major target organs such as liver and muscles to sensitize the absorption of glucose by cells due to either a defect in cell receptors or an absorption effect results in a state termed insulin resistance $(172,177)$. Investigations into the molecular mechanism for insulin action has clarified how insulin resistance is associated to genetic and environmental factors namely hyperglycemia, free fatty acids, inflammatory mechanism, etc (177). 
Abdominal adipose store especially visceral fat, is known to be resistant to the antilipolytic effects of insulin, including alterations in lipoprotein lipase activity which results in increased lipase activity and a greater flux of fatty acids into the portal circulation and to the liver (173). This increased influx of free fatty acids to the liver promotes gluconeogenesis and hepatic insulin resistance, and lead to an accelerated synthesis of very-low-density lipoprotein and increased triglyceride levels (179). To maintain metabolic homeostasis, excess nutrient intake (carbohydrate, protein, or lipid) are stored as fatty acids in the form of triglycerides in adipose tissues(173). If the storage capacity of adipose tissue is exceeded, triglycerides are usually diverted from adipose tissues and stored in non-adipose tissues such as hepatocytes, vascular cells, beta cells and skeletal muscle, which interferes either directly or indirectly, with insulin signaling pathways leading to insulin resistance and cellular dysfunction $(173,179)$. Additionally, adipose tissues secrete proinflammatory cytokines and other factors such as leptin, plasminogenactivator inhibitor-1 (PAI-1), tumor necrosis factor (TNF)- $\alpha$, and interleukin-6 (IL-6) which alter the action of insulin at target cell sites via pathways that interfere with the transduction of glucose transporter 4 (GLUT-4) to plasma membranes (179). Additionally, these proinflammatory cytokines result in local or systemic inflammation which leads to disruption of beta cell function (173), thus resulting in decreased insulin production.

Diabetes is diagnosed based on fasting plasma glucose test (FPG) results of 126 $\mathrm{mg} / \mathrm{dL}$ or above, oral glucose tolerance test (OGTT) results of $200 \mathrm{mg} / \mathrm{dL}$ and above or random plasma glucose test results of $200 \mathrm{mg} / \mathrm{dL}$ or higher, in the presence of increased urination, increased thirst and unexplained weight loss (1). 


\section{Table 2.1: Disorders of Glycemic Homeostasis}

\begin{tabular}{ll}
\hline Insulin resistance & $\begin{array}{l}\text { Definition } \\
\text { A condition characterized by decreased tissue sensitivity } \\
\text { to the action of insulin }\end{array}$ \\
$\begin{array}{l}\text { Impaired glucose } \\
\text { tolerance }\end{array}$ & $\begin{array}{l}\text { Elevated blood glucose of } 140-199 \mathrm{mg} / \mathrm{dL} \text { on oral } \\
\text { glucose testing (blood glucose measured } 2 \text { hours after a } \\
75 \text {-gram oral glucose load). }\end{array}$ \\
$\begin{array}{l}\text { Impaired fasting } \\
\text { glucose }\end{array}$ & $\begin{array}{l}\text { Fasting blood glucose of } 110-125 \mathrm{mg} / \mathrm{dL} \\
\text { Diabetes Miletus }\end{array}$ \\
& $\begin{array}{l}\text { fasting plasma glucose }(\mathrm{FPG}) \text { of } \geq 126 \mathrm{mg} / \mathrm{dL} \text { or blood } \\
\text { glucose levels of } \geq 200 \mathrm{mg} / \mathrm{dL} \text { on an oral glucose } \\
\text { tolerance test }(\mathrm{OGTT})\end{array}$ \\
\hline
\end{tabular}

Diabetes accelerates atherosclerosis through a myriad of processes including endothelial dysfunction, increased vascular oxidative stress, dyslipidemia and abnormalities of platelet function, fibrinolysis, and coagulation $(5,25)$. The vascular dysfunction created by these processes is known to be intimately related to visceral adiposity, insulin resistance and hyperglycemia (25). The mechanism by which diabetes influences hemostasis, resulting in platelet and coagulation abnormalities and endothelial cell dysfunction are complex.

\section{Lipoprotein}

Diabetes, through the action of hyperglycemia and hyperinsulinemia influences changes in lipid profile through several mechanisms which predisposes to atherosclerosis. These include peripheral actions of insulin on adipose tissue and muscles, insulincontrolled apoprotein production in the liver, regulation of lipoprotein lipase (LPL), and 
the actions of cholesteryl ester transfer protein (CETP) which results in elevated production of small, dense atherogenic triglyceride-rich LDL particles (180).

Plasma Levels of non-HDL lipoprotein cholesterol and chylomicrons are increased in diabetics. Oxidation of lipoproteins is enhanced by hyperglycemia and hypertriglyceridemia (5). Oxidized lipoproteins are cytotoxic to endothelial cells and smooth muscle cells and accelerate atherogenesis (5). Additionally, hyperglycemia accelerates the conversion of LDL particles to smaller, perhaps more atherogenic, lipoproteins termed 'small-dense LDLs' (180). Furthermore, hyperglycemia facilitates the glycation of apolipoprotein B which results in impaired recognition of LDL cholesterol by its receptors on hepatocyte (5). This abnormality increases the LDL half life and thereby increases their susceptibility to oxidation and also prolongs the exposure of the endothelium to atherogenic lipoproteins $(5,180)$.

On the other hand, the glycation of HDL cholesterol during hyperglycemia reduces its half life (5). The clearance of HDL alter the lipid profile in diabetics, resulting in increased levels of VLDL, LDL and liproprotein (a)-which is a modified form of LDL(5). Lipoprotein (a) is an established risk factor for premature atherosclerotic vascular disease. Apart from it binding to endothelium and components of the extracellular matrix, it also interferes with fibrinolysis since it has a similar structure to plasminogen and competes for binding sites (5). This interference in fibrinolysis leads to the occurrence of thrombosis, which may result in vascular occlusion. furthermore, HDL cholesterol in its basal state possess enzymes that destroys oxidized lipids, but in diabetics this is mechanism is impaired by the action of acute-phase reactants (180). The 
increased glycation or inactivation of serum paraoxonase 1 (PON1) enzyme in diabetic also diminishes the ability of HDL to protect LDL from oxidation (180).

Another mechanism by which diabetes causes dyslipidemia is through the increase in production of stored fatty acids (FAs) which inhibit glucose uptake by muscle cells as well as causing increased glucose output by the liver $(180,181)$. Triglycerides levels are markedly elevated in uncontrolled diabetics or persons with hyperinsulinemia. In insulin resistance, activation of hormone-sensitive lipase (HSL) occurs, the enzyme that catalyze the conversion of stored triglycerides from adipocytes into FAs and monoglycerides which can be transferred across the plasma membrane of the cell (180, 181). The activation of HSL leads to increased production of free fatty acids (FFAs) to the liver, giving rise to hepatic overproduction of large VLDL particles and hypertriglyceridemia (180). Additionally, insulin resistance reduces the effectiveness of Lipoprotein lipase (LPL), an enzyme that plays a major role in the breakdown of triglycerides present in chylomicrons and VLDL particles, resulting in diminished triglyceride clearance from the circulation (180). The elevation of triglycerides in circulation facilitates the transport of cholesteryl esters and triglycerides between lipoproteins (180). Here, Cholesteryl ester transfer protein (CETP) modulates the collection of triglycerides from VLDL or LDL particles and exchanges them for cholesteryl esters from HDLs, and vice versa (180). However, the increased activity of CETP drives the production of small, dense triglyceride-rich LDL particles which are atherogenic (180). 


\section{Platelet and coagulation anomalies}

In diabetics, platelet adhesion and platelet aggregation are often enhanced and the balance between coagulation and fibrinolysis is greatly affected. About $80 \%$ of all premature deaths in diabetics are secondary to thrombotic events with $75 \%$ of such cases occurring in the cardiovascular system (182). Platelets in diabetics have increased expressions of activation markers, and platelet surface receptors or surface adhesion molecules including P-selectin, glycoprotein (GP) Ib and GP IIb/IIIa, which mediate binding to von Willebrand factor and platelet-fibrin interaction (183). Furthermore, in diabetics the production of nitric oxide, prostacyclin and other vasodilator prostanoids by platelets and vascular tissues, which inhibits platelet aggregation and adhesion are reduced (183).

The impaired regulations of calcium metabolism in diabetics result in increased intracellular calcium levels (183). This alteration in calcium homeostasis which leads to elevated concentration of cytosolic calcium, enhance platelet reactivity and aggregation (183). This process is suggested to provide explanation for the elevated platelet sensitivity and the increased response to variety of aggregating agents such as adenosine 5'-diphosphate (ADP), thrombin, or collagen, observed in diabetic (182).

Hyperglycemia is thought to influence platelet reactivity through several mechanisms. One of such is the non-enzymatic glycation of platelet membrane proteins that decrease membrane fluidity which may in turn also increase platelet adhesion (183). Hyperglycemia in the presence of elevated reactive oxygen species enhance the production of advanced glycation end products (AGEs) which may contribute to atherosclerosis by activating AGEs receptors (RAGE) and also by enhancing platelet 
aggregation through the serotonin receptor (183). Another possible mechanism is the activation of protein kinase $\mathrm{C}$, a mediator of platelet activation (183). Other platelet abnormalities in diabetics that increase their risk of atherosclerosis include decreased platelet survival, increased platelet generation of vasoconstrictor prostanoids, and increased glycosylation of platelet proteins (182).

Both hyperglycemia and hyperinsulinemia are known to influence coagulation. Hyperglycemia activates coagulation by raising concentrations of procoagulant factors such as tissue factor and von Willebrand factor, while inhibiting fibrinolysis by increasing concentrations of plasminogen activator inhibitor which together promotes thrombosis (183). Moreover, the increased concentration of plasminogen activator inhibitor (PAI-1) mainly due to hyperinsulinemia as well as decreased levels of antithrombin III, protein $\mathrm{C}$, and protein $\mathrm{S}$ in diabetics predisposes them to thrombosis since (PAI-1) block fibrinolysis (5).

\section{Endothelial dysfunction}

Recent advances using in vivo studies have improved our understanding of the mechanisms underlying the vascular dysfunction that leads to cardiovascular disease in persons with diabetes. The endothelium plays a vital role in vascular homeostasis by modulating vascular tone, caliber and blood flow in response to hormonal, mechanical and neural stimuli by synthesizing and secreting substances that influence vascular constriction and dilation (184). In the presence of diabetes, however, these functions are adversely impaired. Apart from maintaining vascular tone and reactivity, nitric oxide opposes the action of endothelium-derived contracting factors, inhibits leukocyte and 
platelet activation and maintains vascular smooth muscles in a non proliferative state (184). In diabetics, however, nitric oxide is either destroyed or the endothelium is insensitive to nitric oxide signaling (5). Additionally, hyperglycemia delays endothelial cell replication through both increased oxidation and glycation, causing endothelial cell. Furthermore, hyperglycemia increases the synthesis, and thereby plasma concentrations

of endothelin-1, a vasoconstrictor which causes arterial blood pressure to increase (5). All these processes lead to reduced vascular elasticity or stiffness, which in itself is an independent risk factor for cardiovascular mortality in persons with diabetes (5).

All together, these abnormalities of platelet adhesion and aggregation, coagulation and endothelial dysfunction which occur in diabetes are proposed to abolishes the cardioprotective effect of estradiol in premenopausal women (5).

\section{THE EFFECT OF SEX-SPECIFIC CHARACTERISTICS ON CVD IN WOMEN}

\section{Menopause}

Although the incidence and prevalence of CVD has been shown to increase as women enter the menopause, the mechanisms by which menopause affect cardiovascular health remains incompletely understood (40). The projected increase in the number of postmenopausal women worldwide from 467 million in 1990 to 1.2 billion by 2030 (47 million women enter the menopause each year) makes this a significant public health issue (185). Compared to men of similar age, premenopausal women have a lower risk of CVD and develop the disease 10 years later than men. Attempts to resolve this knowledge gap have resulted in several debates in the scientific community. While some 
researchers have attributed this to a chronological aging effect $(12,186,187)$, others have invoked an effect of ovarian ageing (menopause resulting in estrogen depletion) (188). This issue is further complicated by investigators suggesting that the timing of menopause- natural or surgical -influences CVD risk in women $(41,42,189)$.

Evidence for an association between the menopause and CHD originated from case-control studies in which CHD mortality was compared between age-matched groups of pre- and postmenopausal women (190). Later, prospective studies reported that the removal of the ovaries at an earlier age increased the risk of CHD, compared to women who attained natural menopause (190). Earlier, an autopsy study among 80 young oophorectomized women, compared to 80 age-matched women with intact ovaries dying from accidents or cancer reported more extensive coronary atherosclerosis and myocardial infarction in the women with oophorectomy (191). CHD risk was reduced in oophorectomized women who used estrogen therapy (191). These observations provided the basis for the widely held theory that menopause is a risk factor for heart disease, and proposal that estrogen therapy might protect women from CVD. However, a rigorous demonstration of whether menopause is a risk factor for heart disease will be to follow-up women throughout the menopausal transition and compare the incidence and mortality rates in those who become menopausal with women remaining pre-menopausal (190). Two studies which attempted this were the Framingham heart study and the Nurses' health study. The Framingham heart study which followed 2873 women for 24 years, suggested that women with menopause at a younger age had an increased risk for CHD than women whose menopause occurred later in life, although most of the women in the younger age-group had surgical menopause (192). Among women with natural 
menopause, who were aged 45 to 54 years, there was a significant increase in CHD compared to premenopausal women of similar age $(190,192)$. While the Framingham study supported menopause as a risk factor for CHD, the Nurses' Health Study, which followed 121,700 women aged 30 to 55 years for 6 years found that those women who had had a natural menopause and had never taken replacement estrogens had the same incidence of fatal and non-fatal MI as the premenopausal women $(190,193)$. In multivariable adjusted models, oophorectomy but not natural menopause increased CHD risk, and the use of estrogens appeared to eliminate this risk.

Ecological studies, which have fallen out of favor with most epidemiologists, conducted in England and Wales (12), United States (194), and Japan (186), using vital statistics data report that menopause is not a risk factor for cardiovascular disease. These cross sectional analyses of age-specific mortality do not show any proportional acceleration in heart disease at the time of menopause on a semi-logarithmic scale, and conclude that age effect rather explains the delay of CHD in premenopausal women. Cross sectional associations between age and mortality are difficult to interpret and infer association since both the exposure and outcome of interest are ascertained at the same time. Furthermore, they do not take into account the effect of changing lifestyles and treatment environments over a person's lifespan (187). Such deficiencies prompted a recent longitudinal analysis of mortality data using birth cohorts from United States, England and Wales (187). Again this birth cohort analyses did not identify any shift in CHD mortality rates at menopause but reported a linear trend, denoting an effect of chronological age (187). Such conclusions dispel the presence of an ecological fallacy playing a role in previous mortality studies. 
Other researchers have refuted such claims, and argued that the use of a semilogarithmic scale is not justifiable for the analysis of a menopausal effect on CHD mortality (188). Using mortality rates from the UK, the Framingham risk function, and estimates of relative risks at young ages from the MRFIT Study, and using a simulation model-which the authors assert makes more reasonable assumptions, Witteman et al (188), reported an increase in CHD mortality during the menopausal period, and argue that menopause indeed raises the risk of CHD in women, but did not comment whether depletion of endogenous estrogens play a role. Because all these studies analyzed only vital statistics data and therefore did not analyze individual cardiovascular risk factors or individual timing of menopause, these conclusions maybe speculative and further illustrate that vital statistics data are not a source that provide easy answers on this issue $(41,187)$.

Whether the increased risk of CVD in women is a function of chronological age, ovarian aging associated with depletion in endogenous estrogens or both is methodologically challenging since these two concurrent processes are intimately linked, and will continue to be a debated issue (195). This significant gap in knowledge has grave consequences in the prevention and care of women with heart disease.

During the menopausal transition, several changes in the lipid profile occur which tend to favor the atherosclerotic process (figure 2.1). Results from the Study of Women's Health across the Nation (SWAN) show that total cholesterol, low-density lipoprotein (LDL) cholesterol and apolipoprotein-B levels rise slowly through the premenopausal years, and increase rapidly during perimenopause until plateauing after menopause, a pattern consistent with menopause-induced changes but not chronological age (40). This 
observation is similar across ethnic groups. Additionally, after menopause, LDL particle distribution shifts to smaller particles which are denser and more atherogenic (66). At the same time, changes in HDL subclass are observed, with a decrease in HDL2 levels concurrent with an increase in HDL3 levels (66). These HDL particles, even in healthy postmenopausal women, have been observed to have redacted ability to inhibit LDL oxidation (196).

Besides these modifications in lipid profile of women transitioning to menopause, current evidence confirms that changes in body fat distribution occur during this time period (197). This is characterized by a shift of fat mass from the legs towards the upper body even in the absence of significant changes in total body fat (197). In healthy premenopausal women, fat is deposited mainly in the gluteofemoral region-termed gynoid adiposity $(198,199)$. However, during menopause, fat is accumulated in the abdominal region-termed android adiposity $(198,199)$. Data from 1988-1994 show that the national prevalence of abdominal obesity (as defined by waist circumference $>88$ $\mathrm{cm}$ ) was reported to be $22.8 \%$ among women aged 20-29 compared to $67.3 \%$ among those aged 60-69 (200). These changes in lipid profile and body composition are thought to be initiated and moderated by sex hormones and insulin resistance hyperinsulinemia. The influence of sex hormones on these processes will be described in detail.

\section{Surgical Menopause}

While the gradual loss of ovulation, estrogen and progesterone production, known as the climacteric occur over about 10 years and culminate in the cessation of ovulation known as menopause at a median age of 51 years, induced/surgical menopause 
occurs when the ovaries are surgically removed by bilateral oophorectomy or are damaged by radiation- or chemo-therapy. This more abrupt loss of ovarian function and decline in plasma estrogen levels is proposed to influence long-term health outcomes. Bilateral salpingo-oophorectomy (BSO) is commonly offered concomitant with hysterectomy as a prophylactic procedure to prevent ovarian cancer, or as a treatment for premenstrual syndrome and symptomatic endometriosis or other conditions (201). When normal ovaries are removed at the time of another procedure, it is termed prophylactic or elective oophorectomy (202). Hysterectomy, the removal of the uterus is the most common major surgery performed among non pregnant women in the United States (49, 203). Of the more than 600,000 hysterectomies performed annually, approximately $54 \%$ include BSO $(49,203)$. The proportion of women with BSO increased from 25\% in 1979 to $45 \%$ in 2004 (202). However at the turn of the century, an overall decrease in the rate of BSO has been observed nationwide. Since, hormone therapy was commonly prescribed for all women undergoing oophorectomy, this declining trend in BSO rate may reflect the impact of recent publications about the adverse effects of hormone therapy on patients' and physicians' decision-making (202).

During the period of 2001 to 2006 , an $8 \%$ overall reduction in the incidence of oophorectomy was reported, with a relative reduction of 35\% among women younger than 55 years (204). However, $63 \%$ of BSO were performed in women aged 45 to 49 years compared to $30 \%$ in women aged 35 to 39 years (205) Women who live in the South are more likely to undergo oophorectomy (42\%), an observation that reflects geographic variation in practice patterns and/or patient preferences (202). 


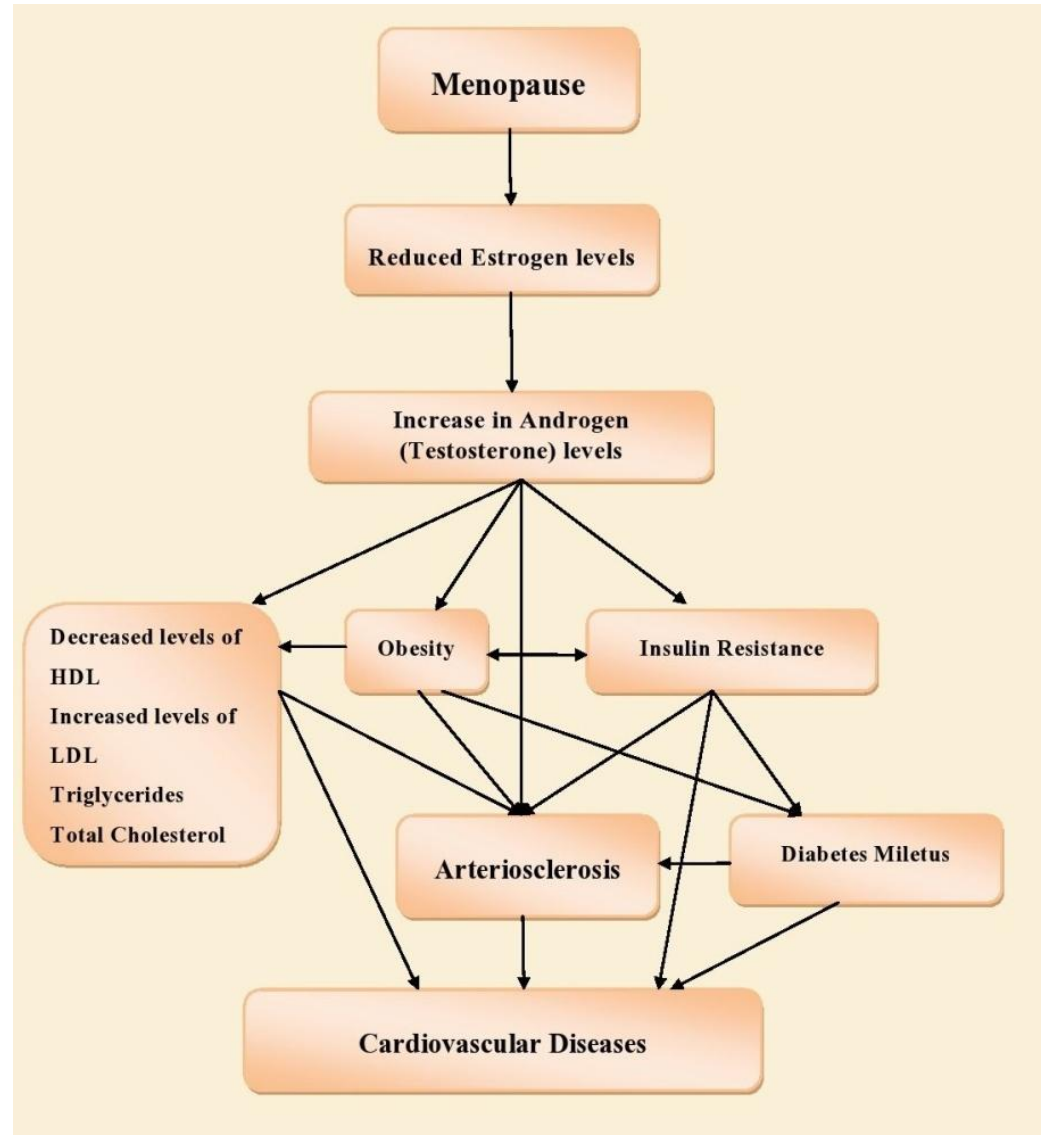

Fig. 2.1 The mechanism of menopause and cardiovascular disease

Although race is not a significant factor for $\mathrm{BSO}$, a greater proportion of women with BSO are non-Hispanic Whites, while black/African American women are more likely to undergo hysterectomy (202). Regardless, socioeconomic status plays a role in the choice and age of BSO (204). In younger women, those who are uninsured or are enrolled in Medicaid programs are more likely to undergo BSO. On the contrary older women with private or self-paid for insurance are more likely have an oophorectomy. Younger women who choose to have an oophorectomy before the average age at natural menopause may not all be truly undergoing prophylactic surgery, but instead may partly represent a select group of women with high risk for worse health outcomes, since they more likely to have 
a family history of breast or ovarian cancer, as well as a personal history of breast cancer, ovarian cysts, or endometriosis at the time of surgery (202).

Results from prospective studies suggest that there is equipoise about the risks and benefits of bilateral oophorectomy especially in women with normal ovaries. Despite its benefits in decreasing the risk of epithelial ovarian cancer, breast cancer, and a decrease in pelvic pain and future pelvic surgery risk, oophorectomy is asserted to have deleterious effects on cognitive function, cardiovascular and bone health $(202,204)$. The debate about risks and benefits of prophylactic oophorectomy has led to several revisions in recommendations by the American College of Obstetricians and Gynecologists (204). Furthermore, the effect of elective oophorectomy on cardiovascular outcomes remains controversial and existing evidence is inconclusive to determine risk or benefit (58). In our view, several methodological limitations exist among studies conducted to demonstrate whether there is an association between BSO and CVD (58). Notably, women who undergo hysterectomy (regardless of ovarian status) have adverse CVD risk profile compared to those without hysterectomy (57), and a number of studies have failed to control for these factors. Additionally, others did not control for the use of HT, which has been suggested to have a short term beneficial effect when initiated immediately after the surgical procedure in premenopausal women (63). Other methodological factors that have resulted in conflicting conclusions concerning the effect of BSO and CVD include inappropriate comparison group, small sample size and inadequate methods to address confounding (63). These methodological differences among studies limit their synthesis for meta-analysis (63). Nevertheless, in a meta-analysis by Atsma et al (41), the risk of CVD was reported to be 4-fold among women with oophorectomy. In view of the small 
sample size (3 studies) and substantial heterogeneity among studies in their stratified analyses of bilateral oophorectomy, care should be taken in interpreting these results.

Two of the largest cohorts to address the effect of BSO on incident cardiovascular disease and mortality were the Nurses' Health Study and the Women's Health Initiate. Parker et al (53) examined the records of 29,380 women followed for 24 years in the Nurses' Health Study, and reported that compared with ovarian conservation, BSO at the time of hysterectomy increased the risk for all cause mortality (HR: 1.12, 95\% CI: 1.031.21), as well as fatal and nonfatal CHD (HR: $1.17,95 \%$ CI: 1.02-1.35) but this increased risk was limited to women with their ovaries removed before age 45 years. The strength of that study does not only lie in the appropriate comparison group for BSO, but also the extensive adjustment for confounders (age, age at hysterectomy, diabetes, high blood pressure, smoking status, hypercholesterolemia, family history of myocardial infarction before age 60 years, body mass index, use of estrogen therapy, duration of oral contraceptive use, parity, average daily alcohol consumption, total hours of weekly physical activity, and acetylsalicylic acid use)

Contrastingly, Howard et al (57), using data from 89,914 participants of the WHI Observational Study reported no significant association between BSO and fatal and nonfatal CVD after adjusting for several confounding variables (HR: 1.11, 95\% CI: 0.99 1.24) when compared to women with no hysterectomy or oophorectomy. These results were later confirmed by Jacoby et al (59) using a sample of 25,448 women aged 50 to 79 years with a history of hysterectomy from the same cohort. Comparing participants with both hysterectomy and BSO to women with ovarian conservation, BSO was not associated with an increased risk of fatal and nonfatal coronary heart disease (HR:1.00, 
CI: $0.85-1.18$ ), stroke (HR:1.04, CI: $0.87-1.24)$ or total cardiovascular disease (HR:0.99, CI: 0.91-1.09) after adjusting extensively for confounders such as age, race/ethnicity, educational level, medical insurance, parity, body mass index, HT status, smoking, alcohol use, physical activity, hypertension, diabetes mellitus, hypercholesterolemia and personal or family history of angina, myocardial infarction, stroke or any percutaneous coronary intervention procedure. The same outcome was also seen among women with BSO and no history of HT use, suggesting that BSO of itself may not incur additional risk for CVD, especially among the WHI cohort.

Several differences among these two cohorts are worth mentioning which might influence the divergent results reported. While both enrolled a greater proportion of NonHispanic whites, the Nurses' Health Study had 94\% compared to $85 \%$ in the WHI study $(53,57)$. Furthermore the Nurses' Health Study cohort were homogeneous with regard to education, socioeconomic status and access to care, while the WHI cohort was heterogeneous for these above mentioned factors, older (mean age of 63 vs. 51 years) and had less follow-up time ( 8 years vs. 24 years) $(53,57)$.

One hypothesis is that the abrupt reduction in endogenous estrogen levels without HT therapy among women with oophorectomy raises their risk for CVD. This idea is supported by results from a sub-study of the WHI estrogen alone trial in which no significant associations between BSO and subclinical coronary artery disease was reported (50). In women with BSO without hormone therapy, however, a 2 fold increased risk (OR: 2.0, 95\%CI: 1.2-3.4) for atherosclerosis, as assessed by coronary artery calcium (CAC) scores was identified independent of traditional CVD risk factors. This 
underscores the complex biologic effects and multiple pathways by which estrogen influences the atherosclerotic process.

\section{Polycystic ovary syndrome}

Polycystic ovary syndrome (PCOS) is an incompletely understood hormonal disorder which is characterized by enlarged cystic ovaries, chronic hyperandrogenemia, irregular or absent menstrual cycles, hirsutism, insulin resistance and obesity (206). It is

the most common endocrinopathy in women of childbearing age (207). The prevalence of PCOS varies depending on the diagnostic criteria used (207). PCOS prevalence of 6-10\% is reported when applying the National Institute of Health criteria, while this estimate increases to $20 \%$ when using the Rotterdam criteria; however, this number may significantly under represent the prevalence since many cases are not recognized (207, 208). Apart from hyperandrogenemia, several other CVD risk factors are common in women with PCOS including hypertension, low HDL cholesterol, as well as elevated levels of triglycerides, LDL cholesterol, and homocysteine $(207,208)$, and as many as $70 \%$ of women with PCOS are obese (209). Numerous studies have reported that PCOS is associated with diabetes, atherosclerosis and CVD.

Using electron beam computed tomography measure coronary artery calcium noninvasively, Christian et al (210), reported that women with PCOS have a greater prevalence of subclinical coronary artery disease than obese or non-obese women of similar age. Compared to age-and BMI-matched healthy, ovulatory controls, the risk of atherosclerosis in women with PCOS aged 30 to 45 years was five-fold (OR: 5.89, 95\% CI: 2.46-13.97) (210), even after excluding participants with diabetes and prevalent 
CHD. Additionally, other studies reported that body weight and obesity do not fully explain the increased atherosclerosis risk in women with PCOS as assessed by carotid intima-media wall thickness (211). Furthermore, reports from the Coronary Artery Risk Development in Young Adults (CARDIA) study demonstrates that PCOS women have higher left ventricular mass index and left atrial diameter (212) compared to healthy controls. Likewise the Women's Ischemia Syndrome Evaluation (WISE) Study reported an increased risk of angiographic CHD (OR 1.88, 95\%CI: 1.06-3.40) in women with PCOS compared to controls (213).

On the other hand, observational studies to assess the incidence of cardiovascular morbidity and mortality in PCOS patients have produced conflicting results. Iftikhar et al (207), found no statistically significant risk in the incidence of MI, CVD or stroke related mortality, among 309 women with PCOS followed for 24 years in Olmsted County, Minnesota. On the contrary, a 20 year follow-up of 2,301 multiethnic British women including 29\% Asians with PCOS identified a higher incidence of diabetes, MI and angina in women with PCOS than in the national female population (214). Additionally, the increased risk was limited to women aged 45 years and older with over a quarter having MI or angina after age 65 years (214). To try to resolve the ambiguity in the association of PCOS with CVD hard endpoints and to assess if obesity mediated this relationship, a meta-analysis of controlled observational studies was conducted by de Groot et al (209). Using a random effects model, the relative risk for CHD or stroke was 2.02 comparing women with PCOS to women without PCOS (95\% CI: 1.47-2.76). Although the risk was attenuated by controlling for the effect of BMI, it still attained statistical significance (RR: 1.55, 95\%CI: 1.27-1.89). 
Statistical adjustment for traditional risk factors of CVD does not fully explain the excess risk of CVD among women with PCOS. However, women with PCOS are more likely to have central adiposity, insulin resistance and hyperandrogenemia as do women with diabetes (215). Since both groups of women share these risk factors and have more CVD, these factors may explain the excess CVD risk in these two specialized populations.

\section{SEX HORMONES AND CARDIOVASCULAR DISEASE}

The role of sex hormones in women's cardiovascular health is an emerging field of interest (96). Failure to demonstrate that exogenous estrogens are effective in the primary or secondary prevention of CVD in both the HERS and WHI trials, has brought increasing attention to the role of other hormones in cardiovascular health outcomes. One idea is that testosterone predisposes older women and those with PCOS or diabetes to increased CVD risk. Accordingly, among younger women, estrogen opposes the detrimental effects of testosterone on chronic cardiac remodeling and function (216) by direct effects and by increasing the production of SHBG which reduces the portion of the circulating testosterone that affects target tissues (bioavailable testosterone). After menopause estradiol levels are dramatically reduced whereas testosterone levels fall only modestly allowing testosterones and other androgens to adversely impact CVD risk. Furthermore, estrogen deficiency in women predisposes to visceral fat accumulation which is strongly associated with insulin resistance. Following the menopause, women show a steeper increase in visceral fat than men, perhaps because of estrogen deficiency, 
and the increase in androgen production that occurs with increased visceral fat may increase CVD risk especially in women with PCOS and diabetes.

\section{ESTROGEN}

\section{Estrogen production and metabolism}

The ovaries are the main source of estrogen production in premenopausal women, and estradiol is the major and most important circulating estrogen which acts on target tissues throughout the body (217). Estrogens are produced from cholesterol by a series of biotransformations which culminate in bioconversion of testosterone to estradiol by the enzyme aromatase (217). This enzyme is expressed in other tissues, however, allowing for estrogen production in the liver, adipose cells in the breast and other parts of the body, osteoblasts , chondocytes, muscle cells and skin cells and the placenta during pregnancy (217). In postmenopausal women, these sites of peripheral production of estrogen become the major source of estrogen since the ovaries cease producing estrogens (217). At this time, estrone is the primary estrogen in the circulation and is produced by the bioconversion of androstenedione from the adrenal cortex (218). A very small amount of estradiol is formed through the extragonadal conversion of testosterone (218). Adipose tissue is a significant site for the conversion of androstenedione to estrone in postmenopausal women, and the level of estrone in plasma is directly proportional to BMI (218).

After release from the ovaries and peripheral tissues, estradiol circulates bound to albumen (60\%), sex hormone binding globulin (SHBG) (38\%), or unbound (2\%) 
(219). Estrogens are metabolized primarily in the liver in two phases $(220,221)$. Phase I is hydroxylation of molecule at the 2nd, 4th and 16th alpha carbon position by the addition of a hydroxyl ion yielding 2-hydroxyestrone, 4-hydroxyestrone and 16-alphahydroxyestrone respectively $(220,221)$. It's now known that 2-hydroxyestrone has beneficial effect while 4-hydroxyestrone and 16-alpha-hydroxyestrone lead to tissue proliferation which promotes carcinogenesis $(220,221)$. These hydroxyestrones (catechol estrogens) are then oxidized to quinones (phase II). Quinones from 2-hydroxyestrone and 4-hydroxyestrone are also known to have a deleterious effect on DNA either directly or indirectly through the generation of reactive oxygen species $(220,221)$. They are methlylated by catechol-O-methyltransferase (COMT) to detoxify them for excretion $(220,221)$. Methylated catechol estrogens are conjugated with glucuronic acid to facilitate their excretion in the feces and urine $(220,221)$. In the intestines, certain bacteria produce the glucuronidase enzymes which break the bond between the estrogen and glucuronic acid, allowing the estrogens to re-enter the blood stream $(220,221)$.

\section{Protective Effects of Estrogen on the Cardiovascular System}

Endogenous estrogens have a cardio-protective effect via several mechanisms. Estrogens increase HDL cholesterol levels and reduce LDL levels (96). However, estrogen- induced changes in the lipid profile account for only about one third of its cardio-protective effects (222). Additionally, estrogen activates the release of the vasodilators nitric oxide and prostacyclin from vascular endothelial cells to inhibit vascular constriction and thereby reduce arterial blood pressure (96). The increased production of nitric oxide also reduces platelet aggregation (96). Furthermore, estrogen 
inhibits endothelial cell apoptosis, promotes increased endothelial cell proliferation and angiogenesis. The route of administration of exogenous estrogens has differential effect on lipid levels, with estrogens administered transdermally having less effect on plasma lipids compared to orally administered estrogen (96). Estrogens administered transdermally produce higher concentrations in plasma with a more rapid onset of action than estrogens administered orally as a result of the first pass effect, however estrogens administered orally increased the expression of many genes in liver, clotting factors, and sex hormone binding globulin (223)..

\section{Hormone Therapy and Cardiovascular disease}

Earlier autopsy studies in oophorectomized women receiving hormone treatment or none reported that estrogen has a positive effect on the coronary vasculature (191). Supported by experiments in vitro and in experimental animal, it was asserted that endogenous estrogens might have a protective effect on the primary prevention of CVD in women. This idea seems plausible since premenopausal women are less likely to have a CVD event compared to men of similar age, and CHD risk is increased in postmenopausal women (224). A meta-analysis of observational studies published up until 1996 reported a protective effect of HRT among ever users from CHD and CHDrelated mortality, with Barrett-Connor et al (225), reporting a statistically significant reduced risk of $30 \%$ and $34 \%$ for unopposed and opposed therapy respectively. However two large randomized clinical trials, the WHI Study and the HERS study, reported results that were contrary, and refuted the theory that estrogens are protective in the primary prevention of CVD in postmenopausal women (60-62). 
The publications of the results of those trials lead to extensive debate with many scientists arguing that women enrolled in the primary prevention trial (WHI) were older and initiated hormone therapy years after the onset on menopause, unlike earlier observational studies which enrolled women closer to the time of menopause. This notion was supported by experimental studies in monkeys in which hormone therapy was effective in slowing atherosclerosis when administered soon after surgical menopause whereas the benefit was lost if therapy was delayed until plaques had developed (226) This prompted further analyses of the combined data from both the observational and clinical trial arms of WHI according to age and years since menopause. While women who initiated therapy closer to menopause were observed to have reduced CHD risk compared to increase CHD risk among women more distant from age at menopause, these results did not meet statistical significance (227).

The National Heart, Lung, and Blood Institute-sponsored Women's Ischemia Syndrome Evaluation (WISE) study which followed 654 postmenopausal for a median of 6 years reported lower prevalence of obstructive CAD (OR: 0.44, 95\%CI: 0.30-0.73) and CVD (HR: 0.60, 95\% CI: 0.41-0.88) among hormone therapy users who had natural menopause and were less than 55 years (228). Similarly, three meta-analyses of randomized controlled trials seem to affirm the timing hypothesis by concluding that a significant reduction in CHD and mortality was observed in women who were randomized to HT before age 60 or within 10 years of menopause (229-231). One of such was a Bayesian meta-analysis that included women at a mean age of 55 years and reported a $27 \%$ reduced risk of mortality by HT (RR:0.73, 95\%CI: $0.52-0.96)$ in younger postmenopausal women (231). Recently, a clinical trial which enrolled 1006 healthy 
newly postmenopausal women aged $45-58$ who were randomized to either triphasic estradiol and norethisterone acetate (if they had intact uterus) or $2 \mathrm{mg}$ estradiol a day if they had undergone hysterectomy, reported a 52\% reduced risk of CVD events (a composite endpoint of mortality, MI, or heart failure) by hormone therapy (HR: 0.48, 95\%CI: 0.26- 0.87) (232). Although this is the first randomized trial to study healthy women treated early in menopause with $17-\beta$-estradiol and norethisterone acetate, as well as the longest HT intervention with 10 years of follow-up, it was however an open label trial with no placebo or blinding (232).

The timing hypothesis remains controversial; however the protective effect of estrogens in perimenopausal or early postmenopausal women for the treatment of menopausal symptoms which may in turn slow down the progression of atherosclerosis seems convincing (226). Taken together, these observations seem plausible since newly menopausal women have fewer thrombotic modifications to their vasculature due to healthier vascular endothelium which is also more resistant to thrombosis (96), and thereby might benefit from the protective effect of estrogen.

\section{ANDROGENS}

\section{Androgens production}

The postmenopausal ovary is an ongoing site of androgen production. While both circulating estrogens (mainly estradiol) and androgens levels fall with menopause due to ovarian follicular depletion and age-related atrophy of the adrenal cortex, there is a drastic reduction in estradiol compared to testosterone, resulting in a more androgenic 
milieu in older women (35). A reduction in SHBG levels during menopause also fosters this excess androgenic state (233). Although postmenopausal ovaries cease producing estradiol, the ovarian stroma, however, continues to produce testosterone (234). Thus women with bilateral oophorectomy have lower levels of androgens (50\% less) compared to women with natural menopause $(36,37)$, which persist even after age 65 years $(234)$. Meanwhile, postmenopausal women with unilateral oophorectomy have significantly higher levels of androstenedione and testosterone than menopausal women with bilateral oophorectomy (202). Certain factors promote reduced circulating levels of testosterone. Estrogen therapy administered orally increases SHBG production (approximately 75\%) which results in reduced levels of free and total testosterone (233). Evidently, transdermal hormone therapy has to be administered at very high dose to achieve this same effect (233). Other conditions and therapies including Addison's disease, corticosteroid therapy, chronic illness, and premenopausal ovarian failure are all known to decrease circulatory levels of androgens resulting in androgen deficiency (233).

There are five major androgens produced in women. Listed in descending order of serum concentration, they are dehydroepiandrosterone sulphate (DHEAS), dehydroepiandrosterone (DHEA), androstenedione, testosterone, and dihydrotestosterone (DHT) (233). DHT and Testosterone are the most potent androgen (233), while DHEAS, DHEA, and androstenedione can be converted to testosterone (233). The biosynthesis of androgens is modulated by two cytochrome P450 enzymes. P450 SCC modulates cholesterol side chain cleavage, while P450 c17 catalyzes 17-hydroxylation and 17-20 bond cleavage to form DHEA and androstenedione (233). The conversion of DHEA to androstenedione is catalyzed by the enzyme $3 \beta$-hydroxy steroid dehydrogenase ( $3 \beta$ - 
HSD), and 17ß-hydroxy steroid dehydrogenase (17 $\beta$-HSD) catalyzes the conversion of androstenedione to testosterone (233). About $65 \%$ to $75 \%$ of testosterone in the plasma is bound to SHBG, $1-2 \%$ is unbound, and the remaining $25 \%$ to $35 \%$ is bound loosely to albumin (235).

In women, androgen production takes place in three compartments: the ovary, adrenal cortex and in peripheral tissue (236). In premenopausal women, 25\% of testosterone is derived from the ovaries (35). Another 25\% is produced by the adrenal cortex through the stimulation of adrenocorticotropic hormone (ACTH), and 50\% is from peripheral conversion of androstenedione, DHEA and DHEA-S (35). In postmenopausal women, however, the sources of testosterone changes with 50\% from the ovaries, $10 \%$ from the adrenal cortex and $40 \%$ from peripheral conversion (35). (Figure 2.2)

Follicular depletions results in reduced estradiol secretion which leads to loss of negative feedback to the hypothalamus and pituitary gland, leading to increased production of luteinizing hormones (LH) which stimulates ovaries to produce testosterone (235).

There is a positive association between the levels of insulin and testosterone in plasma, which is exaggerated in women with PCOS or diabetes. Insulin stimulates ovarian production of testosterone, and insulin resistance is associated with low levels of SHBG such that diabetics having higher circulating levels of testosterone compared to normal women of the same age (36). Furthermore, in the ovaries of premenopausal diabetic women, conversion of androgens to estrogens less effectively due to decreased ovarian aromatase activity (94), perhaps because of increased mullerian inhbitory hormone.. 


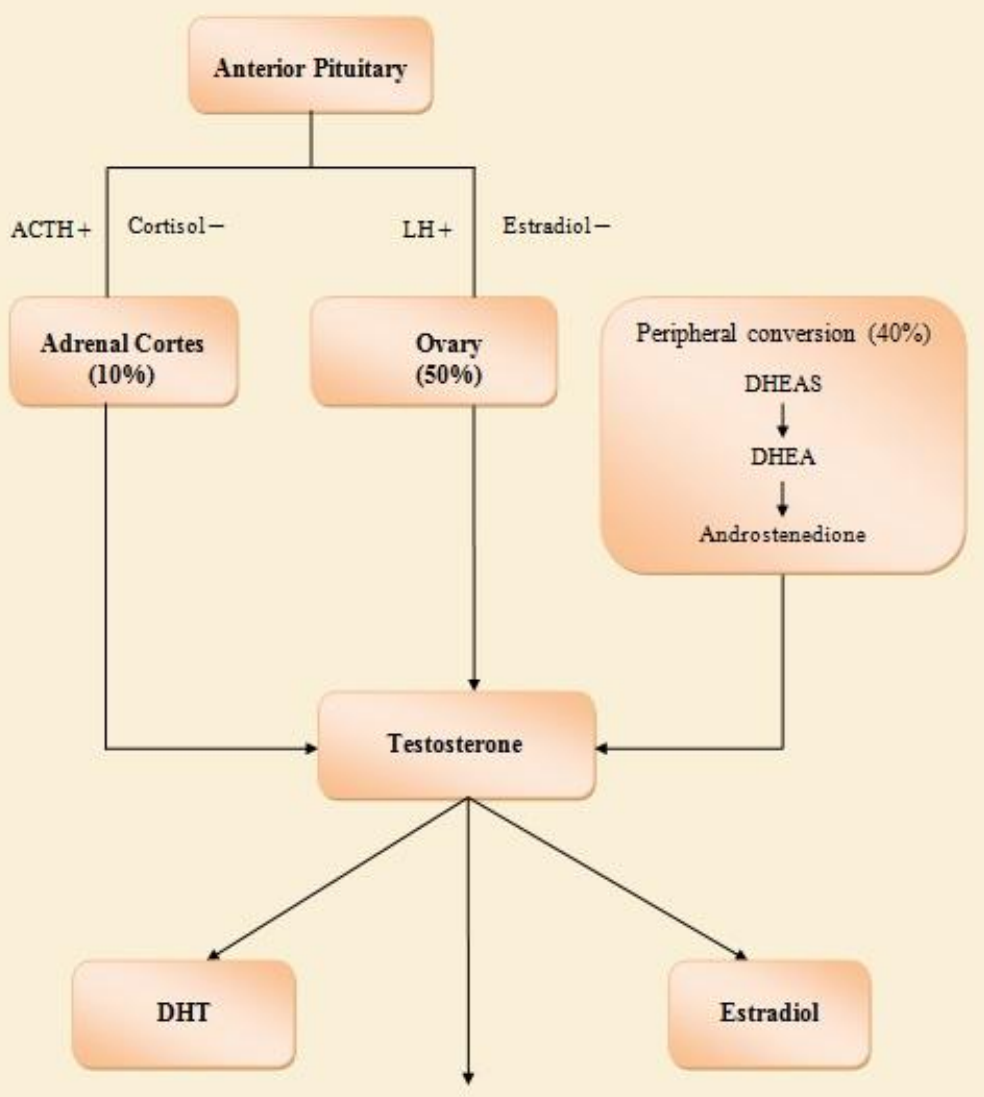

Fig 2.2 Sources of testosterone production in postmenopausal women

This imbalance between estradiol and testosterone may be one mechanism for the increase in CHD risk in diabetic women (94). It is insulin that stimulates elevated ovarian androgen production and not androgens causing insulin resistance and hyperinsulinemia (94), since in hyperinsuulinemic hyperandrogenic women with PCOS, suppression of luteinizing hormones with a gonadotropin-releasing hormone $(\mathrm{GnRH})$ analogues reduced androgen levels without altering insulin sensitivity or circulating insulin levels (94). 
However, among PCOS women whose insulin levels-declined through diet or drugs, a fall in serum androgens was observed $(94,237)$.

\section{Androgens as a Cardiometabolic risk factor}

\section{Adiposity and fat distribution}

There is a large body of scientific literature on the influence of androgens on the connection between androgens and the accumulation and distribution of fat in postmenopausal women independent of age, total fat mass and other CVD risk factors $(67,198,199,238-240)$. In the Study of Women's Health Across the Nation (SWAN) Fat Patterning Study, the level of bioavailable testosterone was associated with the amount of visceral fat even after adjusting for insulin resistance, suggesting that testosterone is linked to regional fat distribution (67). This is an important relationship since both factors are associated with higher cardiovascular risk in women (figure 2.3). Obese women have elevated circulating androgens levels, especially bioavailable testosterone, and DHEAS, as well as an increased androgen production rate $(94,241)$. Furthermore, testosterone clearance is accelerated in obese individuals due to lower SHBG (239), while the causeand- effect relationship between androgens and visceral adiposity is complex. Female-tomale transsexuals, testosterones treatment increased BMI and visceral adiposity (35).

Android obesity is associated with a greater risk for metabolic syndrome, diabetes and CVD in women than is gynoid obesity (242). The action of visceral fat and its deleterious effect on CVD is poorly understood. However, the amount of abdominal obesity correlates positively with small dense LDL particles, reduced HDL, insulin resistance, hyperinsulinemia, growth hormone deficiency and hypertension, independent 
of age and total body fat or body mass index (BMI) (74, 243-246). Visceral adipose tissue (VAT), is known to be more metabolically active than subcutaneous adipose tissue (SAT) and is linked to increased insulin resistance.(16, 242, 247, 248). Additionally, VAT secretes larger quantities of pro-inflammatory cytokines such as TNF - $\alpha$ and interleukin-6 which increase the level of the acute phase reactant C-reactive protein (242, 247), and contributes to atherothrombosis through the activation of intravascular coagulation and fibrinolysis (249). Finally VAT may directly influence atherosclerosis by accelerating the progression of coronary calcification (71).

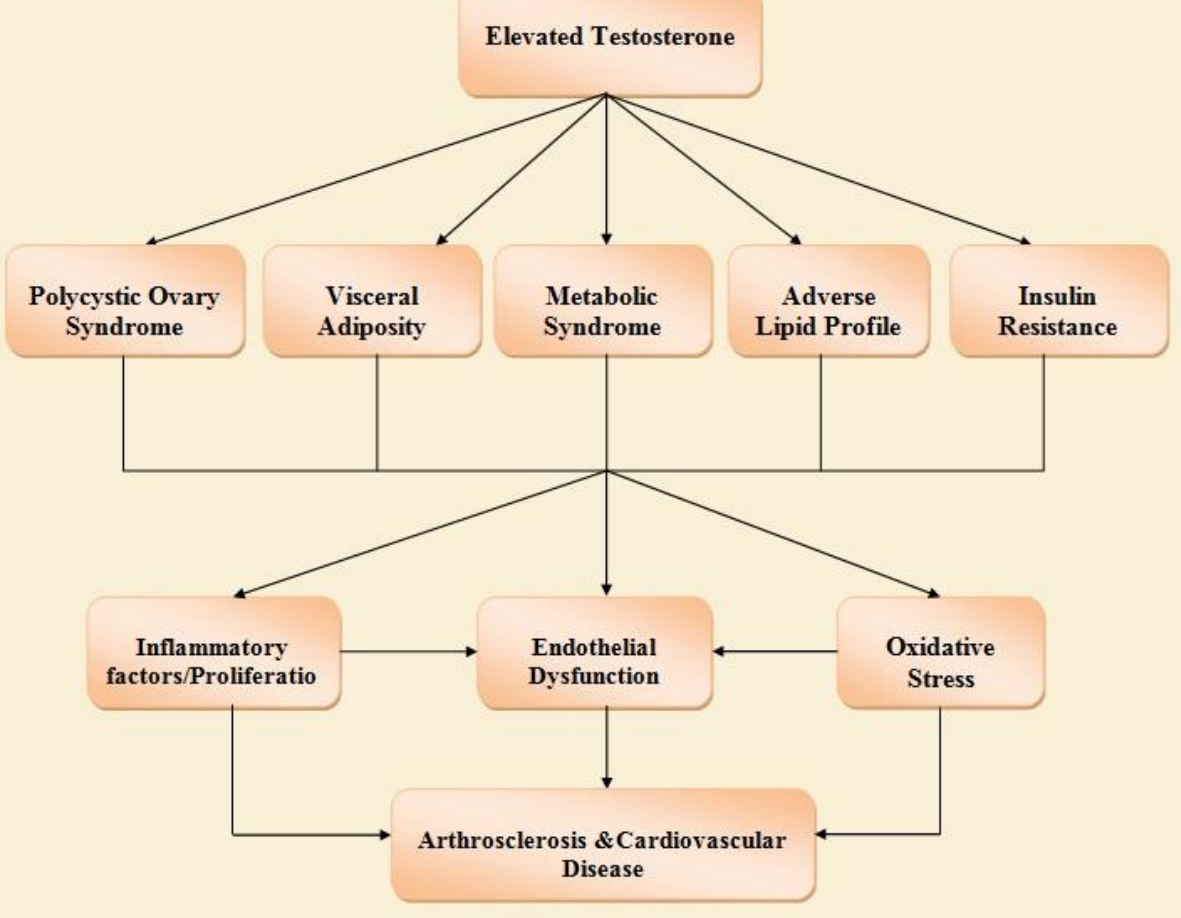

Fig. 2.3 Actions of elevated testosterone on cardiovascular risk and disease 
Several studies have found that bilateral oophorectomy, when performed in premenopausal women, increases their risk for CVD, but the explanation for this important association is uncertain. While no statistically significant relationship between BSO and all-cause and cause-specific mortality was reported among participants in the third National Health and Nutritional Examination Survey obese women who underwent BSO before 40 years of age had an increased risk of mortality, with the lack of HT use compounding this effect. Studies in rodents reveal that BSO increases visceral fat content (250-252), LDL cholesterol, and produced a hyperinsulemic response to glucose tolerance implying insulin resistance which is partially reversed with estrogen supplementation $(251,252)$. While no comparable data are available in humans, if estrogens decrease visceral fat accumulation in humans, then removal of the ovaries at an early age may have a more adverse effect on CVD risk than if this procedure is performed after the time of natural menopause when estrogen production is low (234, 253). Moreover, the increase in visceral fat may stimulate androgen production to further increase CVD risk.

\section{Lipid Profile}

Clinical observations suggest that testosterone plays an important role in lipid metabolism. For instance among female-to-male transgender patients who were receiving testosterone therapy, a reduction in HDL cholesterol was identified, coupled with elevated triglycerides. When obese postmenopausal women were administered nandolone deconoate (an anabolic steroid) in a 9-month randomized placebo-controlled trial (254), 
HDL cholesterol levels were reduced but no significant change in fasting glucose or insulin sensitivity was observed (254).

Androgens may influence lipid metabolism through several mechanisms. These include increasing the activity of lipoprotein lipase and hepatic triglyceride lipase which results in elevated levels of triglyceride in adipose tissue, as well as a decrease in total circulating high-density lipoprotein levels (255). Furthermore, androgens may decrease catabolic removal of low-density lipoproteins from circulating plasma (255).

A study to characterize the cross-sectional relationship between endogenous hormones and lipid parameters in 328 postmenopausal women with prevalent carotid atherosclerosis in the Atherosclerosis Risk in Communities Study reported a positive correlation between free androgen index and increased levels of triglyceride, LDL and total cholesterol (69). Although free androgen index was associated with a more atherogenic lipid profile independent of demographic, metabolic factors and health behaviors, SHBG on the other hand was statistically significantly associated with a more favorable lipid profile (69). Another study of 598 healthy Greek postmenopausal women not on hormone therapy demonstrated that compared to women in the lowest testosterone quartile, women in the highest testosterone quartile had higher levels of total cholesterol, LDL cholesterol, apolipoprotein B and decreased HDL cholesterol, independent of the effect of age, BMI, insulin resistance and social habits (70). Interestingly, the free estrogen index exhibited similar associations with lipid profiles as the free androgen index (70).

Although, endogenous androgens, namely testosterone has been shown to also increase levels of total and LDL cholesterol, reduce HDL cholesterol in most 
observational studies (68-70), results from other studies do not support a major role of testosterone in lipid metabolism. Stork et al (256), studying 889 healthy postmenopausal participating in the PROSPECT study did not identify any association between total testosterone and lipid metabolism, however free androgen index was weakly inversely correlated with HDL cholesterol levels. Furthermore, a more androgenic profile was associated with high levels of C-reactive protein (CRP), after adjustment for age, traditional cardiovascular risk factors and markers of body composition. Adipose tissue is known to produce inflammatory mediators that increase CRP-production in the liver (1). However, a positive association between androgens with a nonspecific inflammation marker like CRP independent of adiposity was still reported (1). This suggests that androgens may independently influence the inflammation process during atherosclerosis.

\section{Subclinical coronary disease}

The impact of androgens on subclinical coronary artery disease is largely unexplained; however, experimental studies suggest that androgens may promote several mechanisms of the atherosclerotic process. Recent evidence acknowledges that testosterone influence the process of inflammation by increasing the level of endothelin 1 (ET-1) which is a vasoconstrictor and is usually present at early stages of endothelial dysfunction, while 17ß-estradiol reduces ET-1 levels (257). Testosterone leads to apoptotic damage of the endothelial cells under serum deprivation, leading to endothelial leakage, increased platelet adhesiveness and thrombus formation (258). This results in clot formation that block narrow arteries, cutting off oxygen supply to the heart and myocardial infarction when the coronary arteries are occluded (258). In vitro studies also 
demonstrate that testosterone increases monocyte adhesion to the vascular endothelium (259). Similar to aging, hyperandrogenicity results in increased oxidative stress and advance glycation end-products (AGE) which are well-documented atherogenic molecules that interact with the transmembrane receptor RAGE, leading to the activation of inflammation, oxidative stress and proatherogeneic cascades (260).

\section{The Effect of Androgens on Cardiovascular disease outcomes}

Little is known about the association of androgen levels with cardiovascular disease outcomes in postmenopausal women, and the few studies conducted reveal conflicting results. In some instances, both $\mathrm{J}$ and $\mathrm{U}$-shaped relationships have been proposed, while in others, either a reverse association of testosterone with CVD outcomes or null associations have been reported. Table 2.2 summarizes the reported association of androgens with cardiovascular events.

The Rancho Bernado study (261), reported no association between bioavailable testosterone levels and CVD mortality in 651 post-menopausal women while another cohort study of 2914 German women in primary care reported that women with low total testosterone levels had increased CVD mortality (262). The risk of CVD-related mortality was elevated for participants with total testosterone levels in the lowest quintile (Q1), with patients in the collapsed quintiles (Q2-Q5) having a 32\% reduced risk (HR 0.68, 95\%CI: 0.48-0.97) (262). Three were no results for SHBG or bioavailable testosterone.

Conversely, Shaw et al (213) reported a 59\% increased risk of CVD mortality among postmenopausal women with a history of irregular menses as well as clinical features of PCOS. These women were both hyperandrogenic and insulin resistant. 
A large nested case control study, the Women's Health Study, found that among women not using hormone therapy, there was a higher calculated free androgen index (FAI) among postmenopausal women who developed CVD events. This did not attain statistical significance after controlling for the independent effect of body mass index and other cardiovascular risk factors, however (263). The study by Juutilainen et al (9) which examined free testosterone levels- the biologically active form- found no association with either CVD or all cause mortality, except among diabetic women. However the external validity of that study is limited since it was not population based but was instead conducted among women referred for coronary angiography, and results may not be generalisable to women with low cardiovascular risk.

It is necessary to highlight some of the limitations in studies undertaken to demonstrate association between androgens namely testosterone and CVD. In non prospective studies (i.e. cross-sectional studies), reverse causality cannot also be ruled out. Additionally study populations were heterogeneous and selection criteria were diverse (35). Adjustments for confounders were not always adequate in some studies to control for spurious associations. Furthermore in case control studies, bias may be introduced since atherogenic changes may cause a decline in testosterone levels in participants diagnosed with CVD (35).

\section{THE EFFECT OF ANDROGENS ON CVD IN DIABETIC WOMEN}

Considerable attention has been focused on androgens in diabetic women because of their increased risk for CVD. Postmenopausal women with diabetes have elevated 
levels of testosterone compared to non-diabetic controls. Stoney et al (79), in a study among 42 postmenopausal diabetic women compared with 42 normoglycemic women matched for age and BMI, reported that diabetics had significantly elevated levels of androgens. Another study of 126 postmenopausal diabetic patients with 7.8 years average duration of diabetes, matched for age and BMI with 126 healthy postmenopausal controls also demonstrated that diabetics have elevated levels of testosterone than controls (1.7+/-0.1 vs. 1.4+/-0.2 pmol/l; P<0.05) (80). Furthermore diabetic women have a higher ratio of testosterone/estradiol than controls (80). The association was also confirmed by a meta-analysis of 43 prospective and cross-sectional studies in which women with diabetes had higher testosterone (mean difference, $6.1 \mathrm{ng} / \mathrm{dL}$; 95\% CI, 2.3 to 10.1) and estradiol levels compared to age and BMI matched controls (81). Even among diabetic women with similar total fat mass, android adiposity, waist circumference and upper body fat as non diabetic controls, the same relationship is observed (85). Other studies of diabetic women matched with non-diabetics either on age and years since menopause (82), or regression adjustment for age, BMI and waist to hip ratio $(83,84)$ concluded that testosterone levels are indeed elevated among diabetics.

It has been proposed that the ovaries of Women with diabetes have a reduced ability to convert androgens to estrogens due to a reduction of aromatase activity (94). Although the source of the excess androgens in diabetic women has not been studied in detail, results in women with PCOS, who are also more likely to be obese and insulin resistant $(91,92)$, and to develop diabetes suggest an ovarian source of androgens although increased adrenal steroid production might also contribute in part to the higher androgen levels reported in diabetic women (93). 
Table 2.2: Studies of endogenous testosterone and risk of cardiovascular disease (CVD) outcomes in postmenopausal women

\begin{tabular}{|c|c|c|c|c|c|c|c|c|}
\hline \multirow[t]{2}{*}{ Study } & \multirow{2}{*}{$\begin{array}{l}\text { Study } \\
\text { design }\end{array}$} & \multirow[t]{2}{*}{$\mathrm{N}$} & \multirow{2}{*}{$\begin{array}{l}\text { Mean } \\
\text { age }\end{array}$} & \multirow{2}{*}{$\begin{array}{c}\text { Mean } \\
\text { BMI }\end{array}$} & \multicolumn{3}{|c|}{ Risk estimate } & \multirow[t]{2}{*}{ Outcome } \\
\hline & & & & & Total Testosterone & Free Testosterone & Bioavailable $\mathrm{T}$ & \\
\hline $\begin{array}{l}\text { Barrett-Connor et al } 1995 \\
(261)\end{array}$ & $\mathrm{CS}$ & 651 & 66.7 & - & HR:1.00 (0.99-1.01) & & RR:0.98 (0.95-1.02) & CVD death \\
\hline Rexrode et al $2003^{(263)}$ & $\mathrm{NCC}$ & 400 & 63.1 & 26.4 & $\begin{array}{c}\text { Q4 vs.Q1 } \\
\text { OR:1.16 }(0.48-2.78)\end{array}$ & & & $\begin{array}{l}\text { nonfatal MI, } \\
\text { PCI, CABG, } \\
\text { nonfatal stroke, or } \\
\text { stroke death, }\end{array}$ \\
\hline Braunstein et al $2008^{(264)}$ & $\mathrm{C}$ & 284 & 64.8 & 30.0 & OR:1.02 (1.00-1.04) & $\begin{array}{c}\text { OR:1.13 (1.20- } \\
1.25)\end{array}$ & & $\begin{array}{l}\text { CAD }(\geq 50 \% \\
\text { Stenosis })\end{array}$ \\
\hline Patel et al $2009^{(265)}$ & $\mathrm{C}$ & 344 & 74.4 & 26.9 & $\begin{array}{c}\text { Q4 vs. Q2 } \\
\text { OR: } 2.95(1.2-7.3)\end{array}$ & $\begin{array}{c}\text { Q4 vs. Q2 } \\
\text { OR:1.41 (0.6-3.2) }\end{array}$ & & $\begin{array}{l}\text { Prevalent CHD } \\
\text { (angina, MI, } \\
\text { coronary } \\
\text { angioplasty, } \\
\text { or CABG }\end{array}$ \\
\hline Sievers et al $2010^{(262)}$ & $\mathrm{CS}$ & 2914 & 58.0 & 26.7 & $\begin{array}{c}\text { Q5 vs. Q1 } \\
\text { HR:0.64 (0.38-1.07) } \\
\text { Q2-Q5 vs. Q1 } \\
\text { HR:0.68 }(0.48-0.97)\end{array}$ & & & $\begin{array}{l}\text { angina, MI, } \\
\text { PCI, CABG, } \\
\text { stroke, transient } \\
\text { ischemic attack, } \\
\text { CV death. }\end{array}$ \\
\hline Laughlin et al $2010^{(266)}$ & $\mathrm{CS}$ & 639 & 73.8 & 24.3 & $\begin{array}{c}\text { Q1 vs. Q2-Q5 } \\
\text { HR:1.72 (1.15-2.57) }\end{array}$ & & $\begin{array}{c}\text { Q5 vs. Q3 } \\
\text { HR:1.84 (1.06-3.19) } \\
\text { Q1 vs. Q3 } \\
1.93(1.09-3.43)\end{array}$ & $\begin{array}{l}\text { coronary artery } \\
\text { revascularization, } \\
\text { nonfatal MI, } \\
\text { or fatal MI. }\end{array}$ \\
\hline Wehr et al 2011 & CS & 875 & 67.3 & 26.9 & HR:1.03 (0.78-1.37) & HR:1.01(0.89-1.15) & & CVD death \\
\hline
\end{tabular}

T: Testosterone, C: Cross-sectional study, CS: Cohort study, NCC: Nested case control study, OR: odds ratio, HR: Hazard ratio, CAD: coronary artery disease, MI: myocardial infarction, PCI: Percutaneous coronary intervention, CABG: Coronary Artery Bypass Grafting 
Lower sex-hormone binding globulin (SHBG) levels in diabetics further increase the non-SHBG (bioavailable) testosterone in the circulatory system, and amplify the effect of testosterone independent of overall adiposity (body mass index) and upper body adiposity (as measured by the ratio of waist to hip circumferences) on CVD risk (87). Although some studies have found low levels of SHBG and high androgenicity to be associated with dyslipidemia and increased carotid artery intima-media thickness which in turn is associated with an adverse CVD risk factor profile in postmenopausal women (88-90), evidence for an association with incident hard cardiovascular endpoints especially among diabetic women in longitudinal studies is lacking. A case control-study of 120 (40 cases and 80 controls) postmenopausal diabetic women from the Wisconsin Epidemiologic Study of Diabetic Retinopathy reported no association between endogenous testosterone and 5 year risk of ischemic heart disease mortality (268). This same study reported that low levels of dehydroepiandrosterone sulfate predicted ischemic heart disease mortality. However this study included women with type-1 and type- 2 diabetes, and no stratification was made for type of diabetes in the analysis. Wehr et al (267), followed 875 postmenopausal women for a median of 7.7 years and found no association between total testosterone or free testosterone levels with either CVD or all cause mortality. Interestingly, in a subsample of 302 women with diabetes, comparing women in the fourth quartile to the first quartile, free testosterone but not total testosterone predicted all-cause (HR: $0.38,95 \%$ CI: 0.08-0.90) and cardiovascular mortality (HR: $0.28,95 \% \mathrm{CI}: 0.08-0.90$ ). This association was not linear thought, as there was no significant association of one standard deviation increase in free testosterone with all-cause mortality or cardiovascular mortality. 
Low androgen levels among postmenopausal women may be caused by illness or weight loss which also has adverse impact on mortality or health (267). Besides this, the external validity of the study is limited since it was not population based but was instead conducted among women referred for coronary angiography, therefore results may not be applicable to women with low cardiovascular risk.

To resolve the ambiguity in the scientific literature as to whether low or high levels of androgens are associated with CVD events in diabetic women may require assessing levels of androgens among postmenopausal women with and without intact ovaries since the latter are known to have relatively low levels of androgens. If low levels of androgens somehow predispose to CVD among diabetics, then a decreased risk should be found among diabetic women with intact ovaries compared to diabetic women with oophorectomy. On the other hand, if elevated levels of androgens contribute to increased CVD risk in diabetics, then diabetic women with oophorectomy will have a relatively reduced risk compared to diabetic women with intact ovaries.

Further, if accumulation of visceral fat due to estrogen deficiency is an important predictor of CVD risk and androgen levels then the removal of the ovaries at an early age may have a more adverse effect on CVD risk than if this procedure is performed after the time of natural menopause. An alternative possibility is that some women whose ovaries are removed at an early age are insulin resistant and anovulatory (95), representing a group of women with increased risk of CVD.

\section{Summary}

The relative importance of testosterone to women's cardiovascular health remains a matter of great speculations. While some studies have found no statistical relationship 
between testosterone and CVD events in women, other which found a significant association reported either a linear, J- or U-shaped relationship. Although much progress has been made in the prevention of cardiovascular disease, it remains the leading cause of morbidity and mortality in women. The presentation, diagnosis and treatment of CVD differ between women and men leading to gender related disparities in outcomes. Moreover, the impact of type 2 Diabetes on CVD differs substantially by gender. With the diagnosis of diabetes, the relative risk of CVD is greater in women than men, with women having more adverse outcomes than men. Although, it is controversial that menopause increases the risk of CVD in women, this transitional period is associated with several changes in both lipid profiles and body composition which is thought to be influenced by sex hormones. During menopause, women experience a significant decline in estrogen levels compared to androgens, which creates a more androgenic profile. These excess androgens are suggested to modulate endothelial function, resulting in a more deleterious effect on the coronary vasculature thereby predisposing postmenopausal women to an increased risk of cardiovascular diseases. Evidently, diabetic women have elevated levels of androgens and if androgens have adverse effect on women's cardiovascular health, then this might explain why diabetic women are at greater risk of CHD events. Despite some studies indicating that BSO increases cardiovascular disease risk, studying women with oophorectomy remains a good model to investigate and understand the effect of hyperandrogenemia and cardiovascular outcomes among postmenopausal diabetic women. 


\section{CHAPTER THREE}

\section{RESEARCH DESIGN AND METHODS}

\section{NATIONAL HEALTH AND EXAMINATION SURVEY (1988-2010) Description and source of study population}

The National Health and Nutrition Examination Survey (NHANES) is a national survey conducted by the National Center for Health Statistics to assess the health and nutritional status of children and adults residing in the United States. Its aim is to monitor health trends and provide national estimates of certain health risk factors and outcomes. Authorized by the National Health Survey Act of 1956 passed by the $84^{\text {th }}$ Congress, which stipulated that health information be continuously collected to monitor the health status of the civilian non-institutionalized population, this health survey has been conducted since 1960 (269). The first three National Health Examination Surveys (NHES I, II, and III) conducted from 1960 to 1970 did not collect any information on nutritional status. All subsequent surveys since that time have included a nutritional and an environmental health component. As such, the name of the survey was changed to the National Health and Nutrition Examination Survey and data collection was also broadened to include ages 1-74 years. NHANES I was conducted from 1971 to 1975; adult participants aged 25 years and older were subsequently followed longitudinally until 1992 in the NHANES I Epidemiological Follow-up Survey (NHEFS). 
NHANES II was conducted between 1976 and 1980, and NHANES III between 1988 and 1994 (269). After this time, the periodic collection of data was discontinued, and since 1999 data have been collected annually. Due to disclosure issues, continuous survey data are released in two-year increments. Mortality data for persons who were 17 years or older at the time of interview in the various surveys conducted between 1971 and 2004 were obtained by the NCHS using a probabilistic match of participants information to the National Death Index records (269).

\section{Sampling procedure}

Both NHANES III and the continuous NHANES surveys employ a complex multistage design to obtain a probability sample of the civilian, non-institutionalized U.S. population. Certain subpopulations such as adolescents aged 12 to 19 , African Americans, Mexican Americans, low income White Americans, and adults over the age of 60 are oversampled to increase reliability and precision of estimates of health indicators for these subgroups.

The complex multistage sampling begins with primary sampling units (PSU) which includes single counties or groups of contiguous counties with probability proportional to a measure of size (269). The PSUs are then divided into segments-usually city blocks-and households within each segment are randomly sampled, with individuals in those households chosen within designated age-sex-race/ethnicity screening subdomains (269). On average 1.6 persons are selected per household (269). The Survey consisted of a detailed in-person home interviewer-administered questionnaire using Computer-Assisted Personal Interview (CAPI) technology, physical examination and 
specimen collection performed in a set of specially-designed and equipped Mobile Examination Centers (MEC).(269). All adult participants provided written informed consent and all procedures and surveys were approved by the National Center for Health Statistics Institutional/Ethics Review Board. During the period of 1988 to 2010, the response rate for the home interview ranged from $78.4 \%$ to $86.0 \%$ while that for the medical examination ranged from $75.4 \%$ to $80.0 \%$ (270).

\section{Study eligibility criteria}

\section{Inclusion criteria}

Analyses for the current study were restricted to NHANES III since it was the first wave to ask reproductive health questions pertaining to oophorectomy status, and also NHANES 1999-2008. While NHANES 1999-2008 provided cross-sectional information for assessing cardiovascular disease in diabetic women with oophorectomy, NHANES III allowed the evaluation of the prospective association of the effect of oophorectomy in diabetic and cardiovascular mortality. NHANES III was linked with mortality follow-up data from 1988 through to December 31, 2006. Mortality was ascertained based on National Death Index death certificate records. Previous versions of the International Classification of Diseases (ICD) codes were recoded into comparable ICD-10 codes based on underlying cause of death.

Of the 39,695 persons selected to participate in NHANES III, 33,994 (86\%) were interviewed in their home with 30,818 (78\%) of them receiving a clinical examination. Female participants who were $\geq 40$ years old and not pregnant $(n=6,050)$ were eligible for this study. For the six waves of NHANES data (1999-2010), 15,644 female 
participants aged 20 years or older who received a clinical examination were eligible for this study.

\section{Exclusion criteria}

From the 6,050 women in NHANES III eligible for the study, the following exclusions were made, in order: 1,984 pre- and perimenopausal, 356 with partial or unilateral oophorectomy, 384 with missing age at menopause, 18 with missing values for either height or weight or both, 100 with missing values for important covariates, and 2 women with missing mortality status. In addition, women who were underweight $(\mathrm{n}=82)$ based on BMI were excluded due to the small number, yielding an analytic sample comprising of 3,224 postmenopausal women. In NHANES 1999-2010, analyses were restricted to 7,139 women who were postmenopausal. Of this number, 359 with partial or unilateral oophorectomy, and 405 women with unknown or missing age at menopause were excluded, resulting in a sample size of 6,375 for the cross-sectional analysis.

\section{Definition of exposure variables}

\section{Diabetes}

Both fasting and 2 hour oral post-glucose (using $75 \mathrm{~g}$ oral dose in adults) concentrations were determined by an enzyme hexokinase method. Diabetes was defined as a fasting (at least 8 hours) plasma glucose of $\geq 126 \mathrm{mg} / \mathrm{dl}$ or $<126 \mathrm{mg} / \mathrm{dl}$ with a 2-hour value of $\geq 11.1 \mathrm{mmol} / \mathrm{L}$ ( $200 \mathrm{mg} / \mathrm{dL})$ in accordance to the Expert Commission on Diagnosis and Classification of Diabetes recommendations for epidemiologic studies (271). In addition, current use of an antihyperglycemic medication (including insulin) or 
a self-reported medical history of diabetes was also included in the definition of prevalent diabetes.

\section{Oophorectomy}

The detailed home interview questionnaire ascertained both oophorectomy status and age at surgery. Oophorectomy status was ascertained through questions such as "have you had one or both of your ovaries removed", "were only one or both ovaries removed" and "how old were you when ovaries were removed" Women who answered "both ovaries were removed" were considered to have bilateral oophorectomy. Participants were not asked about the indication for oophorectomy.

\section{Definition of outcome variables}

Prevalent cardiovascular disease (CVD) was defined as a self-report of physician diagnosis of angina pectoris, stroke and myocardial infarction. In NHANES III, angina was assessed using the WHO Rose angina questionnaire (272). For prospective analyses, deaths were classified as incident CVD mortality when the underlying or primary cause of mortality on the death certificate was coded with ICD 10 codes (I00 - I78). A dichotomous variable with categories for CVD mortality and no-CVD mortality was created.

\section{Definition of relevant covariates}

\section{Sociodemographic}

Information about age, race/ethnicity, and educational status was self-reported. Age was specified as continuous. Race/ethnicity was defined as Non-Hispanic White, 
Non-Hispanic Black, Mexican American and Other. Educational status was reported as years of education, and further categorized into either college degree or higher and less than a college degree. Socio-economic status was assessed using the poverty income ratio. This is the ratio of a family's income to the poverty threshold defined by the U.S. Census Bureau appropriate to the family's composition (273). A cutoff point of less than 1.00 means living below the federal poverty level while a cutoff point of 1.00 or more means living at or above the federal poverty level.

\section{Anthropometry}

Body mass index (BMI) was calculated as weight in Kilograms divided by the square of height in meters, after which it was categorized into underweight (less than 18.5), normal weight (18.5 to 24.9 ), overweight (25 to 29.9 ) and obese as 30.0 or greater, without any modification/adjustment being made for any racial/ethnic group. Waist circumference was measured to the nearest $0.1 \mathrm{~cm}$ at the level of the iliac crest. Women who had a waist circumference of $>88 \mathrm{~cm}$ were considered to have abdominal obesity. Hip circumference was measured at the level of the maximum posterior protrusion of the buttocks. Waist-to-hip ratio (WHR) was calculated by dividing waist circumference by hip circumference.

\section{Health behavioral/lifestyle factors}

A subject was be said to be a "never smoker" if she reported smoking fewer than one hundred cigarettes over her entire life. "Former smoker" was defined as reporting smoking at least one hundred cigarettes over a participant's lifetime, but answered "no" 
when asked if she smoked at the present time. Finally participants were categorized as "Current smoker" if they reported smoking at least one hundred cigarettes over their lifetime and answered "yes" when asked if they smokes at the present time.

For physical activity, participants were asked about the frequency with which they had participated in the following activities during the preceding month: walking a mile without stopping, jogging or running, swimming, regular dancing, aerobic exercise or

aerobic dancing, riding a regular bicycle or exercise bicycle, calisthenics, garden or yard work, and weight lifting. Only frequency of events and not duration were asked. The frequency of physical activity in the previous month was divided into 3 levels based on their distribution: low if frequency of activity was less than 5, medium (5-28 times) and high (29 or more times).

\section{Health history and conditions}

During the home interview, participants were asked whether any family member had a heart attack before age 50. A family member was defined as an immediate blood relations living or deceased, which would include grandparents, parents, brothers and sisters. Participants who responded "yes" were considered to have a family history of heart attack before age 50. Additionally, participants were asked if they had been informed by a physician or were told to be diagnosed with cancer. A history of female cancer was defined as having a physician diagnosis of breast, uterus or ovarian cancer.

Blood pressure was measured by trained staff using a mercury sphygmomanometer with an appropriately sized arm cuffs. Participants had their blood pressure measured while seated. A participant was said to have hypertension if there was 
a systolic blood pressure above $140 \mathrm{~mm} \mathrm{Hg}$, diastolic blood pressure of above $90 \mathrm{~mm}$ $\mathrm{Hg}$, a physician diagnosis of hypertension, or current medication use for elevated blood pressure.

Non-fasting serum levels of HDL-cholesterol and C-reactive protein were measured using the Roche/Boehringer-Mannheim Diagnostics direct HDL method and the latex-enhanced nephelometry on a Behring Nephelometer respectively (274).

In NHANES III, fasting measures of triglycerides, plasma glucose, and insulin were obtained in a subsample of participants $(\sim 50 \%)$ who attended the morning examination. Hypercholesterolemia was defined as a serum total cholesterol level $\geq 240$ $\mathrm{mg} / \mathrm{dl}$.

\section{Reproductive heath}

Questions on reproductive health were collected by a trained interviewer. Participants with no history of reproductive surgery were classified as postmenopausal if they reported not having had a menstrual period during the previous 12 months; consistent with WHO criteria (275). Additionally, women with no history of hysterectomy or oophorectomy who were current users of postmenopausal hormone therapy (HT) and were $\geq 51$ years were classified as postmenopausal. HT use was also obtained by asking the following questions: "have you ever taken estrogen or female hormone pills by mouth other than oral contraceptives", "have you ever used female hormones in the form of vaginal creams, suppository, injections or skin patches”.

Duration of HT was also obtained. Current HT users were women who answered "yes" to using female hormones in the form of pills, vaginal cream, suppository, injections or 
patches placed on the skin, and were currently using them. Former users were those who had used female hormones after menopause but were not currently using them. Never users of HT were those who answered "no" to any of the above questions. Oral contraceptive use was assessed by asking "have you ever taken birth control pills for any reason" with a response of "Yes" indicative of ever use. Women were also asked about their age at menarche, and this was reported in whole years. Finally, participants were asked about the number of pregnancies resulting in live births. Based on response, parity was categorized as none, 1-2 live births, 3-4 live births and $\geq 5$ live births.

\section{Power Calculations}

A previous study which examined the incidence of CVD in diabetic men and women reported that among diabetic women aged 50-79 and free of heart disease, approximately $37 \%$ developed CVD at the end of 10 years of follow-up (276). Likewise, another study examined the impact of diabetes on CHD among 121,046 women in the Nurses' Health Study aged 30 to 55 years, and followed from 1976 to 1996, reported that $40 \%$ of diabetic women developed coronary heart disease (10). A power calculation for the current study was made based on these results (277).

For NHANES III which had an accrual interval of 6 years, and additional followup of approximately 12 years for mortality, if the true relative risk of CVD in diabetic women with a history of $\mathrm{BSO}(\mathrm{N}=211)$ relative to diabetics without $\mathrm{BSO}(\mathrm{N}=812)$ is 0.74, we will be able to reject the null hypothesis that the exposed and unexposed survival curves are equal with a power of $0.84(\alpha=0.05)(277)$. For the cross sectional association in NHANES 1999-2010, the sample size was sufficient to detect an odds ratio 
of 0.76 among 369 diabetic women with $\mathrm{BSO}$ and 1,034 diabetic women without BSO, based on prior data indicating that the average proportion of diabetics with CVD is $38 \%$, resulting in a power of more than $85 \%(\alpha=0.05)(278)$.

\section{THE STUDY OF OSTEOPOROTIC FRACTURES (SOF)}

\section{Study population and sampling}

The study of osteoporotic fractures (SOF) is a multi-center prospective observational study of women age 65 or older with clinical sites at University of Maryland, Baltimore; University of Minnesota, Minneapolis; Kaiser-Permanente Center for Health Research in Portland, OR; and the University of Pittsburgh, Pittsburgh (14, 279). It was funded by the National Institute of Arthritis and Musculoskeletal and Skin Diseases to describe the risk factors for osteoporotic fractures and falls in postmenopausal women. However, its aims have grown to include factors that influence successful aging. Originally, 9704 women who were predominantly non-Hispanic whites were recruited from September 1986 to October 1988 through population-based listings, and enrolled into the study.

Women were recruited from several sources. In Minneapolis, women were identified from Group Health Incorporated, a large health maintenance organization; list of Minnesota residents produced for the Hypertension Detection and Follow-up study as well as the Systolic Hypertension in the Elderly study; and jury-selection lists for Hennepin County (280). In Pennsylvania, women were identified from the 1985 voter's registration list for the Monongahela Valley (280). In Portland, women were identified from membership lists for the Kaiser-Permanente Health Plan, which is also a large 
Health Maintenance Organization (280). Finally, in Baltimore women were selected from lists of holders of driver's license and identification cards within Baltimore County (280).

Participants received a letter and a brochure inviting them to join the study. Data were collected from all four clinical sites using a common protocol and detailed manuals of operation. At the baseline clinic visit, physical examination, health status questionnaires, dietary history, medical history, lateral lumbar radiographs, and blood specimens were collected. Study participants were contacted every four months by postcard or telephone to ascertain the occurrence of falls, fractures and changes in residential address. Participants returned every two years for clinical visits. To date, rates of follow-up have exceeded $98 \%$ (281).

Since African American women are known to have a low incidence of hip fracture (which was the main outcome), they were excluded from the initial recruitment. Additionally, women were excluded if they had a history of bilateral hip replacement or were unable to walk without assistance (282). From 1996 to 1998, the original cohort was enhanced by the addition of 662 African American women recruited at the Maryland and Pennsylvania clinical centers, yielding a total cohort of 10,366 women (281). All participants provided written informed consent and human subjects research approval was obtained by each clinical center's institutional review board.

\section{Eligibility criteria}

\section{Inclusion criteria}

Incident cardiovascular disease related mortality was not available for the African American cohort. Therefore, two analytic cohorts were created. The first, which included 
10,366 women, was to assess the incidence of CVD disease while the second was comprised of the original cohort of 9,704 women enrolled between 1986 and 1988 was used to evaluate CVD deaths. Prevalent cardiovascular disease was evaluated at the second visit; therefore, this time was used as baseline for studying incident nonfatal CVD.

\section{Exclusion criteria}

Women were excluded from the analytic cohort if they had missing or unknown age at last menstrual period $(\mathrm{N}=2,004)$. Furthermore, women who had unilateral or missing values for oophorectomy status $(\mathrm{N}=412)$ were also excluded resulting in a cohort of 7,950. Analyses of time to CVD deaths was restricted to the Caucasian cohort $(n=7540)$ since cardiovascular disease related mortality was not ascertained in the African American cohort.

\section{Definition of exposure variables}

\section{Diabetes}

History of diabetes was determined by means of a questionnaire at baseline. A diagnosis of diabetes was established by a positive response to the question "has a doctor ever told you that you had diabetes or sugar diabetes". Women identified to be diabetic were further asked about age at diagnosis and current use of insulin.

\section{Oophorectomy}

By means of a questionnaire, women were asked "Have you ever had an ovary removed". Those who responded "yes" were further asked if one or both ovaries were 
removed. Women who had both ovaries removed were defined to have bilateral oophorectomy. Age at which this surgical procedure was performed was also ascertained.

\section{Definition of outcome variables}

At every subsequent clinic visit after the baseline assessment, participants were asked about incident physician diagnosed cardiovascular disease, which was defined as angina, myocardial infarction, stroke and other heart diseases. During the twenty years of follow-up, the coordinating center obtained copies of the original death certificates of all subjects who died and cause-specific mortality was coded according to the International Classification of Diseases, Ninth Revision Clinical Modification. Cause of death was physician-adjudicated and based upon death certificates and discharge summaries. Cardiovascular mortality was defined using ICD-9 Codes $(402,404,410-414,426-445)$ which represented mortality from atherosclerosis, stroke, coronary heart disease or other heart diseases.

\section{Definition of relevant covariates}

\section{Sociodemographic}

Self-reported data on age, race/ethnicity, highest grade or years of education completed, and marital status were obtained. Age was defined as continuous. Education was categorized into either a high school education or less, or otherwise. With regards to race/ethnicity, all participants identified themselves as non-Hispanic whites. No information about household or annual income was ascertained at baseline. 


\section{Anthropometry}

Body weight was obtained using a beam balance scale by weighing women while they wore indoor clothing without shoes. Height was measured using a portable wall mounted Harpenden stadiometer. Body mass index (BMI) was calculated as weight in kilograms divided by the square of height in meters, after which it was categorized into underweight (less than 18.5), normal weight (18.5 to 24.9), overweight (25 to 29.9) and obese as 30.0 or greater. Waist circumference was measured with a steel measuring tape at the midpoint between the highest point of the iliac crest and the lowest part of the costal margin in the midaxillary line. Hip circumference was measured at the level of maximum protrusion of the buttocks to the nearest $0.1 \mathrm{~cm}$. Waist-to-hip ratio was calculated by dividing waist circumference by hip circumference

\section{Health behavioral/lifestyle factors}

Smoking status was assessed using self-administered questionnaires. Smoking status was classified as never, former and current. The average number of cigarettes smoked per day as well as lifetime pack year smoked was also ascertained. Recreational and sports activities from the previous 12 months were recorded using a modified version of the Harvard Alumni Questionnaire and converted into weekly caloric expenditure by intensity level. The sum of kilocalories from high, medium and low intensity activities, stair climbing and walking was used to estimate total kilocalories per week expenditure during the previous year. Physical activity was defined as a continuous variable of total kilocalories expended per week. 


\section{Health history and conditions}

A personal history of cardiovascular disease was assessed by asking, "has a doctor ever told you that you have or had a stroke, a blood clot in the brain, or bleeding in the brain" (at baseline) and "has a doctor told you that you had a heart attack, coronary or myocardial infarction" (at second clinic visit). Women who responded "yes" were identified as having a physician diagnosis of cardiovascular disease. Questions were also asked to identify a personal history of angina, and congestive heart failure. A systolic blood pressure reading of $140 \mathrm{mmHg}$ and or a diastolic blood pressure of above $90 \mathrm{~mm} \mathrm{Hg}$ was used for the diagnosis of hypertension. Hypercholesterolemia was defined as a serum total cholesterol level $\geq 240 \mathrm{mg} / \mathrm{dl}$.

\section{Biochemical Analyses}

Blood samples were drawn from all participants according to the protocol of the Study of Osteoporotic Fractures. Blood was drawn between the hours of 8:00 a.m. and 2:00 p.m. Participants were not required to fast but were instructed to avoid fatty foods to minimize lipemia on the morning of the examination, which may interfere with assays. Blood was drawn after the participant had been seated for at least 10 minutes and was immediately frozen to $-20^{\circ} \mathrm{C}$ for up to 2 weeks and stored in liquid nitrogen at $-190^{\circ} \mathrm{C}$ until assays were done (283-285). Total cholesterol, triglyceride, high-density lipoprotein cholesterol, low-density lipoprotein cholesterol and sex hormones were measured in a subsample of participants. Sex hormone measurements were restricted to women not taking postmenopausal hormone therapy. 
Total testosterone was measured by radioimmunoassay after extraction and aluminum oxide column chromatography, with an interassay coefficient of variation of 6.1 to 13.4 percent (283-285). Free testosterone was measured with an ammonium sulfate precipitation procedure with an interassay coefficient of variation of 10.7 to 15.5 percent (283-285). Calculation of free testosterone was adjusted for albumin concentration (coefficient of variation for intra-assay and total assay, 5\% and 5.4\%, respectively; sensitivity, $34.7 \mathrm{pmol} / \mathrm{L})(286)$. Estradiol and estrone were measured in serum by radioimmunoassay after extraction and separation by liquid chromatography. The interassay coefficient of variation for estradiol ranged from 8 percent to 12.5 percent, and from 6.2 percent to 7.0 percent for estrone (283-285). The testosterone-binding capacity of SHBG in serum was measured by means of a displacement technique (283-285).

All biochemical Analyses were carried out at Coming Nichols Institute (San Juan Capistrano, CA) and Endocrine Sciences Esoterix (Calabasas Hills, Calif.). In both laboratories, the intra-assay and interassay coefficients of variation for total testosterone ranged from $4 \%$ to $12 \%$ and $9 \%$ to $11 \%$ for the assays done by Coming Nichols and from $3 \%$ to $13 \%$ and $9 \%$ to $14 \%$, respectively for assays done by Endocrine Sciences Esoterix (283-285). Blood samples from a subsample of 51 postmenopausal women were selected to determine the stability of these hormones in serum by analyzing serum samples both at baseline and after 3.5 years of storage at $-190^{\circ} \mathrm{C}$. The correlations between the two testosterone measures was $\mathrm{r}=0.99(\mathrm{P}<0.001)(285)$.

\section{Reproductive heath}

Participants completed a self administered questionnaire that assessed their reproductive health. Women were asked about their age at menarche and if at age 25 
years periods were regular or irregular. Age at natural menopause was determined by asking "how old were you at the time of your last natural menstrual period". Hysterectomy status was also assessed, as well as type of hysterectomy (abdominal or vaginal) and age at hysterectomy. Women who reported a history of hysterectomy were further asked about the reasons why they underwent a hysterectomy (fibroids or bleeding, pelvic pain or internal scarring, dropping or bulging out of female organs, surgical repair for leakage of urine and other reasons). This was used to represent indication for oophorectomy among women who had hysterectomy concomitant with bilateral oophorectomy. Hormone therapy use, duration, indication, route of administration and years since stopping HT use were also assessed. Oral contraceptive use was evaluated by asking "have you ever taken birth control pills" with a response of "yes" indicative of ever use. The number of pregnancies and live births were also obtained from all participants.

\section{Power calculation}

The same assumption used in the NHANES III prospective study of the incidence of CVD in diabetic women was employed. Based on SOF study having an accrual interval of 2 years, and additional follow-up of approximately 18 years, and given that we have an exposed group of 124 women and 383 unexposed women, We will be able to detect true hazard ratios (relative risks) of failure for unexposed subjects relative to exposed subjects of 0.716 or 1.442 with probability (power) 0.8 . The Type I error probability associated with this test of the null hypothesis that the exposed and unexposed survival curves are equal is 0.05.(277). 


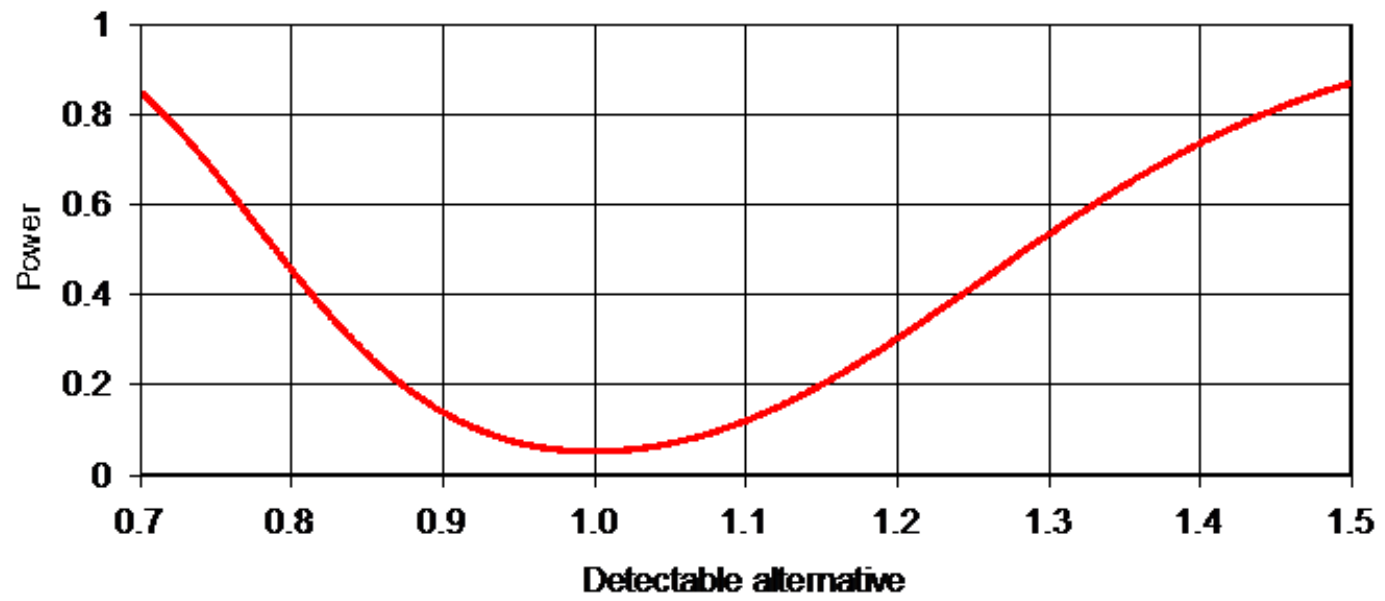

Figure 3.1 Detectable alternative relative risk based on a fixed sample size in the SOF

\section{Statistical Analysis}

\section{Descriptive statistics}

Descriptive statistics such as frequencies, percentages and means were calculated and used to describe the study participants according to diabetes and oophorectomy status. The four mutually exclusive groups were as follows: no history of diabetes or oophorectomy, history of diabetes without oophorectomy, no history of diabetes but with oophorectomy, and history of both diabetes and oophorectomy. For categorical variables, comparisons among groups were measured using the $\chi^{2}$ test while comparisons for continuous variables (namely sex hormones) were tested using analysis of variance (ANOVA) as well as analyses of Covariance with adjustment for age and BMI which was limited to women with diabetes. In instances when quantitative measures were skewed, they were normalized by natural log transformation and when normality was still not achieved by this procedure, a non-parametric test such as the Kruskal-Wallis test was employed. 


\section{Prevalent Cardiovascular disease}

The risk of cardiovascular disease given diabetes status, was assessed using logistic regression models using the SURVEYLOGISTIC procedure in SAS version 9.3 (SAS institute, Cary, NC) (287) which is suitable for analyzing NHANES data since it incorporates the complex sampling design and sampling weights. Failure to account for the clustered design, which incorporates differential probabilities of selection, introduces bias into the parameter estimates leading to overstating of statistical significance. Since the outcome (prevalent CVD) was dichotomous (that is the dependent variable takes on only two values: 1 for the probability of success and 0 for the probability of failure), logistic regression was appropriate to use to predict the risk of prevalent CVD conditioned on a set of categorical, discrete and continuous predictors. Adjusted odds ratios (OR) and 95\% confidence intervals (CIs) for risk factors were also calculated with alpha being set at 0.05 . No adjustments in $\mathrm{P}$ values were made for multiple comparisons and a two sided value was calculated. An a priori selection of confounders was made a priori based on the literature. Four models with progressive degrees of adjustments were employed in the analysis. Model 1 was adjusted for sociodemographic factors. Model 2 added reproductive factors to model 1 , while model 3 was further adjust for anthropometric measures and health behavioral/lifestyle factors. Finally, model 4 was adjusted for lipid measurements. These analyses were further limited to diabetics with women who had a history of diabetes being the referent as women with both diabetes and oophorectomy were compared to them. Additionally, the effect of age at oophorectomy on risk of CVD in diabetic women was further assessed. 


\section{Incident cardiovascular disease}

Participants in the SOF study who were alive and not lost to follow-up at the second visit were included in this analysis. Women with prevalent cardiovascular disease were excluded. Although incident cardiovascular disease was assessed, the date at which the event occurred was not provided in the public released data. In the light of this a discrete-time logistic model was employed (288).

This proportional odds model assumes that times are indeed discrete and not continuous. Briefly, this model proposes that the conditional probability $\mathrm{P}_{i t}$ that an individual $i$ has an event at time $t$, given that the event has not already occurred, is related to the covariates by the logistic regression equation (288).

$$
\log \left(\frac{P_{i t}}{1-P_{i t}}\right)=\alpha_{t}+\beta_{1} x_{i 1}+\ldots \ldots+\beta_{k} x_{i t k}
$$

Time takes on only positive integer values $(t=1,2,3 \ldots)$ which begins at some natural starting point $(\mathrm{t}=1)$. The observation continues until time $i t$, at which point either an event occurs or the observation is censored (288). The term $\alpha_{t}$ which represents a set of constants when unspecified is analogous to the baseline hazard in the proportional hazards model and can vary from one point to another. When the time intervals become progressively smaller the logistic model converges to the proportional hazards model (288). Using partial likelihood, the above logistic model implies that the ratio of the odds of any two individuals does not depend on time but may vary with covariates. Here the constant terms $\alpha_{\mathrm{t}}$ are treated as nuisance parameters and only estimates for $\beta_{\mathrm{k}}$ are calculated (288). On the other hand, the standard maximum likelihood estimation which can also handle large numbers of time dependent covariates does produce estimates for $\alpha_{t}$ 
together with the other parameters in the model. The partial likelihood method was chosen because if a participant who had an event was to miss a clinic visit, this event would not be recorded until the next visit. Since the basic assumption of the model is that the events really happened in the discrete time they were reported, a bias may be introduced when estimating the dependence of the hazard on time. However estimates of $\beta_{\mathrm{k}}$ from both the partial and maximum likelihood methods are always comparable (288).

The following confounders, which were chosen a priori, were controlled in multivariable models: age, race, education, body mass index, age at menopause, hypertension, hysterectomy status, parity, hormone therapy use, cigarette smoking, waistto-hip ratio and physical activity. Lipids were not measured in the African American cohort and therefore were not included in the multivariable model. Adjusted odds ratios and $95 \%$ confidence intervals for risk these factors were calculated. Further analyses were restricted to only diabetic women. All analyses met the minimum of 10 outcome events per predictor variable rule (289). The goodness-of-fit of all statistical models was determined by means of log-likelihood ratio test.

To assess the possibility of a dose-response association of age at natural menopause or bilateral oophorectomy on the incidence of cardiovascular disease among diabetics and nondiabetic women, logistic b-spline regression was implemented (290). Unlike categorization of a continuous variable which introduces cut-point bias and misclassification, splines (which are smooth continuous lines or curves) use the full range of data to estimate the shape of the association between a continuous variable and a dichotomous outcome $(290,291)$. Briefly, in this model the odds ratio for each value of a predictor $\mathrm{x}$, with respect to a reference value $\mathrm{x}_{\text {ref }}$ is calculated as 


$$
O R\left(x, x_{r e f}\right)=\exp \left(\sum_{i=1}^{n} \beta_{i}\left[s_{i}(x)-s\left(x_{r e f}\right)\right]\right)
$$

where $n$ is the number of degrees of freedom of the spline expansion, $\beta_{\mathrm{i}}$ is the coefficient of the $i^{\text {th }}$ spline basis function estimated by the logistic regression and $\mathrm{s}_{\mathrm{i}}(\mathrm{x})$ is the value of the $i^{\text {th }}$ spline basis function at $x$ (290). The $95 \%$ confidence intervals are also calculated based on asymptotic normality of the estimates. Akaike information criterion (AIC) was used to determine the goodness of fit of the model. The final model chosen was quadratic spline with three knots at the 25th, 50th and 75th percentiles of age at menopause. Knots represent thresholds in a risk factor where the probability of an event is assumed or expected to suddenly change $(290,291)$.

\section{Cardiovascular disease Mortality}

In the analysis of time to event, frequencies of incident CVD mortality were described. The Kaplan-Maier method, also known as the product-limit estimator, which is a non-parametric test, was used to estimate the risk of cardiovascular mortality during follow up in both NHANES III and the SOF study. Survival curves were then produced to graphically depict incident events with differences among groups assessed by means of the Log-Rank test. Although Kaplan-Maier methods cannot consider the effects of covariates on time to event, multivariable methods such as the semi-parametric Cox proportional hazards regression may be employed to overcome this limitation. Again, to assess the effect of complex sampling on the results, the SURVEYPHREG procedure in SAS was used for NHANES III while the non-survey PHREG procedure was employed for analysis using SOF data. 
The same method of adjustment for confounders in the logistic regression models were employed with adjusted hazard ratios and $95 \%$ confidence intervals calculated. However, because plasma lipids measures were measured only in a subsample of participants in the SOF study, they were not included in the multivariable models. A two tailed probability value less than 0.05 was considered statistically significant. In the SOF study analysis, the proportionality assumption was tested using cumulative sums of martingale residuals with a Kolmogorov-type supremum test and visually inspecting plots of Schoenfeld residuals versus time. However since these analyses cannot be accomplished with the survey procedure, the assumption of proportional hazards in NHANES III was assessed by introducing a time dependent coefficient in the Cox models. Subgroup analyses were conducted which were restricted to diabetics, investigating the moderating effect of oophorectomy on CVD mortality.

The time scale for all survival analyses was age in years. The advantages of using age instead of years since enrollment is that it has a more straight forward interpretation of mortality as it is free of the confounding effect of age which is intrinsically taken into account as a measure of survival time (292). Additionally, since no intervention was used in these prospective studies, modeling mortality rate as a function of time since enrollment is not interesting because we would be modeling mortality as a function of study duration, instead of the aging process (292). Furthermore, estimates of survival probabilities at every age in groups that are homogeneous according to the risk factors can be easily obtained (292). 


\section{CHAPTER FOUR}

\section{RESULTS}

\section{Characteristics of the study sample}

\section{The Third National Health and nutritional examination Survey, 1988-1994}

Demographic characteristics of the 3,324 postmenopausal women are presented in table 4.1, with descriptive statistics survey weighted. The average age of participants was 63.1 (median 62.3) years. Approximately, 83\% were non-Hispanic whites, while nonHispanic blacks made up $9.2 \%$ of women recruited for the study. Almost a third of survey participants $(27.3 \%)$ were diabetics, $46 \%$ had undergone a hysterectomy and $25 \%$ report a history of bilateral oophorectomy. A quarter of women (24.3\%) who reported a history of physician diagnosed diabetes were not on either insulin or oral hypoglycemic drugs. Among women with a hysterectomy, $46.3 \%$ reported ovarian conservation. Additionally, the prevalence of bilateral oophorectomy did not differ by diabetes status $(\mathrm{p}=0.303)$. The median age at bilateral oophorectomy was 42.3 years. Hypertension was reported in $42.65 \%$ of all participants while $11.2 \%$ of women were living $130 \%$ below the federal poverty level. With regards to hormone therapy use, $44.9 \%$ of women with oophorectomy were current users compared to $25.6 \%$ of women with hysterectomy and $9.2 \%$ of women with natural menopause. 
Table 4.1 Characteristics of study population, the third National Health and Nutrition Examination Survey (1988-1994)

\begin{tabular}{|c|c|c|c|c|c|c|}
\hline Characteristics & $\begin{array}{l}\text { No Diabetes/ } \\
\text { oophorectomy } \\
(\mathrm{N}=1695)\end{array}$ & $\begin{array}{c}\text { Diabetes w/o } \\
\text { oophorectomy } \\
(\mathrm{N}=812)\end{array}$ & $\begin{array}{l}\text { Oophorectomy } \\
\text { w/o diabetes } \\
(\mathrm{N}=506)\end{array}$ & $\begin{array}{l}\text { Diabetes and } \\
\text { oophorectomy } \\
(\mathrm{N}=211)\end{array}$ & $\mathrm{P}$ value & P value \\
\hline Age (years) & $63.9(62.8-64.9)$ & $64.0(62.8-65.2)$ & $61.3(59.6-63.0)$ & $59.9(57.4-62.3)$ & 0.001 & 0.003 \\
\hline Race/Ethnicity (\%) & & & & & 0.001 & 0.071 \\
\hline Non-Hispanic White & 83.5 & 76.3 & 86.3 & 84.7 & & \\
\hline Non-Hispanic Black & 7.8 & 12.6 & 9.3 & 10.8 & & \\
\hline Other & 8.7 & 11.2 & 4.4 & 4.5 & & \\
\hline College degree or higher & 29.7 & 24.9 & 30.3 & 21.0 & 0.155 & 0.436 \\
\hline PIR $\leq 130 \%$ poverty line $(\%)$ & 10.3 & 15.2 & 8.8 & 13.4 & 0.006 & 0.606 \\
\hline Smoking status (\%) & & & & & 0.664 & 0.815 \\
\hline former & 26.6 & 29.9 & 25.2 & 27.9 & & \\
\hline Current & 19.7 & 15.3 & 19.9 & 17.4 & & \\
\hline Age at menarche (years) & & & & & 0.001 & 0.420 \\
\hline$<12$ & 16.9 & 22.8 & 20.7 & 26.0 & & \\
\hline $12-14$ & 65.5 & 60.6 & 65.8 & 66.9 & & \\
\hline$>14$ & 17.6 & 16.7 & 13.5 & 7.1 & & \\
\hline Parity & & & & & 0.012 & 0.028 \\
\hline None & 16.3 & 12.7 & 21.9 & 15.7 & & \\
\hline 1 - 2 live births & 35.7 & 33.2 & 37.1 & 36.9 & & \\
\hline 3 - 4 live births & 31.5 & 30.3 & 28.0 & 31.4 & & \\
\hline$\geq 5$ live births & 16.5 & 23.7 & 13.0 & 16.0 & & \\
\hline Ever use of oral contraceptives (\%) & 31.7 & 26.4 & 36.5 & 29.1 & 0.113 & 0.611 \\
\hline
\end{tabular}




\begin{tabular}{|c|c|c|c|c|c|c|}
\hline Current Hormone therapy use (\%) & 14.7 & 11.6 & 45.9 & 42.7 & 0.001 & 0.001 \\
\hline Sedentary lifestyle $(\%)$ & 37.2 & 44.8 & 37.0 & 47.2 & 0.015 & 0.526 \\
\hline Waist Circumference $(\mathrm{cm})$ & $93.2(92.2-94.3)$ & $98.7(97.2-100.1)$ & $92.2(90.3-94.1)$ & $98.6(95.7-101.6)$ & 0.001 & 0.988 \\
\hline Waist to Hip Ratio & $0.90(0.89-0.91)$ & $0.94(0.93-0.95)$ & $0.89(0.88-0.90)$ & $0.91(0.89-0.93)$ & 0.001 & 0.017 \\
\hline $\operatorname{BMI}\left(\mathrm{kg} / \mathrm{m}^{2}\right)$ & & & & & 0.001 & 0.210 \\
\hline$<25.0$ & 38.7 & 27.6 & 44.4 & 21.9 & & \\
\hline $25.0-29.9$ & 34.8 & 34.7 & 30.7 & 31.5 & & \\
\hline$\geq 30.0$ & 26.5 & 37.6 & 25.0 & 46.6 & & \\
\hline HDL Cholesterol (mg/dl) & $56.3(55.2-57.5)$ & $51.7(49.8-53.7)$ & $58.3(55.9-60.7)$ & $52.7(50.1-55.4)$ & 0.001 & 0.529 \\
\hline Triglycerides (mg/dl) & $127.5(122.8-132.4)$ & $176.2(164.9-188.4)$ & $133.4(124.6-142.8)$ & $194.8(176.9-214.7)$ & 0.001 & 0.043 \\
\hline Total Cholesterol (mg/dl) & $227.3(224.0-230.1)$ & $239.8(235.1-244.5)$ & $231.3(225.4-237.2)$ & $241.6(233.9-249.2)$ & 0.001 & 0.698 \\
\hline C-reactive Protein (mg/dl) & $0.32(0.30-0.34)$ & $0.46(0.42-0.50)$ & $0.36(0.32-0.40)$ & $0.52(0.44-0.61)$ & 0.001 & 0.181 \\
\hline Glycohemoglobin (\%) & $5.41(5.37-5.46)$ & $6.49(6.29-6.69)$ & $5.34(5.29-5.38)$ & $6.36(6.11-6.61)$ & 0.001 & 0.438 \\
\hline White blood cell count & $6.52(6.38-6.66)$ & $7.25(7.05-7.45)$ & $6.69(6.49-6.90)$ & $7.46(7.09-7.85)$ & 0.001 & 0.302 \\
\hline Hypertension (\%) & 38.9 & 54.6 & 37.9 & 48.7 & 0.001 & 0.283 \\
\hline Family history of diabetes (\%) & 41.0 & 51.4 & 48.6 & 57.6 & 0.001 & 0.340 \\
\hline Family history of Heart Attack (\%) & 14.9 & 12.5 & 19.6 & 29.3 & 0.001 & 0.001 \\
\hline Angina pectoris $(\%)$ & 1.1 & 2.2 & 1.6 & 2.6 & 0.352 & 0.782 \\
\hline Stroke $(\%)$ & 3.4 & 7.8 & 5.0 & 8.6 & 0.001 & 0.740 \\
\hline Coronary Heart disease $(\%)$ & 5.2 & 10.0 & 7.4 & 14.5 & 0.001 & 0.190 \\
\hline Cardiovascular disease $(\%)$ & 7.5 & 16.0 & 10.6 & 20.0 & 0.001 & 0.264 \\
\hline
\end{tabular}

Values are survey weighted mean (95\% Confidence interval), or percentages for continuous or categorical variables, respectively

*P value comparing all four categories

${ }^{\text {II }} \mathrm{P}$ value for subgroup analyses restricted to only women with diabetes 
Diabetics compared to non-diabetics were more likely to be African Americans $(12.1 \%$ vs. $8.1 \%$ ), have lower socioeconomic status (14.7\% vs. $9.9 \%)$, no high school education (21.9\% vs. $17.3 \%)$, live a more sedentary lifestyle (45.5\% vs. $37.2 \%)$, obese (40\% vs. $26.2 \%$ ), hypertensive (53\% vs. $38.7 \%$ ), report earlier age at menarche (23.6\% vs. $17.8 \%$ ), and higher prevalence of stroke ( $8 \%$ vs. $3.8 \%$ ). Furthermore, in diabetic women, those with a history of oophorectomy were younger (59.9 vs. 64.0 years), more likely to have an earlier (<11 years) at menarche (26\% vs. $22.8 \%)$, and were more often current users of hormone therapy $(42.7 \%$ vs. $11.6 \%)$ and have a family history of myocardial infarction ( $28.4 \%$ vs. $12.2 \%$ ) compared to those with intact ovaries. On the other hand, diabetic women without oophorectomy had a higher WHR (0.94 vs. $0.91, \mathrm{p}=0.017)$ as measured by waist to hip ratio but no difference in waist circumference were found. While a greater proportion of diabetics had prevalent CHD and CVD, no differences were found with regards to oophorectomy status. Other baseline differences among diabetic women with and without oophorectomy did not attain statistical significance.

In non-diabetic, women who had an oophorectomy were more likely to be younger (61.3 vs. 63.9) African Americans (9.3\% vs. 7.8\%), current users of HT (45.9\% vs. 14.7\%) and reach menopause earlier (<45 years) $(60.5 \%$ vs. $30.5 \%)$,

Notably, women with diabetes and oophorectomy were more likely and to have a family history of myocardial infarction (29.3\% vs. $12.5 \%)$ compared to those diabetic women who did not undergo oophorectomy. A similar observation was also found in nondiabetics, where women with oophorectomy were more likely to have a family history of diabetes (48.6\% vs. $41.0 \%)$ and heart attack (19.6\% vs. $14.9 \%)$. 


\section{The National Health and Nutritional Examination Survey, 1999-2010}

Characteristics of NHANES 1999-2010 participants according to oophorectomy and diabetes status are illustrated in table 4.2. The survey-weighted average age of the 6,375 postmenopausal women sampled from the six waves of national survey data was 61.5 years. More than a third of participants (37.5\%) were obese, with $25.1 \%$ having sedentary lifestyles and $37.7 \%$ having metabolic syndrome (defined by the National Cholesterol Education Program's Adult Treatment Panel III criteria). Oophorectomy was performed in $27.5 \%$ of women, with the mean age at surgery reported as 43 years. Women with bilateral oophorectomy were more likely to be current smokers $(20.1 \%$ vs. $16.5 \%)$, to have had no college education (55.3\% vs. $49.4 \%)$, to have had an earlier age $(<50$ years $)$ at menopause ( $83 \%$ vs. $58.3 \%)$, and ever users of hormone therapy $(75.9 \%$ vs. $39.2 \%)$, hypertensive (56.4\% vs. $49.6 \%)$.

As in the earlier survey, nondiabetic women with oophorectomy were more likely to have a family history of myocardial infarction ( $21.6 \%$ vs. $15.9 \%)$, compared to women with intact ovaries. While no statistically significant effect of ovarian status was found with regards to lipid profiles, women with oophorectomy had higher levels of systemic inflammation markers namely C-reactive proteins ( 0.59 vs. $0.49 \mathrm{mg} / \mathrm{dl})$.

Approximately $17 \%$ of participants were diabetic and of this number $21.9 \%$ were on insulin therapy while $68.3 \%$ were currently taking anti-hyperglycemic agents. Diabetic women were more likely to have central adiposity as measured by waist circumference ( 107 vs. $96 \mathrm{~cm}, \mathrm{p}=0.001)$, be obese ( $55.9 \%$ vs. $33.8 \%)$, hypertensive (75.5\% vs. $46.6 \%)$, non-Hispanic blacks (16\% vs. $8.8 \%)$, have no college education (62.3\% vs. $48.7 \%)$, living at or below the federal poverty level (24.7\% vs. $17.4 \%)$, older 
age ( $\geq 55$ years) at menopause $(12.7 \%$ vs. $9.6 \%)$, never users of hormone therapy $(61.3 \%$ vs. $48.6 \%)$, higher levels of C-reactive proteins ( 0.65 vs. $0.49 \mathrm{mg} / \mathrm{dl}, \mathrm{p}=0.001)$ and a family history of myocardial infarction (17.3\% vs. $7.4 \%)$. Furthermore, diabetics had relatively worse lipid profiles. Converse to what has been reported in previous studies, non diabetics were more likely to be smokers compared to diabetics with (18.3 vs. $13.8 \%$ ) being current smokers.

The impact of age at oophorectomy in diabetic women was next analyzed (Table 4.3). A greater proportion of women with both diabetes and oophorectomy reported a history of cardiovascular disease compared to those with diabetes only. On the other hand, when oophorectomy was performed after age 45 the likelihood of CHD or CVD was reduced compared to diabetic women with no oophorectomy. Women with oophorectomy before age 45 also had a family history of heart attack more often than women with oophorectomy after age 45 or no oophorectomy (30.6\% vs. $20.9 \%$ vs. $18.2 \%)$

The same analyses of age at oophorectomy were repeated among nondiabetics (table 4.4). Women who had oophorectomy before age 45 years had a worse CVD risk profile than those who had oophorectomy at age 45 years or after. For instance, women with early oophorectomy were likely to be younger, African American, obese, current smokers and have no college education, sedentary lifestyles, lower HDL cholesterol levels and higher C-reactive protein levels. While a greater proportion of women with oophorectomy before age 45 years reported a family history of diabetes and heart attack, those who had oophorectomy at 45 years or after were found to have more CHD and CVD at baseline, an observation that maybe in part be driven by their older age status. 
Table 4.2 Characteristics of study population, National Health and Nutrition Examination Survey (1999-2010)

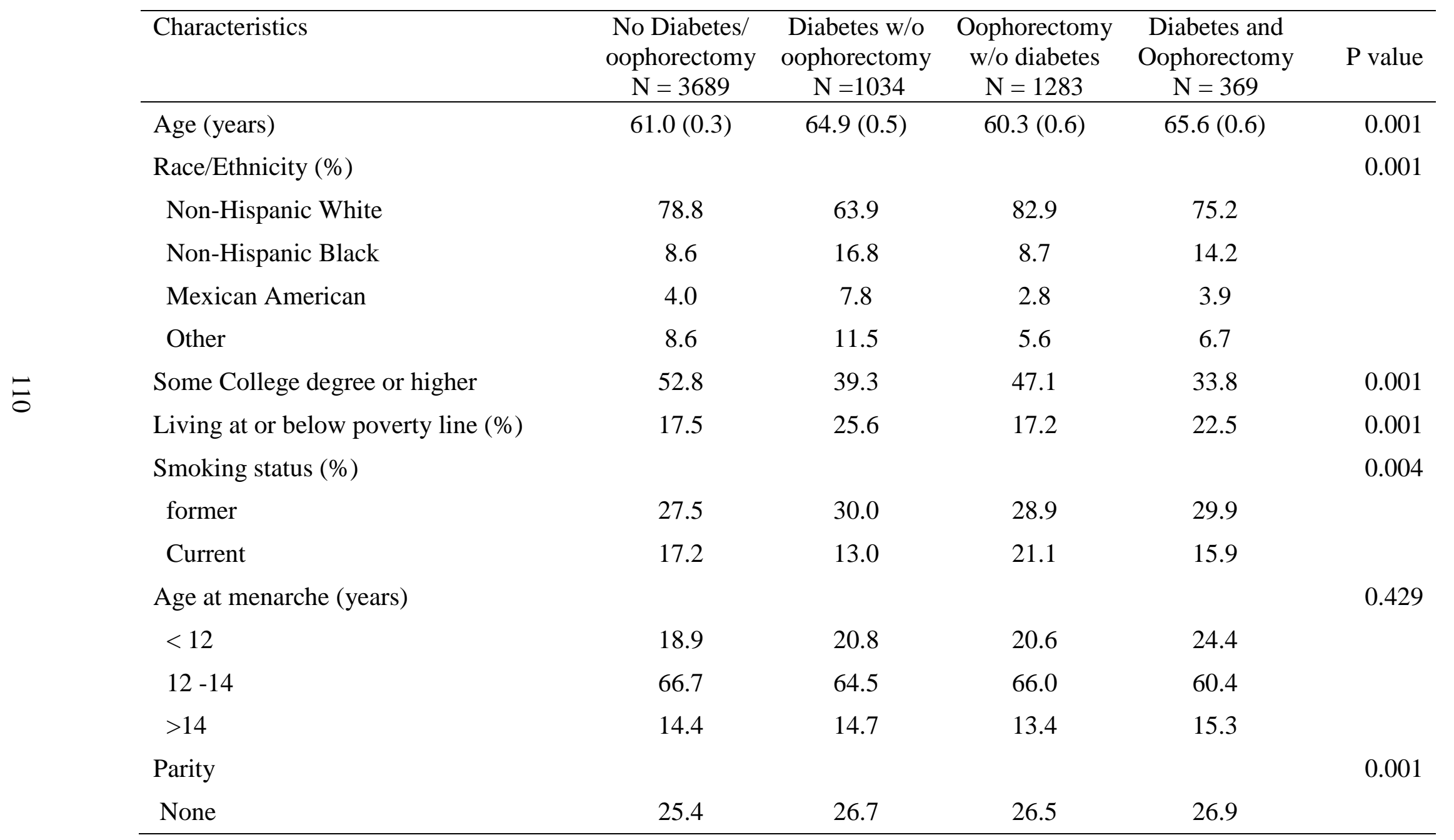




\begin{tabular}{lccccc}
\hline 1 - 2 live births & 37.2 & 28.3 & 35.5 & 31.5 & \\
3 - 4 live births & 27.3 & 27.7 & 30.3 & 28.6 & \\
$\geq 5$ live births & 10.1 & 17.3 & 7.8 & 13.0 & \\
Age at menopause & $45.7(0.2)$ & $46.4(0.4)$ & $40.9(0.3)$ & $41.5(0.6)$ & 0.001 \\
Ever use of Hormone therapy (\%) & 41.1 & 29.2 & 79.1 & 61.6 & 0.001 \\
Sedentary lifestyle (\%) & 24.6 & 25.7 & 26.6 & 23.8 & 0.549 \\
Waist Circumference (cm) & $95.5(0.3)$ & $106.8(0.7)$ & $95.9(0.5)$ & $107.7(0.9)$ & 0.001 \\
BMI (kg/m ${ }^{2}$ ) & & & & & 0.001 \\
$<25.0$ & 35.9 & 17.7 & 33.3 & 18.0 & \\
25.0 - 29.9 & 30.6 & 27.2 & 32.0 & 24.1 & \\
$\geq 30.0$ & 33.5 & 55.1 & 34.7 & 57.9 & \\
HDL Cholesterol (mg/dl) & $60.1(0.4)$ & $52.7(0.6)$ & $60.8(0.6)$ & $52.1(1.0)$ & 0.001 \\
Total Cholesterol (mg/dl) & $215.0(0.9)$ & $203.4(1.9)$ & $217.5(1.5)$ & $200(3.7)$ & 0.001 \\
C-reactive Protein (mg/dl) & $0.23(0.1-0.5)$ & $0.36(0.2-0.8)$ & $0.29(0.1-0.7)$ & $0.43(0.2-0.8)$ & 0.001 \\
Glycohemoglobin & $5.42(5.2-5.7)$ & $6.57(6.0-7.5)$ & $5.39(5.2-5.6)$ & $6.42(5.9-7.4)$ & 0.001 \\
Hypertension (\%) & 44.7 & 74.5 & 51.7 & 77.9 & 0.001 \\
Family history of diabetes (\%) & 35.5 & 57.9 & 39.6 & 57.2 & 0.001 \\
Family history of Heart Attack (\%) & 15.4 & 18.2 & 20.5 & 26.4 & 0.001 \\
Coronary Heart disease (\%) & 6.5 & 16.4 & 9.7 & 19.5 & 0.001 \\
Cardiovascular disease (\%) & 9.5 & 23.1 & 12.9 & 28.4 & 0.001 \\
\hline
\end{tabular}

Values are survey weighted mean (Standard error), median (interquartile range) or percentages for continuous or categorical variables, respectively 
Table 4.3 Characteristics of diabetic women according to age at oophorectomy, National Health and Nutrition Examination Survey (1999-2010)

\begin{tabular}{|c|c|c|c|c|}
\hline Characteristics & $\begin{array}{l}\text { No BSO } \\
N=1034\end{array}$ & $\begin{array}{c}\mathrm{BSO}<45 \\
\mathrm{~N}=207\end{array}$ & $\begin{array}{c}\mathrm{BSO} \geq 45 \\
\mathrm{~N}=162\end{array}$ & $P$ value \\
\hline Age (years) & $64.9(0.5)$ & $63.8(0.9)$ & $68.0(0.6)$ & 0.004 \\
\hline Race/Ethnicity (\%) & & & & 0.001 \\
\hline Non-Hispanic White & 63.9 & 74.0 & 76.9 & \\
\hline Non-Hispanic Black & 16.8 & 15.4 & 12.5 & \\
\hline Mexican American & 7.8 & 4.0 & 3.8 & \\
\hline Other & 11.5 & 6.6 & 6.9 & \\
\hline Some College degree or higher & 39.3 & 32.7 & 35.4 & 0.329 \\
\hline Living at or below poverty line (\%) & 25.6 & 23.7 & 20.9 & 0.522 \\
\hline Smoking status $(\%)$ & & & & 0.324 \\
\hline former & 30.0 & 28.9 & 31.3 & \\
\hline Current & 13.0 & 19.7 & 10.9 & \\
\hline Age at menarche (years) & & & & 0.111 \\
\hline$<12$ & 20.8 & 29.6 & 17.2 & \\
\hline $12-14$ & 64.5 & 54.5 & 68.4 & \\
\hline$>14$ & 14.7 & 15.9 & 14.4 & \\
\hline Age at menopause & $46.4(0.4)$ & $35.2(0.6)$ & $49.9(0.4)$ & 0.001 \\
\hline Ever use of Hormone therapy $(\%)$ & 29.2 & 64.5 & 57.6 & 0.001 \\
\hline Nulliparous & 26.7 & 30.2 & 22.4 & 0.442 \\
\hline Sedentary lifestyle (\%) & 25.7 & 28.8 & 17.1 & 0.057 \\
\hline Waist Circumference (cm) & $106.8(0.7)$ & $107.3(1.3)$ & $108.3(0.8)$ & 0.556 \\
\hline $\operatorname{BMI}\left(\mathrm{kg} / \mathrm{m}^{2}\right)$ & & & & 0.910 \\
\hline$<25.0$ & 17.7 & 17.9 & 18.0 & \\
\hline $25.0-29.9$ & 27.2 & 23.9 & 24.4 & \\
\hline$\geq 30.0$ & 55.1 & 58.1 & 57.6 & \\
\hline HDL Cholesterol (mg/dl) & $52.7(0.6)$ & $51.7(1.2)$ & $52.6(1.2)$ & 0.773 \\
\hline Total Cholesterol (mg/dl) & $203.4(1.9)$ & $202.0(4.7)$ & $197.5(3.0)$ & 0.408 \\
\hline C-reactive Protein (mg/dl) & $0.36(0.2-0.8)$ & $0.44(0.2-0.8)$ & $0.41(0.2-0.7)$ & 0.477 \\
\hline Glycohemoglobin & $6.57(6.0-7.5)$ & $6.46(5.9-7.5)$ & $6.39(5.8-7.2)$ & 0.591 \\
\hline Hypertension (\%) & 74.5 & 78.2 & 77.5 & 0.649 \\
\hline Family history of diabetes $(\%)$ & 57.9 & 55.1 & 60.0 & 0.707 \\
\hline Family history of Heart Attack (\%) & 18.2 & 30.6 & 20.9 & 0.010 \\
\hline Coronary Heart disease $(\%)$ & 16.4 & 25.4 & 11.6 & 0.007 \\
\hline Cardiovascular disease $(\%)$ & 23.1 & 35.5 & 18.9 & 0.001 \\
\hline
\end{tabular}

Values are survey weighted mean (Standard error), median (interquartile range) or percentages for continuous or categorical variables, respectively. BSO: Bilateral salpingo oophorectomy 
Table 4.4 Characteristics of non-diabetic women according to age at oophorectomy, National Health and Nutrition Examination Survey (1999-2010)

\begin{tabular}{|c|c|c|c|c|}
\hline Characteristics & $\begin{array}{c}\text { No } \\
\text { oophorectomy } \\
\mathrm{N}=3689 \\
\end{array}$ & $\begin{array}{c}\text { BSO }<45 \\
N=751\end{array}$ & $\begin{array}{c}\mathrm{BSO} \geq 45 \\
\mathrm{~N}=532\end{array}$ & $\begin{array}{r}\mathrm{P} \\
\text { value }\end{array}$ \\
\hline Age (years) & $61.0(0.3)$ & $57.1(0.8)$ & $65.3(0.6)$ & 0.001 \\
\hline Race/Ethnicity (\%) & & & & 0.001 \\
\hline Non-Hispanic White & 78.8 & 83.4 & 82.2 & \\
\hline Non-Hispanic Black & 8.6 & 9.7 & 7.1 & \\
\hline Mexican American & 4.0 & 2.8 & 2.8 & \\
\hline Other & 8.6 & 4.1 & 7.9 & \\
\hline Some College degree or higher & 52.8 & 43.4 & 53.0 & 0.001 \\
\hline Living at or below poverty line (\%) & 17.5 & 18.7 & 14.7 & 0.277 \\
\hline Smoking status (\%) & & & & 0.001 \\
\hline former & 27.5 & 26.6 & 32.6 & \\
\hline Current & 17.2 & 27.2 & 11.3 & \\
\hline Age at menarche (years) & & & & 0.496 \\
\hline$<12$ & 18.9 & 21.4 & 19.3 & \\
\hline $12-14$ & 66.7 & 64.3 & 68.8 & \\
\hline$>14$ & 14.4 & 14.3 & 11.9 & \\
\hline Age at menopause & $45.7(0.2)$ & $35.6(0.3)$ & $49.5(0.2)$ & 0.001 \\
\hline \multicolumn{5}{|l|}{ Nulliparous } \\
\hline Ever use of Hormone therapy (\%) & 41.1 & 82.1 & 74.4 & 0.001 \\
\hline Sedentary lifestyle (\%) & 24.6 & 28.8 & 23.2 & 0.048 \\
\hline Waist Circumference (cm) & $95.5(0.3)$ & $96.8(0.6)$ & $94.4(0.7)$ & \\
\hline $\operatorname{BMI}\left(\mathrm{kg} / \mathrm{m}^{2}\right)$ & & & & 0.020 \\
\hline$<25.0$ & 35.9 & 29.5 & 39.3 & \\
\hline $25.0-29.9$ & 30.6 & 34.0 & 28.9 & \\
\hline$\geq 30.0$ & 33.5 & 36.4 & 31.9 & \\
\hline HDL Cholesterol (mg/dl) & $60.1(0.4)$ & $59.8(0.8)$ & $62.4(0.9)$ & 0.028 \\
\hline Total Cholesterol (mg/dl) & $215.0(0.9)$ & $217.6(2.0)$ & $217.3(2.1)$ & 0.349 \\
\hline C-reactive Protein (mg/dl) & $0.23(0.1-0.5)$ & $0.31(0.2-0.7)$ & $0.27(0.1-0.6)$ & 0.001 \\
\hline Hypertension (\%) & 44.7 & 47.7 & 58.2 & 0.001 \\
\hline Family history of diabetes (\%) & 35.5 & 42.1 & 35.6 & 0.025 \\
\hline Family history of Heart Attack (\%) & 15.4 & 22.0 & 18.1 & 0.002 \\
\hline Coronary Heart disease (\%) & 6.5 & 9.3 & 10.4 & 0.004 \\
\hline Cardiovascular disease (\%) & 9.5 & 12.4 & 13.8 & 0.006 \\
\hline
\end{tabular}

Values are survey weighted mean (Standard error), median (interquartile range) or percentages for continuous or categorical variables, respectively. BSO: Bilateral salpingo oophorectomy 


\section{The Study of Osteoporotic Fractures}

Descriptive statistics of both the Caucasian and African American cohorts are presented in table 4.5 according to diabetes and oophorectomy status. Participants in this cohort were relatively older than those in the NHANES study with the mean age of 71.8 years. Approximately $61 \%$ of women in this study had a high school education or less, with $20.3 \%$ being obese. Additionally, $39.6 \%$ of study participants were hypertensive while $10.3 \%$ reported being current smokers. African Americans made up 5.2\% of the entire cohort. The prevalence of hysterectomy, bilateral oophorectomy and diabetes was $28.2 \%, 21.7 \%$ and $7.2 \%$ respectively.

Uterine fibroid was the most frequently reported indication for surgery in women with either hysterectomy (38.8\%) or bilateral oophorectomy (45\%). A greater proportion of women with diabetes had undergone an oophorectomy than non-diabetic women (25.1 vs. 21.4). Furthermore, diabetics were more likely to be obese (36.4\% vs.19\%), African American (11.7\% vs. 4.7\%), have no college education (71.8\% vs. 60.9\%), hypertensive (54.8\% vs. $38.4 \%)$ and had undergone a hysterectomy (32.6\% vs. $27.9 \%)$, but were also less likely to be either former (27.4\% vs. $33.2 \%)$ or current (6.3\% vs. $12 \%)$ hormone therapy users compared to non-diabetics.

Among diabetic women, statistically significant differences between those with intact ovaries and bilateral oophorectomy were found with regards to age at menopause, parity, hormone therapy use and the prevalence of breast cancer. 
Table 4.5 Baseline characteristic of 7,950 postmenopausal women according to diabetes and bilateral oophorectomy status, The Study of Osteoporotic Fractures

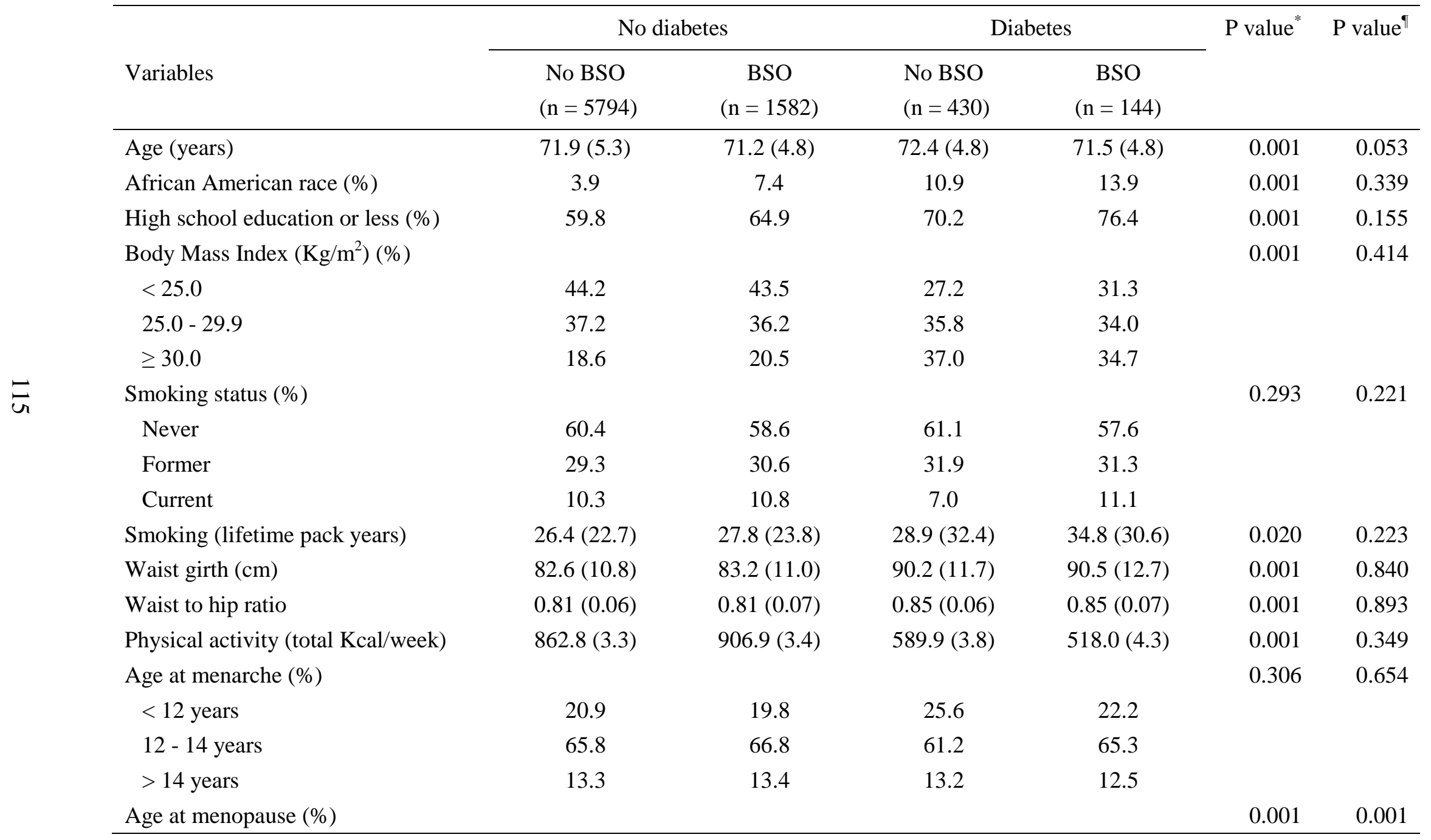




\begin{tabular}{|c|c|c|c|c|c|c|}
\hline$<45$ years & 16.8 & 39.0 & 17.2 & 46.5 & & \\
\hline $45-49$ years & 29.9 & 28.7 & 27.9 & 27.8 & & \\
\hline$>49$ years & 53.3 & 32.3 & 54.9 & 25.7 & & \\
\hline Parity & & & & & 0.002 & 0.029 \\
\hline None & 19.2 & 22.0 & 18.6 & 21.5 & & \\
\hline $1-2$ live births & 41.1 & 43.2 & 38.1 & 45.8 & & \\
\hline 3 - 4 live births & 30.0 & 27.6 & 30.7 & 25.7 & & \\
\hline$\geq 5$ live births & 9.7 & 7.2 & 12.6 & 6.9 & & \\
\hline Hormone therapy use & & & & & 0.001 & 0.001 \\
\hline Never & 60.8 & 33.2 & 74.9 & 40.6 & & \\
\hline Former & 30.8 & 41.6 & 21.6 & 44.8 & & \\
\hline Current & 8.4 & 25.2 & 3.5 & 14.6 & & \\
\hline \multicolumn{7}{|l|}{ Personal history of (\%) } \\
\hline Hypertension & 37.4 & 42.1 & 55.5 & 52.8 & 0.001 & 0.573 \\
\hline Angina & 8.5 & 11.3 & 16.5 & 13.2 & 0.001 & 0.343 \\
\hline Myocardial infarction & 5.4 & 6.1 & 10.9 & 11.8 & 0.001 & 0.773 \\
\hline Stroke & 2.4 & 3.8 & 6.5 & 8.1 & 0.001 & 0.557 \\
\hline Breast Cancer & 4.6 & 6.1 & 7.7 & 14.6 & 0.001 & 0.014 \\
\hline \multicolumn{7}{|l|}{ Serology measures of } \\
\hline HDL cholesterol (mg/dl) & $53.5(14.8)$ & $56.2(16.2)$ & $46.6(13.1)$ & $49.3(12.6)$ & 0.002 & 0.515 \\
\hline LDL cholesterol (mg/dl) & $154.6(34.4)$ & $146.4(37.1)$ & $155.0(54.0)$ & $135.2(32.6)$ & 0.071 & 0.252 \\
\hline Triglycerides (mg/dl) & $156.9(80.6)$ & $174.5(93.9)$ & $249.4(147)$ & $252.3(124)$ & 0.001 & 0.949 \\
\hline
\end{tabular}

Values are displayed in percent or mean (Standard deviation)

*P value comparing all four categories

"P value for subgroup analyses restricted to only women with diabetes

$P$ values based on Chi-square test for categorical variables and ANOVA for normally distributed continuous variables 


\section{Sex-steroid hormones levels according to diabetes and oophorectomy status}

Among the 894 women randomly selected from the Caucasian cohort in whom measures of sex hormones were available, statistically significant differences were found with regards to diabetes or ovarian status. Women with diabetes had lower SHBG ( $p=-$ $.001)$ and higher free testosterone $(\mathrm{p}=0.006)$, and percent free testosterone $(\mathrm{p}=0.001)$ than non-diabetics. On the other hand, women with diabetes also had lower levels of DHEAS. Furthermore, diabetic women had higher levels of estrone than non-diabetics. Conversely levels of SHBG were lower in diabetic women compared to those without a history of physician diagnosed diabetes $(\mathrm{p}=0.001)$.

Ovarian status influenced significantly the distribution of sex steroid hormones in this postmenopausal cohort. As expected, total testosterone $(\mathrm{p}=0.001)$ and free testosterone $(\mathrm{p}=0.001)$ were lower in women with bilateral oophorectomy than in women with intact ovaries, while no differences were found in estradiol $(\mathrm{p}=0.218)$ or DHEAS levels $(\mathrm{p}=0.266)$.

Ovarian status among diabetic women did profoundly influence sex hormone levels (table 4.6). For instance, diabetic women with oophorectomy were observed to have significantly elevated levels of both total testosterone and estradiol than diabetics with intact ovaries, even after adjustment for age and BMI.

Finally no association between total testosterone $(\mathrm{p}=0.103)$, free testosterone $(\mathrm{p}=0.456)$, total estradiol $(\mathrm{p}=0.4578)$, dehydroepiandrosterone sulfate $(\mathrm{p}=0.865)$, bioavailable estradiol $(\mathrm{p}=0.235)$ or free estradiol $(\mathrm{p}=0.212)$ with incident cardiovascular disease mortality was identified (table 4.7). 
Table 4.6 Hormone levels [median (IQR)] according to diabetes and bilateral oophorectomy status, the Study of Osteoporotic Fractures

\begin{tabular}{|c|c|c|c|c|c|c|c|}
\hline \multirow[b]{2}{*}{ Variables } & \multicolumn{4}{|c|}{ Oophorectomy and diabetes status } & \multirow[b]{2}{*}{ P value* } & \multirow[b]{2}{*}{$P$ value ${ }^{\pi}$} & \multirow[b]{2}{*}{ P value ${ }^{\S}$} \\
\hline & $\begin{array}{l}\text { No Diabetes or } \\
\text { Oophorectomy } \\
\qquad(\mathrm{n}=646)\end{array}$ & $\begin{array}{c}\text { Physician } \\
\text { diagnosed Diabetes } \\
(\mathrm{n}=69)\end{array}$ & $\begin{array}{l}\text { Bilateral } \\
\text { Oophorectomy } \\
(\mathrm{n}=162)\end{array}$ & $\begin{array}{c}\text { Oophorectomy\& } \\
\text { Diabetes } \\
(\mathrm{n}=17)\end{array}$ & & & \\
\hline Total Testosterone (ng/dl) & $20.0(12.0-30.0)$ & $21.0(11.0-33.0)$ & $12.0(7.5-17.0)$ & $12.0(6.0-16.0)$ & 0.001 & 0.012 & 0.016 \\
\hline Free Testosterone $(\mathrm{pg} / \mathrm{ml})$ & $2.2(1.2-3.5)$ & $2.7(1.6-4.1)$ & $1.2(0.7-1.8)$ & $1.8(1.3-2.9)$ & 0.001 & 0.071 & 0.301 \\
\hline$\%$ Free testosterone & $1.1(0.9-1.4)$ & $1.4(1.1-1.7)$ & $1.1(0.8-1.3)$ & $1.8(1.5-1.9)$ & 0.001 & 0.152 & 0.142 \\
\hline DHEAS (ug/dl) & $47.0(26.0-74.0)$ & $45.0(21.0-71.0)$ & $39.5(23.0-72.0)$ & $46.0(29.0-61.0)$ & 0.692 & 0.987 & 0.834 \\
\hline Estradiol (pg/ml) & $7.0(5.0-9.0)$ & $7.0(6.0-10.0)$ & $6.0(4.0-10.0)$ & $12.5(6.5-23.0)$ & 0.003 & 0.004 & 0.015 \\
\hline Estrone (pg/ml) & $22.0(15.5-29.0)$ & $28.0(22.0-32.0)$ & $23.5(14.0-36.0)$ & $31.0(14.0-55.0)$ & 0.036 & 0.460 & 0.122 \\
\hline SHBG (ug/dl) & $1.3(1.0-1.9)$ & $0.9(.06-1.2)$ & $1.6(1.1-2.3)$ & $0.8(0.3-2.0)$ & 0.001 & 0.854 & 0.502 \\
\hline
\end{tabular}

${ }^{*}$ P value comparing all four categories based on ANOVA for normally distributed continuous variables after log transformation (total testosterone, free testosterone, \% free testosterone, estradiol, estrone and DHEAS) or Kruskal-Wallis test for non-normally distributed continuous variables (SHBG).

${ }^{\pi} \mathrm{P}$ value for subgroup analyses restricted to only women with diabetes based on t-test for normally distributed variables (Free testosterone, $\%$ Free testosterone, estradiol and estrone) and Mann-Whitney U test for non-normally distributed variables (total testosterone, DHEAS and SHBG).

${ }^{\S} \mathrm{P}$ value for subgroup analyses restricted to only women with diabetes adjusted for BMI, based on ANCOVA for normally distributed data and rank analyses of covariance for non-normally distributed data. 
Table 4.7: Association of free testosterone, total testosterone and dehydroepiandrosterone sulfate and risk of CVD mortality, the Study of Osteoporotic Fractures

\begin{tabular}{|c|c|c|c|c|c|c|c|c|}
\hline & \multicolumn{2}{|c|}{ unadjusted } & \multicolumn{2}{|c|}{ Model 1} & \multicolumn{2}{|c|}{ Model 2} & \multicolumn{2}{|c|}{ Model 3} \\
\hline & $\mathrm{HR}(95 \% \mathrm{CI})$ & $\mathrm{P}$ value & HR (95\%CI) & $\mathrm{P}$ value & $\mathrm{HR}(95 \% \mathrm{CI})$ & $\mathrm{P}$ value & HR (95\%CI) & $P$ value \\
\hline \multicolumn{9}{|c|}{ Free Testosterone } \\
\hline $\mathrm{Q} 1(<1.1 \mathrm{pg} / \mathrm{ml})$ & 1.0 (Referent) & & 1.0 (Referent) & & 1.0 (Referent) & & 1.0 (Referent) & \\
\hline Q2 (1.1 - $1.7 \mathrm{pg} / \mathrm{ml})$ & $1.06(0.66-1.70)$ & 0.798 & $1.20(0.75-1.92)$ & 0.452 & $1.00(0.57-1.74)$ & 0.996 & $1.03(0.59-1.82)$ & 0.912 \\
\hline Q3 $(1.8-2.9 \mathrm{pg} / \mathrm{ml})$ & $0.91(0.57-1.46)$ & 0.708 & $0.99(0.62-1.59)$ & 0.964 & $0.97(0.60-1.69)$ & 0.924 & $0.73(0.41-1.33)$ & 0.668 \\
\hline $\mathrm{Q} 4(>2.9 \mathrm{pg} / \mathrm{ml})$ & $0.87(0.55-1.40)$ & 0.567 & $0.92(0.57-1.47)$ & 0.729 & $0.90(0.52-1.58)$ & 0.724 & $0.88(0.50-1.57)$ & 0.306 \\
\hline Risk per 1 SD increase & $0.97(0.82-1.16)$ & 0.770 & $0.96(0.81-1.14)$ & 0.632 & $0.99(0.80-1.22)$ & 0.914 & $0.92(0.73-1.15)$ & 0.456 \\
\hline \multicolumn{9}{|c|}{ Total Testosterone } \\
\hline $\mathrm{Q} 1(<11.0 \mathrm{ng} / \mathrm{dl})$ & 1.0 (Referent) & & 1.0 (Referent) & & 1.0 (Referent) & & 1.0 (Referent) & \\
\hline Q2 (11.0 - $16.9 \mathrm{ng} / \mathrm{dl})$ & $0.94(0.59-1.50)$ & 0.799 & $1.00(0.63-1.60)$ & 0.990 & $0.81(0.47-1.40)$ & 0.457 & $0.67(0.37-1.18)$ & 0.162 \\
\hline Q3 (17.0 - $26.9 \mathrm{ng} / \mathrm{dl})$ & $0.99(0.63-1.56)$ & 0.963 & $1.12(0.71-1.77)$ & 0.633 & $1.25(0.74-2.11)$ & 0.398 & $1.30(0.76-2.23)$ & 0.345 \\
\hline Q4 (>26.9 ng/dl) & $0.71(0.43-1.18)$ & 0.186 & $0.71(0.43-1.17)$ & 0.183 & $0.60(0.33-1.09)$ & 0.096 & $0.60(0.32-1.10)$ & 0.099 \\
\hline Risk per 1 SD increase & $0.86(0.71-1.06)$ & 0.149 & $0.85(0.71-1.03)$ & 0.107 & $0.82(0.66-1.03)$ & 0.079 & $0.83(0.66-1.04)$ & 0.103 \\
\hline \multicolumn{9}{|c|}{ DHEA sulfate (ug/dl) } \\
\hline $\mathrm{Q} 1(<25.0 \mathrm{ug} / \mathrm{dl})$ & 1.0 (Referent) & & 1.0 (Referent) & & 1.0 (Referent) & & 1.0 (Referent) & \\
\hline Q2 (25.0 - $42.9 \mathrm{ug} / \mathrm{dl})$ & $1.05(0.68-1.63)$ & 0.832 & $1.09(0.70-1.69)$ & 0.707 & $0.90(0.53-1.52)$ & 0.693 & $0.94(0.55-1.62)$ & 0.832 \\
\hline Q3 (43.0 - $71.9 \mathrm{ug} / \mathrm{dl})$ & $0.53(0.31-0.89)$ & 0.016 & $0.57(0.34-0.97)$ & 0.036 & $0.62(0.36-1.09)$ & 0.099 & $0.71(0.40-1.27)$ & 0.250 \\
\hline $\mathrm{Q} 4(>71.9 \mathrm{ug} / \mathrm{dl})$ & $0.80(0.50-1.26)$ & 0.343 & $0.98(0.61-1.56)$ & 0.921 & $0.97(0.57-1.64)$ & 0.905 & $0.90(0.52-1.58)$ & 0.719 \\
\hline Risk per 1 SD increase & $0.90(0.74-1.09)$ & 0.257 & $0.99(0.81-1.19)$ & 0.889 & $1.03(0.84-1.24)$ & 0.787 & $1.02(0.84-1.24)$ & 0.865 \\
\hline
\end{tabular}

MODEL 1; adjusted for age, education

MODEL 2; adjusted for Model 1 plus age at menopause bilateral oophorectomy status, hysterectomy status, parity and estrogen use MODEL 3, adjusted for Model 2 BMI, waist hip ratio, hypertension, prevalent CVD, diabetes smoking status, and physical activity 


\section{Prevalent cardiovascular disease in NHANES}

Among participants in NHANES III, a greater proportion of prevalent cardiovascular disease (20\%) was found in oophorectomized women with diabetes.

Diabetes increased the risk of both coronary heart disease and cardiovascular disease as expected. On the other hand, oophorectomy overall did not increase the CHD or CVD risk statistically (table 4.8). The increased in cardiovascular disease risk in oophorectomized women with diabetes was influenced by timing of oophorectomy, however. Women who had this surgical procedure before age 45 years had a twofold increased odds for both CHD (OR: 2.65, 95\%CI: 1.08 - 6.52) and CVD (OR: 2.40, 95\% CI: 1.02 - 5.66) compared to women with no oophorectomy or diabetes.

In analyses restricted to women with diabetes, the risk of CVD with early oophorectomy (<45 years) remained elevated in age-adjusted models (OR: $2.40,95 \% \mathrm{CI}$ : 1.33-4.33). However, in multivariable adjusted models, the estimates became attenuated with no statistically significant risk identified in diabetic women with oophorectomy before age 45 years or diabetic women who had oophorectomy after age 45 years when compared to women with diabetes only.

Similarly, in NHANES 1999-2010, the joint effect of diabetes and oophorectomy on cardiovascular disease did not approach statistical significance, but once again, diabetic women who had oophorectomy before age 45 years had elevated odds for coronary heart disease (OR: 1.85, 95\%CI: 1.15-2.97) and cardiovascular disease (OR: 2.11, 95\%CI: 1.45-3.08) (table 4.9) when compared to women with no oophorectomy or diabetes. 
Table 4.8 Odds of prevalent cardiovascular disease according to diabetes and oophorectomy status, Third National Health and Nutrition Examination Survey (1988-1994)

\begin{tabular}{lccc}
\hline & & $\begin{array}{c}\text { Coronary Heart } \\
\text { disease }\end{array}$ & $\begin{array}{c}\text { Cardiovascular } \\
\text { disease }^{2}\end{array}$ \\
\cline { 2 - 4 } & $\mathrm{N}$ & OR (95\%CI) & OR (95\%CI) \\
\hline No oophorectomy/diabetes & 1695 & 1 (Referent) & 1 (Referent) \\
Diabetes & 812 & $1.88(1.06-3.33)$ & $2.14(1.42-3.21)$ \\
Oophorectomy & 506 & $1.32(0.75-2.30)$ & $1.20(0.71-2.03)$ \\
Diabetes and oophorectomy & 211 & $1.83(0.81-4.16)$ & $1.72(0.80-3.70)$ \\
Oophorectomy before age 45 & 124 & $2.65(1.08-6.52)$ & $2.40(1.02-5.66)$ \\
Oophorectomy at or after age 45 & 87 & $0.78(0.19-3.29)$ & $0.95(0.34-2.67)$ \\
\hline
\end{tabular}

OR: Odds ratio CI: Confidence interval

${ }^{1}$ Model adjusted for age, race, education, age at menopause, hormone therapy use, hysterectomy status, Body Mass Index, Hypertension, smoking status, family history of Heart disease, physical activity and total Cholesterol.

${ }^{2}$ Adjusted for 1 and age at menarche, HDL cholesterol and white blood cell count, 
Table 4.9 Odds of prevalent cardiovascular disease according to diabetes and oophorectomy status, National Health and Nutrition Examination Survey (1999-2010)

\begin{tabular}{lccc}
\hline & & $\begin{array}{c}\text { Coronary Heart } \\
\text { disease }\end{array}$ & $\begin{array}{c}\text { Cardiovascular } \\
\text { disease }\end{array}$ \\
\cline { 2 - 4 } & $\mathrm{N}$ & OR $(95 \% \mathrm{CI})$ & OR (95\%CI) \\
\hline No oophorectomy diabetes & 3689 & 1 (Referent) & 1 (Referent) \\
Diabetes & 1034 & $1.93(1.47-2.52)$ & $1.86(1.46-2.37)$ \\
Oophorectomy & 1283 & $1.14(0.78-1.66)$ & $1.06(0.78-1.44)$ \\
Diabetes and oophorectomy & 369 & $1.42(0.95-2.12)$ & $1.63(1.16-2.30)$ \\
Oophorectomy before age 45 & 207 & $1.85(1.15-2.97)$ & $2.11(1.45-3.08)$ \\
Oophorectomy at or after age 45 & 162 & $0.77(0.40-1.47)$ & $0.98(0.54-1.78)$
\end{tabular}

OR: Odds ratio CI: Confidence interval

Model adjusted for age, race, , education, age at menarche, age at menopause, Hormone therapy use, hysterectomy status, Body Mass Index, Hypertension, smoking status, family history of Heart disease, physical inactivity, total Cholesterol, HDL cholesterol and C-reactive protein, 
In analyses restricted to diabetic women in NHANES 1999-2010, s significant interaction was found between diabetes status and age at oophorectomy $(\mathrm{p}=0.001)$. Oophorectomy after age 45 on the other hand was associated with a reduction in cardiovascular disease (OR: 0.49, 95\%CI: 0.25-0.95) (table 4.10). Moreover, comparing diabetic women who had oophorectomy before age 45 years to diabetic women who had this surgical procedure after age 45 years, the odds of CVD was threefold higher among the former (OR: 3.05, 95\%CI: 1.64-5.67).

Table 4.10 Odds of prevalent cardiovascular disease among diabetics by age at oophorectomy, National Health and Nutrition Examination Survey (1999-2010)

\begin{tabular}{lccc}
\hline & & $\begin{array}{c}\text { Coronary Heart } \\
\text { disease }\end{array}$ & $\begin{array}{c}\text { Cardiovascular } \\
\text { disease }\end{array}$ \\
\cline { 2 - 4 } & $\mathrm{N}$ & OR (95\%CI) & OR (95\%CI) \\
\hline $\begin{array}{l}\text { Diabetes without oophorectomy } \\
\text { Diabetes and oophorectomy }\end{array}$ & 1034 & 1 (Referent) & 1 (Referent) \\
before age 45 & 207 & $1.10(0.66-1.84)$ & $1.50(0.94-2.39)$ \\
Diabetes and oophorectomy & & & \\
at or after age 45 & 162 & $0.33(0.16-0.70)$ & $0.49(0.25-0.95)$ \\
Diabetes \& oophorectomy $<45$ vs. & & $3.35(1.57-7.14)$ & $3.05(1.64-5.67)$ \\
Diabetes \& oophorectomy $\geq 45$ & & & \\
\hline
\end{tabular}

OR: Odds ratio CI: Confidence interval

Model adjusted for age, race, , education, age at menarche, age at menopause, Hormone therapy use, hysterectomy status, Body Mass Index, Hypertension, smoking status, family history of Heart disease, physical inactivity, HDL cholesterol and hemoglobin A1c,. 


\section{Incident cardiovascular disease in SOF study}

During follow-up, 2,652 (38\%) non fatal cases of angina, myocardial infarction, stroke, congestive heart failure and other forms of heart diseases were documented in 6,976 women who were free of disease.

In analyses based on a discrete time logit model adjusted for age, the risk of incident cardiovascular disease was elevated for women with diabetes (OR: 1.33 95\%CI:1.16-1.51) as well as women with oophorectomy (OR:1.16, 95\% CI:1.08-1.24) in age adjusted models (table 4.11). After further adjustment for sociodemographic, anthropometric measures, reproductive and health behavioral/lifestyle factors, only diabetes was associated with incident CVD. A 9\% reduced risk of CVD was observed among women with both diabetes and oophorectomy, albeit this did not approach statistical significant.

In analyses restricted to diabetic women, ovarian status appeared to influence disease risk in fully adjusted models but again this association was not statistically significance (OR: $0.78,95 \%$ CI: 0.56-1.09). No interaction was present with respect to either general obesity $(\mathrm{P}=0.099)$, nor central obesity as measured by waist circumference $(\mathrm{p}=0.542)$ or waist to hip ratio $(\mathrm{p}=0.538)$. 
Table 4.11: The risk of incident non-fatal cardiovascular disease according to diabetes and bilateral oophorectomy status, the Study of Osteoporotic Fractures

\begin{tabular}{lccc}
\hline & & Age Adjusted & Multivariable adjusted $^{\text {II }}$ \\
\cline { 2 - 4 } Variables & $\mathrm{N}$ & OR (95\%CI) & OR (95\%CI) \\
\hline All Participants & & & \\
No Diabetes \& No Oophorectomy & 5155 & 1 (Referent) & 1 (Referent) \\
Diabetes without oophorectomy & 343 & $1.33(1.16-1.51)$ & $1.20(1.04-1.37)$ \\
Oophorectomy without diabetes & 1364 & $1.16(1.08-1.24)$ & $1.04(0.92-1.18)$ \\
Diabetes \& Oophorectomy & 114 & $1.11(0.88-1.41)$ & $0.91(0.70-1.20)$ \\
Diabetic women & & & 1 (Referent) \\
No Oophorectomy & 343 & $0.87(0.66-1.14)$ & $0.78(0.56-1.09)$ \\
Oophorectomy & 114 & & \\
\hline
\end{tabular}

${ }^{\mathbb{I}}$ Adjusted for baseline self-reported measures of age, race, education, age at menopause, hysterectomy status, parity, postmenopausal hormone use, BMI, waist hip ratio, hypertension, smoking status and physical activity 


\section{Age at natural or surgical menopause and risk of incident CVD in SOF study}

The multivariable adjusted spline regression model showed a decreasing trend

in the odds for incident cardiovascular disease as menopausal age increased, an

observation which was present in women with or without diabetes. An early age at

oophorectomy or natural menopause was associated with an increased CVD risk which

attenuated with each year that menopause was delayed (Figure 4.1).

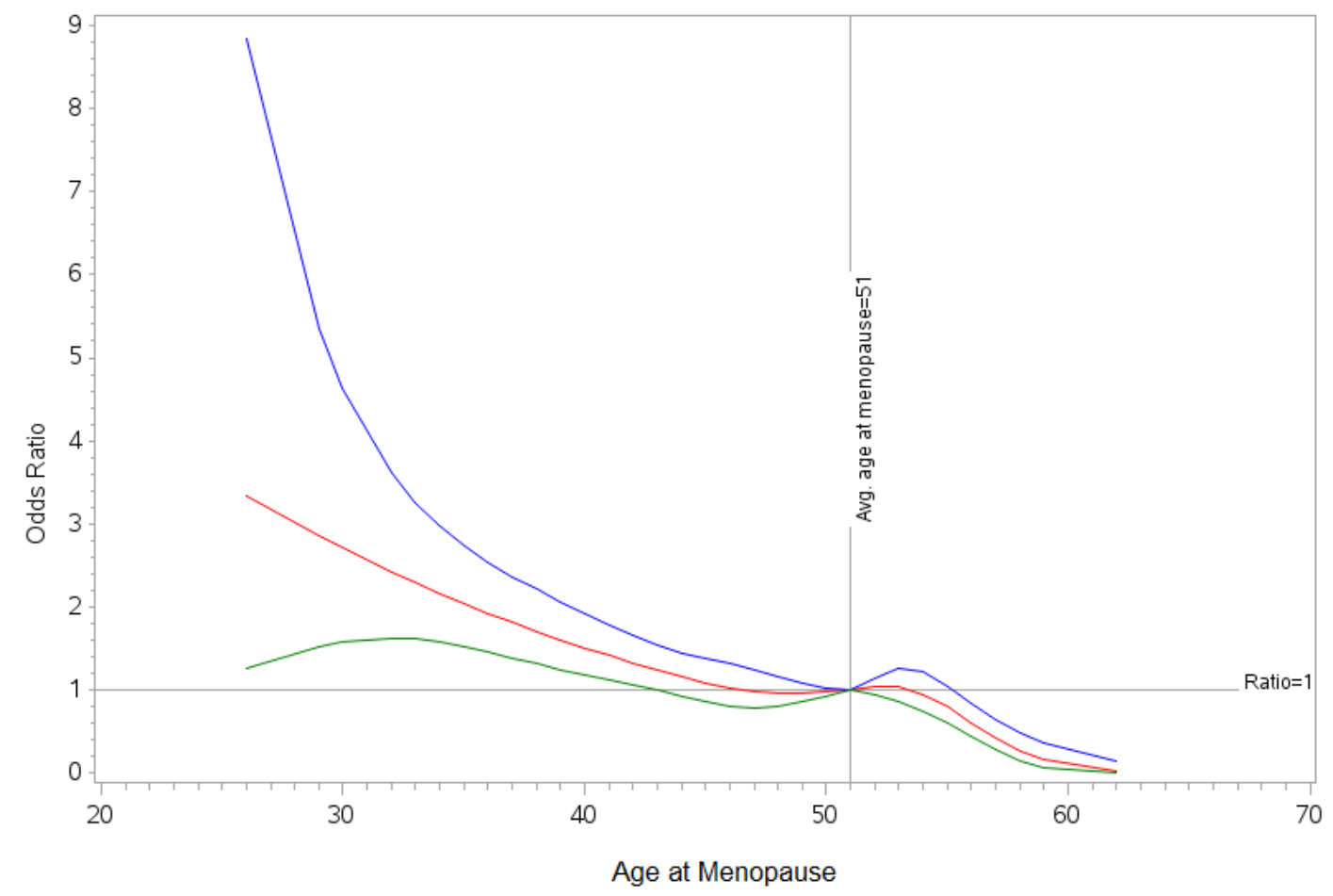

Figure 4.1 Odds ratio and 95\% CI estimates for age at menopause and the incidence of cardiovascular disease, the Study of Osteoporotic Fractures. The average age at natural menopause (51 years) was used as reference value with odds ratio of 1.00. Model was adjusted for diabetes and oophorectomy status, age, race, education, parity, postmenopausal hormone use, BMI, waist to hip ratio, hypertension, smoking status and physical activity. Red curve: Odds ratio, blue curve: upper limit of $95 \%$ confidence interval, green curve: lower limit of $95 \%$ confidence interval, 
With the reported average age at menopause (51 years) as reference value, women who reached menopause after this age appeared to have a statistically significant reduced risk of incident cardiovascular disease.

On the other hand, oophorectomy after age 51 did not increase nor decrease the risk for cardiovascular disease as the upper limit of the $95 \%$ confidence interval was always above the null value (Odds ratio $=1.00)$ (figure 4.2) .

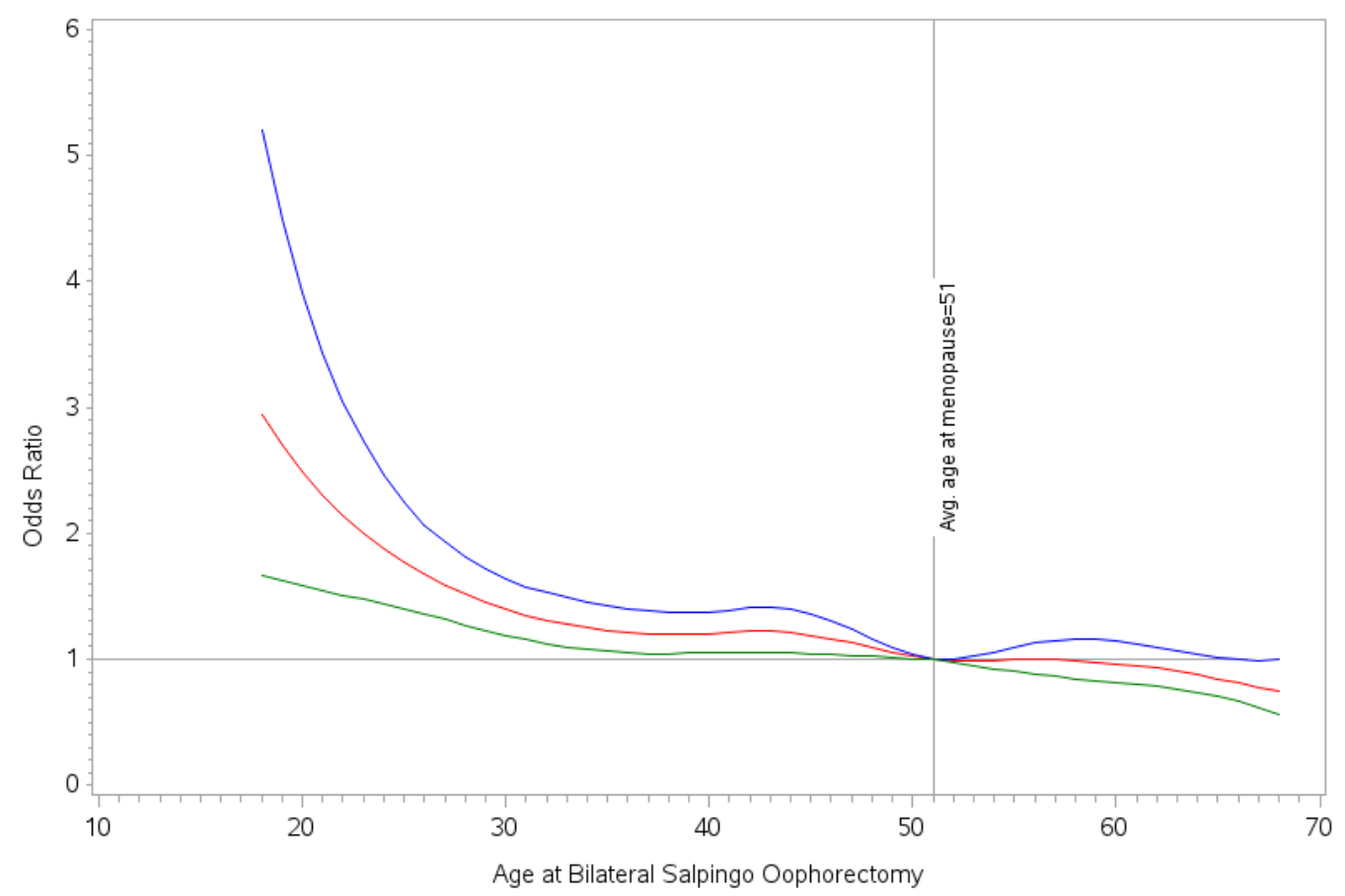

Figure 4.2 Odds ratio and 95\%CI estimates for age at bilateral oophorectomy and the incidence of cardiovascular disease, the Study of Osteoporotic Fractures. The average age at natural menopause (51 years) was used as reference value with odds ratio of 1.00. Model was adjusted for diabetes and oophorectomy status, age, race, education, parity, postmenopausal hormone use, BMI, waist to hip ratio, hypertension, smoking status and physical activity. Red curve: Odds ratio, blue curve: upper limit of $95 \%$ confidence interval, green curve: lower limit of $95 \%$ confidence interval, 


\section{Cardiovascular disease Mortality}

During a mean follow-up of 12.7 years, 1,395 deaths were documented with $46 \%$ of all reported deaths attributed to CVD in NHANES III. Cardiovascular related mortality rate was 17.7 per 1000 person years. CVD mortality was highest among women with both diabetes and oophorectomy (20.9 per 1000 person years) (table 4.12). Having diabetes or diabetes and oophorectomy predisposed women to increased cardiovascular mortality (Figure 4.3). In age adjusted models, the risk of CVD mortality for participants were as follows: diabetes and oophorectomy (HR: 2.49, 95\%CI: 1.50-4.16), diabetes without oophorectomy (HR: 1.74, 95\%CI: 1.30-2.34) and oophorectomy without diabetes (HR: 1.22, 95\%CI: 0.84-1.79). In multivariable models with adjustments made for race/ethnicity, education, body mass index, smoking status, total cholesterol, hypertension, waist-to-hip ratio, age at menopause, hysterectomy, hormone therapy use, family history of MI and physical activity, the risk of CVD death was still elevated in women with both diabetes and oophorectomy (HR: 2.23, 95\%CI: 1.25-3.99) as well as diabetes without oophorectomy (HR: 1.79, 95\%CI: 1.22-2.62), while that for women with oophorectomy without diabetes did not attain statistical significance (HR:1.14, 95\%CI:

0.65-2.00) (table 4:13). In analyses restricted to only diabetic women, oophorectomy did not influence CVD mortality (HR: 1.44, 95\%CI: 0.93-2.22). However compared to women with diabetes and intact ovaries, diabetic women with oophorectomy before age 45 had a significantly elevated risk (HR: 1.92, 95\%CI: 1.06-3.48).

The rate of mortality attributable to cardiovascular disease in the Study of Osteoporotic Fractures, mortality after a mean follow up of 14.8 years was 14.6 per 1000 
person years. CVD mortality rates in women with diabetes without oophorectomy (28.9 per 1000 person years) and diabetes with oophorectomy (30.8 per 1000 person years) were twice as high as the rate for the whole SOF population.

Table 4.12: Incidence of cardiovascular disease related mortality, NHANES III (1988-1994)

\begin{tabular}{lccccc}
\hline Variables & N & Deaths & \% Deaths & $\begin{array}{c}\text { Mean follow- } \\
\text { up (yrs) }\end{array}$ & $\begin{array}{c}\text { Rate per 1000 } \\
\text { person years }\end{array}$ \\
\hline No Diabetes \& No Oophorectomy & 1695 & 356 & 15.3 & 12.05 & 17.4 \\
Diabetes without oophorectomy & 812 & 182 & 19.9 & 11.62 & 19.3 \\
Oophorectomy without diabetes & 506 & 94 & 15.5 & 12.64 & 14.7 \\
Diabetes \& Oophorectomy & 211 & 52 & 22.0 & 11.76 & 20.9 \\
\hline
\end{tabular}

Table 4.13: The risk of cardiovascular disease related mortality according to diabetes and bilateral oophorectomy status, NHANES III (1988-1994)

\begin{tabular}{lcc}
\hline \multirow{2}{*}{ Variables } & Age Adjusted & Multivariable adjusted ${ }^{\mathbb{I I}}$ \\
\cline { 2 - 3 } No Diabetes \& No Oophorectomy & 1 (Referent) & 1 (Referent) \\
Diabetes without oophorectomy & $1.74(1.30-2.34)$ & $1.79(1.22-2.62)$ \\
Oophorectomy without diabetes & $1.22(0.84-1.79)$ & $1.14(0.65-2.00)$ \\
Diabetes \& Oophorectomy & $2.49(1.50-4.16)$ & $2.23(1.25-3.99)$ \\
\hline
\end{tabular}




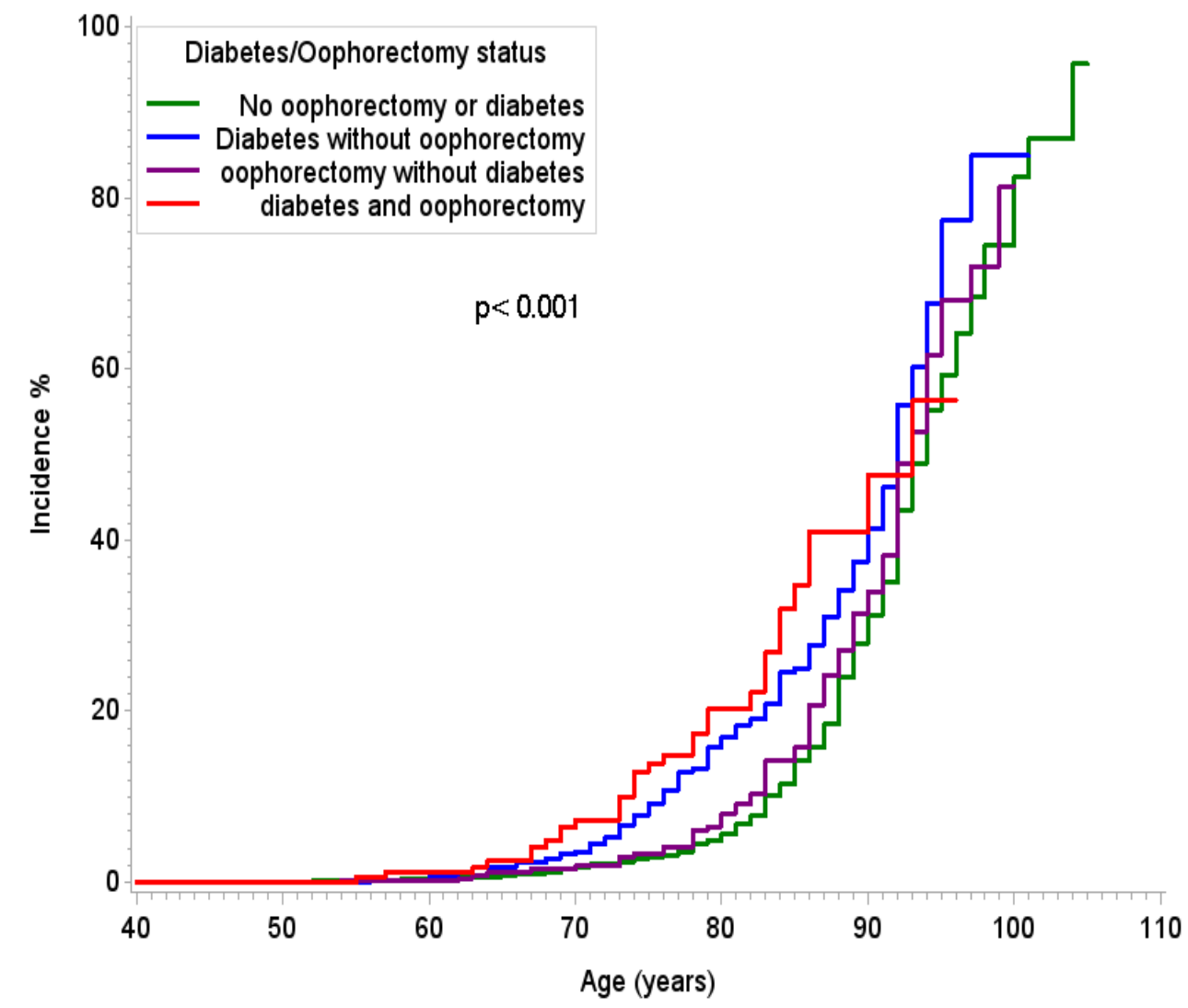

Figure 4.3 Kaplan-Meier cumulative incidence estimates of fatal CVD events among participants without a history of cardiovascular disease, NHANES (19881994)

Similar to the NHANES results, diabetic women with or without oophorectomy had elevated risk for CVD mortality (Figure 4.4). Oophorectomy appeared to have an adverse effect on women with diabetes. For instance, the risk of cardiovascular related mortality was two-and-half fold in women with diabetes and oophorectomy while women with diabetes but no oophorectomy had a twofold increased risk (table 4.14). Unlike NHANES III in which age at oophorectomy in diabetic women influenced CVD mortality risk, diabetic women in the SOF study who had oophorectomy before age 45 years had similar elevated risk as diabetic women who had oophorectomy at or after age 45 years when compared to women with neither oophorectomy nor diabetes. 
Additionally, in analyses restricted to diabetic women only, the risk of CVD mortality did not statistically differ by oophorectomy status (HR: 1.39, 95\%CI: $0.78-2.48$ ) or age at which oophorectomy was performed $(\mathrm{p}=0.457)$.

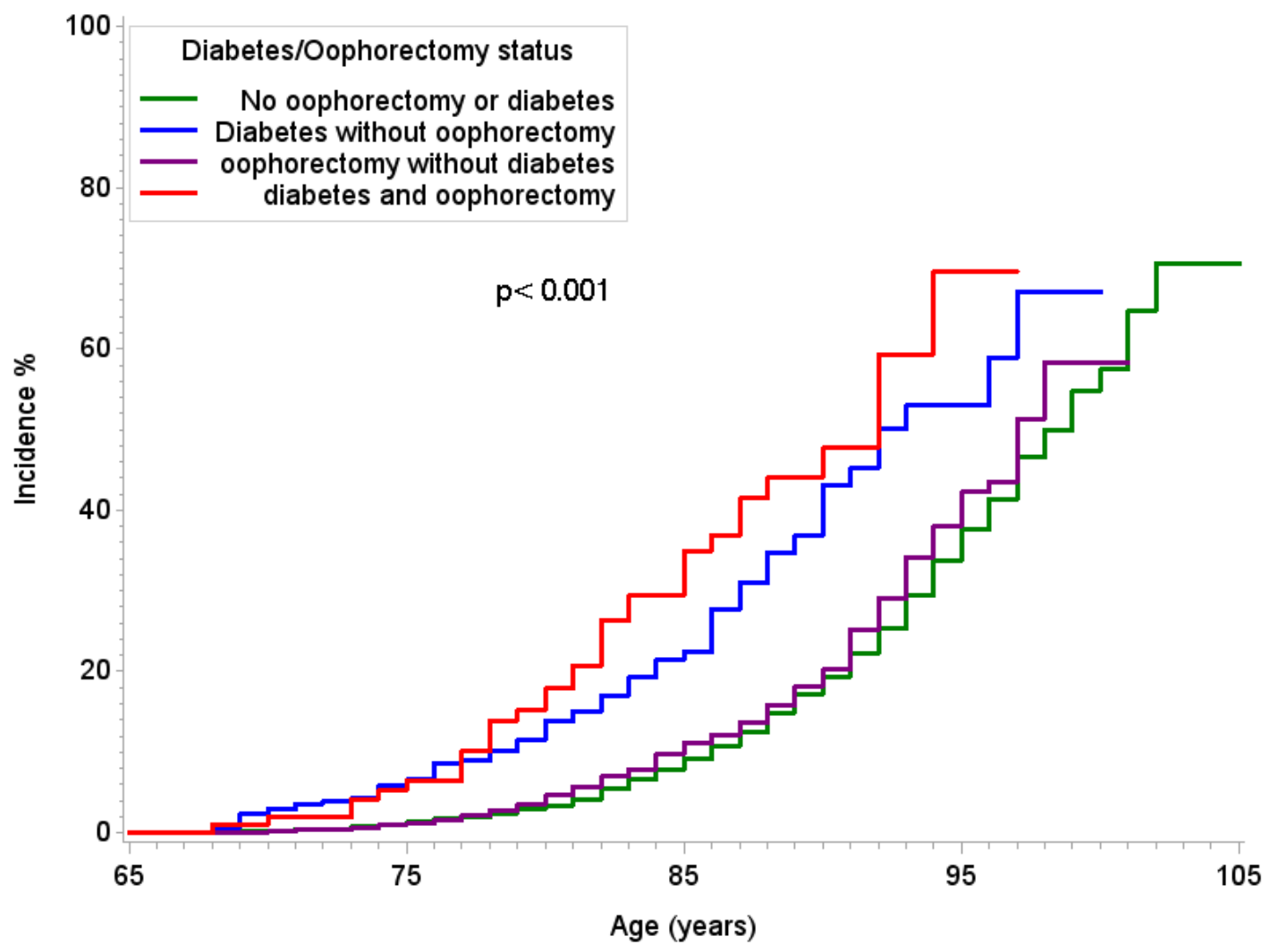

Figure 4.4 Kaplan-Meier cumulative incidence estimates of CVD death in participants without a history of heart disease, The Study of Osteoporotic Fractures. 
Table 4.14: The risk of all cause and cardiovascular disease related mortality according to diabetes and bilateral oophorectomy status, the Study of Osteoporotic Fractures

\begin{tabular}{lccc}
\hline & & Age Adjusted & Multivariable adjusted ${ }^{\mathbb{I}}$ \\
\cline { 2 - 4 } Variables & $\mathrm{N}$ & HR (95\%CI) & HR (95\%CI) \\
\hline All cause Mortality & & & \\
No Diabetes \& No Oophorectomy & 5568 & 1 (Referent) & 1 (Referent) \\
Diabetes without oophorectomy & 383 & $1.85(1.65-2.08)$ & $1.72(1.52-1.94)$ \\
Oophorectomy without diabetes & 1465 & $1.11(1.03-1.20)$ & $1.04(0.92-1.18)$ \\
Diabetes \& Oophorectomy & 124 & $2.32(1.91-2.82)$ & $2.17(1.73-2.72)$ \\
Cardiovascular Disease Mortality & & & \\
No Diabetes \& No Oophorectomy & 5568 & $1($ Referent $)$ & $1($ Referent) \\
Diabetes without oophorectomy & 383 & $2.40(2.01-2.88)$ & $1.99(1.65-2.39)$ \\
Oophorectomy without diabetes & 1465 & $1.15(1.01-1.31)$ & $1.10(0.90-1.35)$ \\
Diabetes \& Oophorectomy & 124 & $3.13(2.30-4.26)$ & $2.57(1.18-3.67)$ \\
\hline
\end{tabular}

${ }^{\mathbb{T}}$ Adjusted for baseline measures of age, education, age at menopause, hysterectomy status, parity, postmenopausal hormone use, BMI, waist hip ratio, hypertension, prior history of cardiovascular disease, diabetes, smoking status and physical activity

A summary of the estimates assessing the risk of cardiovascular disease in women with and without diabetes, based on results from the third National Health and Nutritional Examination Survey (1988-1994), the National Health and Nutritional Examination Survey 1999-2010 and the Study of Osteoporotic Fractures are illustrated by figure 4.5 and figure 4.6. Women who had oophorectomy before age 45 were found to have significantly increased risk for incident CVD compared to women without oophorectomy (OR: 1.17, 95\% CI: 1.04-1.37). Although early oophorectomy appeared to have an increased risk for CVD related mortality, this did not approach statistical significance (figure 4.7). 


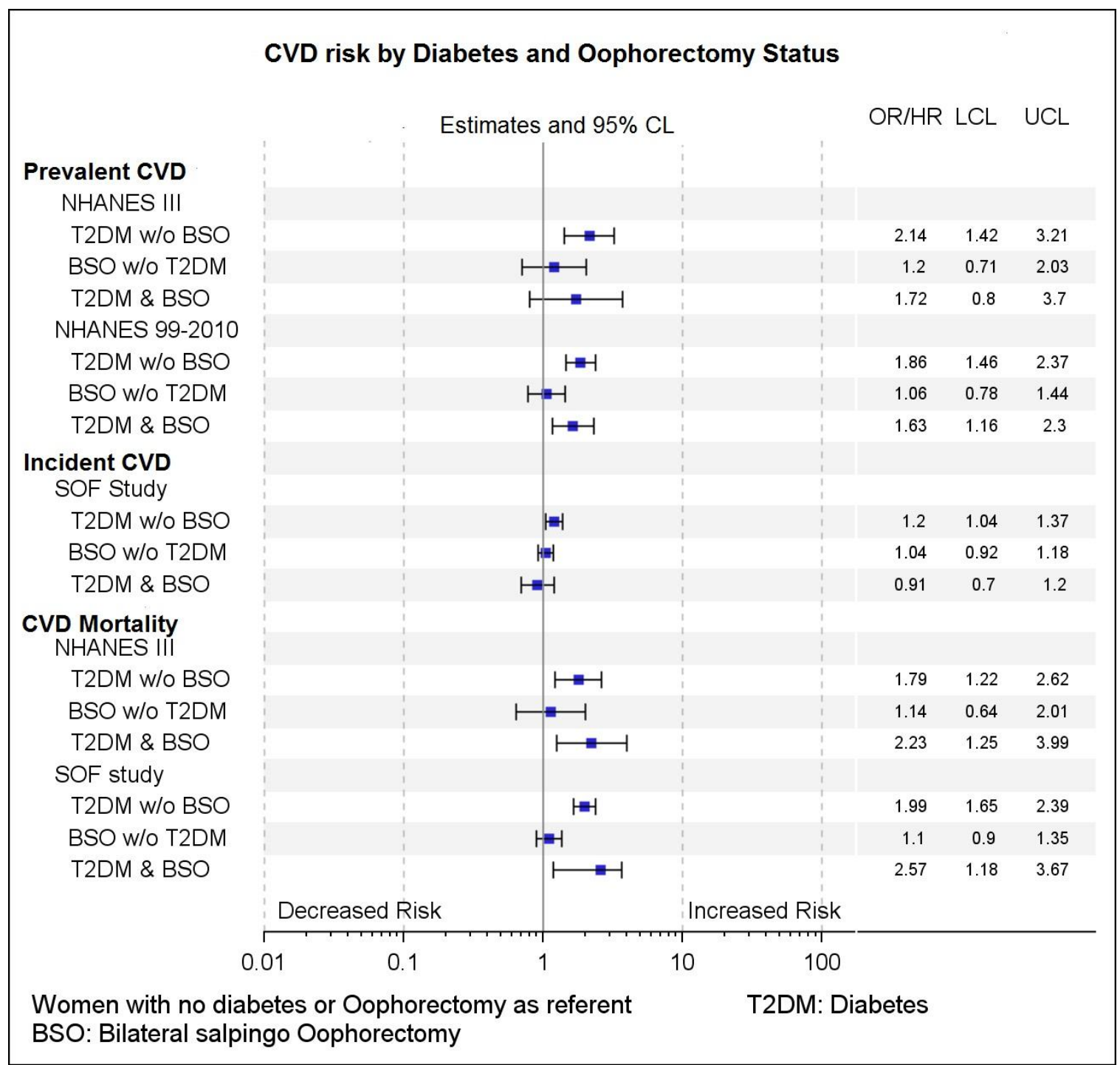

Figure 4.5 Summary estimates of the risk of cardiovascular disease according to diabetes and oophorectomy status 


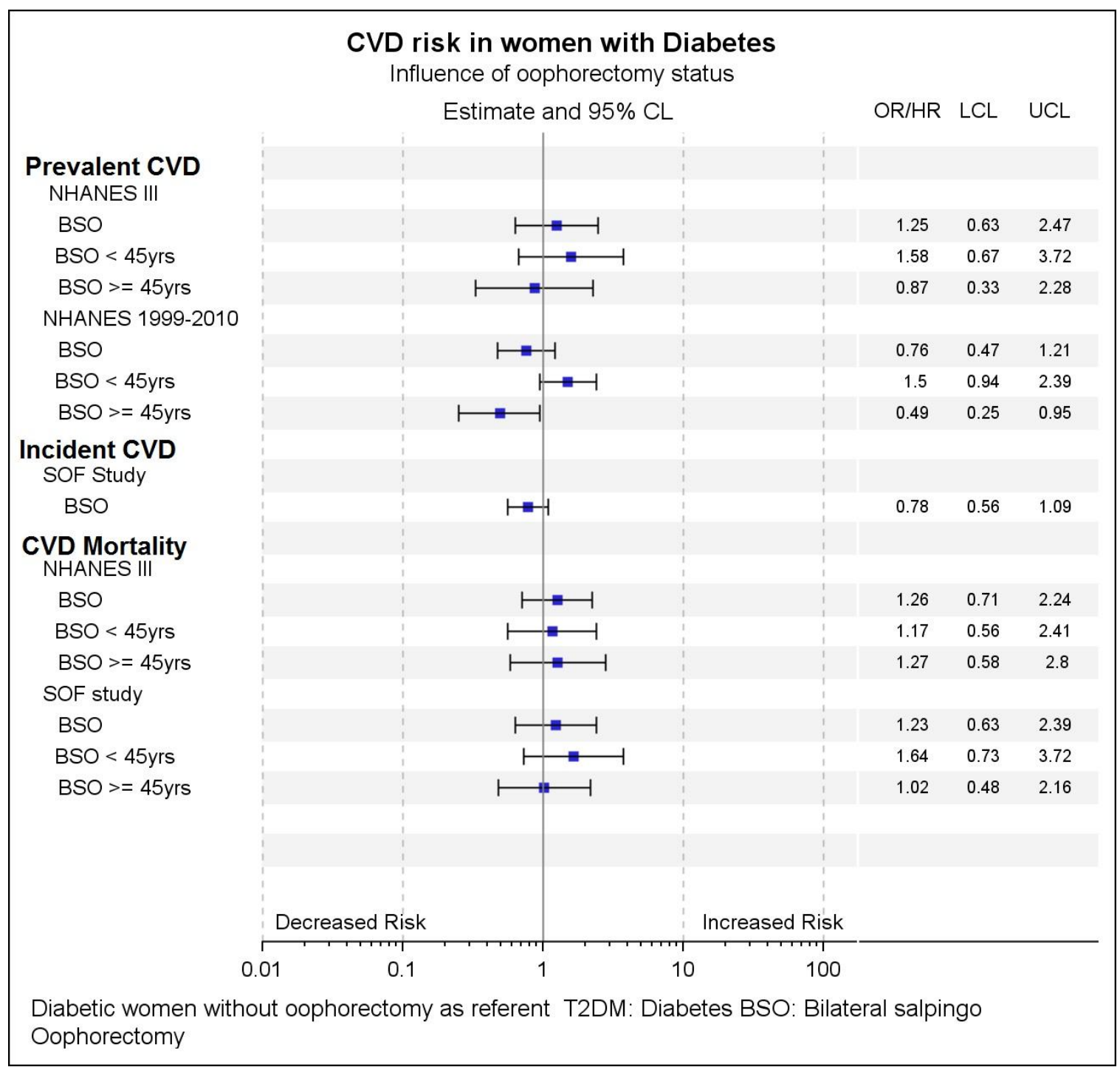

Figure 4.6 Summary estimates of the risk of cardiovascular disease in women with diabetes according to oophorectomy status as well as age at oophorectomy 


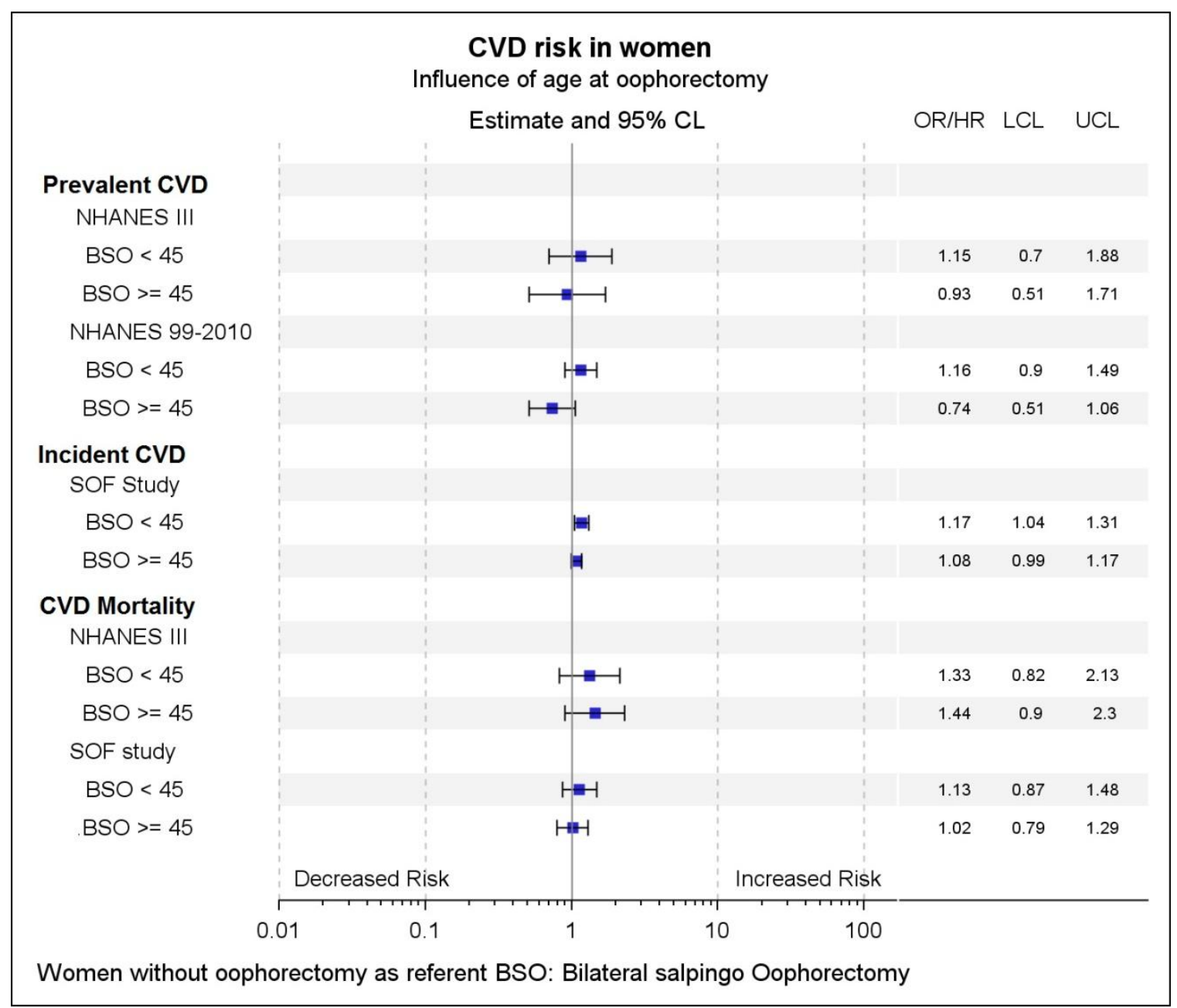

Figure 4.7 Summary estimates of the risk of cardiovascular disease in women according to age at oophorectomy 


\section{CHAPTER FIVE}

\section{DISCUSSION}

\section{Summary of findings}

This is the first population based study to assess the effect of bilateral salpingo oophorectomy on the risk of incident cardiovascular disease or cardiovascular disease related mortality among diabetic women. Because androgen levels are increased in diabetic women, we proposed that bilateral salpingo-oophorectomy would not be associated with the increased CVD risk reported in non-diabetic women, and might instead reduce the CVD in this at-risk population. Although diabetic women with oophorectomy were found to have reduced levels of androgens, this analysis of 17,549 postmenopausal women from three different studies did not support an overall protective effect of oophorectomy in diabetic women in reducing cardiovascular disease risk. This observation was consistent when diabetic women with bilateral oophorectomy were compared to either women with natural menopause without diabetes, or naturally menopausal diabetic women.

Contrary to our a priori hypothesis, oophorectomy increased the risk of cardiovascular disease mortality in diabetic women. 
Interestingly, the increased risk of adverse cardiovascular events in oophorectomized diabetic women appeared to be limited to women who had oophorectomy before the natural age average at menopause (51 years). Additionally, early age at natural menopause, either natural or through surgical removal of the ovaries, predisposed women to increased risk for non-fatal cardiovascular disease.

\section{Discussions based on study hypotheses}

\section{Hypothesis One}

When women end their reproductive years, there is an abrupt and profound decline in estradiol production by the ovary, and the only remaining source of estrogens is from androstenedione from the adrenals. By contrast, testosterone levels are relatively unchanged through the menopausal transition (293), but are about 50\% lower if the ovaries are removed surgically

In the present study, older postmenopausal women in the Study of Osteoporotic Fractures with bilateral oophorectomy were again found to have reduced levels of both free and total testosterone. Earlier studies of androgen levels in postmenopausal women were limited by both sample size and the use of assays with low sensitivity and poor reliability $(294,295)$. However, liquid chromatography tandem mass spectrometry assays -which are relatively accurate and provide precise measurements of sex steroid hormones than prior immunoassay methods - confirm lower levels of testosterone levels in postmenopausal women (38) and also among women with bilateral oophorectomy (296).

The ovary is known to produce testosterone. Laughlin et al (39), analyzed sex steroid hormone levels in 684 women aged 50 to 89 years in the Rancho Bernardo Study, 
and reported reduced levels of both total and bioavailable testosterone by more than $40 \%$ in women with bilateral oophorectomy compared to those with intact ovaries. This association was independent of age and body mass index. Building on that observation, Davison et al (253), in a cross-sectional study of 1,423 healthy women aged 18 to 79 years, demonstrated that levels of total testosterone, calculated free testosterone, dehydroepiandrosterone sulfate, and androstenedione declined steeply with age. Moreover, in women who were 55 years or older, those who reported a history of bilateral oophorectomy had lower androgen levels (total and calculated free testosterone) compared to similar aged women with intact ovaries. This study further demonstrated that decline in testosterone levels begins before women arrive at menopause and that the postmenopausal ovary is ongoing site of testosterone production (253). Measuring androgens using ultrasensitive assays in 347 community-dwelling women aged 65 to 98 years enrolled in the Cardiovascular Health Study, Cappola et al (293), also reported that women with bilateral oophorectomy had $23 \%$ lower total testosterone and $16 \%$ lower free testosterone levels than those with at least one intact ovary, while estrogen use, corticosteroid use, and low body mass index were independent predictors of the relationship.

Several studies have identified biochemical and clinical hyperandrogenism in women with diabetes mellitus $(36,82,86)$, however the pathogenesis of this androgen excess in diabetic women is not thoroughly understood. Furthermore, none of the studies investigated androgen levels in diabetic women with oophorectomy. After showing that diabetic women have lower levels of SHBG and elevated non SHBG bound testosterone levels, Korytkowski et al (82), speculated that the ovaries are a source of excess 
androgens in postmenopausal diabetic women. While entirely plausible, no further studies have yet attempted to test this theory. On the other hand, the adrenals may also contribute to hyperandrogenemia in diabetic women. Stress-induced alterations in the hypothalamic-pituitary-adrenal axis which leads to elevations in corticotrophin-releasing factor and corticotrophin could drive an increase in androgen production (82), although, DHEAS levels, an androgen produced by the adrenal cortex, were similar in diabetic and non diabetic women in our study as in previous studies (82).

There are several potential mechanisms for the elevated levels of testosterone postmenopausal diabetic women. In premenopausal women, luteinizing hormone (LH) is known to drive the production of testosterone (297), and increased production of LH in the menopause may stimulate the ovaries to produce more testosterone (35). Diabetic women are insulin resistant, and hyperinsulinemia stimulates ovarian production of testosterone while insulin resistance results in lower levels of SHBG (298), which together may result in increased testosterone in diabetic women (36). Additionally, the ovaries in diabetic women may have reduced ability to convert androgen to estrogen due to reduction of ovarian aromatase activity (94). Evidence obtained from women with polycystic ovary syndrome (PCOS), who are usually insulin resistant and at high risk for glucose intolerance, further suggests an ovarian source of hyperandrogenemia in diabetic women (93). Even when adrenal androgen secretion is suppressed by dexamethasone administration, women with PCOS still exhibit higher androgen levels than normal healthy women, suggestive of an ovarian origin of androgen excess $(299,300)$. Moreover, elevations in luteinizing hormones coupled with hyperinsulinemia work synergistically to increase ovarian enzymatic activity involved in the synthesis of 
testosterone precursors in women with PCOS (301). These observations are also corroborated by evidence from in vitro studies in which cultured theca cells from PCOS women showed increased androgen responsiveness to insulin (300).

In the present study, after adjusting for the effect of age and body mass index, women with diabetes and bilateral oophorectomy, like non-diabetics, had lower levels of total testosterone compared to diabetic women with no oophorectomy or naturally menopausal women without oophorectomy. Taken together, these findings (which also support the first hypothesis of the present study) are interesting since they suggest that oophorectomy moderates levels of testosterone in diabetic women and consequentially confirms an ovarian source of testosterone in postmenopausal diabetic women.

During the menopausal transition, androgen levels remain fairly stable while there is a marked reduction in estradiol levels, resulting in a relatively higher androgenic hormonal milieu in older women (253). It is possible that the prolong exposure to LH and hyperinsulinemia which enhances androgen excess may alter plasma lipid profiles and increase visceral adiposity accumulation to a greater extent in diabetic women, thereby predisposing them to a higher cardiovascular disease risk (82). Accordingly, we hypothesized that oophorectomy will moderate CVD risk in diabetic women by reducing androgen levels. However, in multivariable models comparing diabetic women with oophorectomy to diabetic women without oophorectomy or naturally menopausal women with diabetes, no protective effect for oophorectomy was identified. 


\section{Hypothesis Two}

Contrary to our hypothesis, diabetic women with oophorectomy appeared to have an increased risk for prevalent cardiovascular disease and cardiovascular related mortality. Oophorectomy also appeared to be inversely associated with incident cardiovascular disease in diabetic women, although the available sample size did not provide sufficient statistical power to establish if this association truly exists. While the risk of prevalent CVD was dependent on age at oophorectomy, CVD mortality was independent of surgical removal of the ovaries. This perhaps portrays a complex relationship between ovarian function and cardiovascular disease risk in diabetic women that may not be explained simply by ovarian androgen loss. For example, a U-shaped relationship between testosterone and CHD incidence was observed in a prospective, population-based study of 639 postmenopausal women, with a mean age of 73.8 years who were followed for a median of 12.3 years (266).

Low testosterone may signal systemic illness or an indication of subclinical heart disease (266). The Multi-Ethnic Study of Atherosclerosis, which collected data on 1,947 postmenopausal women aged 45 to 84 years also reported a divergent influence of testosterone on subclinical heart disease (302). While total and bioavailable testosterone were positively associated with carotid artery intimal-medial thickness independent of age, BMI, hypertension, smoking, HDL-cholesterol, LDL-cholesterol and insulin sensitivity, bioavailable testosterone was inversely associated with presence of coronary artery calcium (302). These results may suggest that an optimal range of testosterone may exist for cardiovascular health in women (266). 
One important observation of notable significance is that the prevalence of current hormone therapy use was four times higher in diabetic women with bilateral oophorectomy compared to diabetic women without oophorectomy. Results from the Women's Health Initiative indicate that hormone therapy has an adverse effect on CVD in women with diabetes $(60,303)$. The Estrogen Replacement and Atherosclerosis randomized placebo-controlled trial of 250 postmenopausal women given conjugated equine estrogen with or without medroxyprogesterone acetate, also showed that hormone therapy influenced coronary heart disease progression significantly more in women with diabetes than in women without diabetes (78). This may have driven the apparent increased prevalent as well as incident cardiovascular related mortality risk in diabetic women with oophorectomy. Strikingly, women with diabetes and oophorectomy tended to be more likely to have a family history of myocardial infarction and diabetes which may mean that such select populations may be a high risk population, although the statistically non-significant reduced risk of incident CVD in the SOF study seems to contradict this concept.

\section{Hypothesis Three}

Age at oophorectomy seems to moderate the risk of CVD among diabetic women without ovaries since the elevated risk of CVD was limited to diabetic women with oophorectomy at an earlier age. CVD risk was threefold in diabetic women who had an oophorectomy before age 45 compared to diabetic women who underwent this surgical procedure after age 45 years $\left(\mathrm{p}_{\text {interaction }}=0.001\right)$. Additionally the spline regression demonstrated that bilateral oophorectomy performed before the average age at natural 
menopause increased CVD risk in women with or without diabetes. Conversely, oophorectomy after the average age at natural menopause did not indicate risk or benefit to cardiovascular health in either diabetic or non-diabetic women. This observation was contrary to that observed for age at menopause whereby menopause after age 51 was associated with a statistically significant reduced risk of incident CVD.

. A family history of heart disease was more common among younger diabetic women who had oophorectomy. Our interpretation of these data is that oophorectomy was performed more often in younger women who inherited a risk factor for heart disease. One possibility is the Metabolic Syndrome with PCOS and anovulation. In PCOS women who had developed diabetes, dysfunctional uterine bleeding may have led to hysterectomy with/or without oophorectomy at a young age in this group of women with insulin resistance and heart disease risk. This interpretation of the data would explain the similar CVD risk in women with hysterectomy and oophorectomy compared to those with hysterectomy alone in the WHI study(59), since dysfunctional uterine bleeding may have resulted in hysterectomy with or without oophorectomy, depending on the surgeon and the patient's preference.

It is also possible that the disruption in ovarian function that accompanies oophorectomy in younger women influences their CAD risk. Women who have premature/early physiological menopause have been reported to have more coronary atherosclerosis and an increased risk of CHD $(41,42,47,48)$. In a meta-analysis, Atsma et al (41), reported a $27 \%$ increased risk of cardiovascular disease in women who had early natural menopause compared to women who had menopause at or after age 51 years. Similarly, an early age at menopause among 35,616 naturally postmenopausal 
women in the Nurses' Health Study was associated with a $42 \%$ increased risk of CVD events (46). Additionally, each one year decrease in age at menopause was associated with a $3 \%$ increased risk of CVD (46).

On the other hand, the clinical evidence that oophorectomy leads to an elevated CVD risk is both limited and controversial. Some studies have reported an increased risk of CVD events in women who underwent oophorectomy before age 45 years (or the average age at natural menopause) who did not use postmenopausal estrogen therapy (5153). However, others have not found the CVD risk to increase in women who underwent oophorectomy without hormone therapy use before age 45 years; an observation which is consistent even when the referent group was women with natural menopause (55) or women with hysterectomy but ovarian conservation (59). Some studies have even argued that post-surgical use of estrogens in women who have oophorectomy at an early age may not protect against the progression of atherosclerosis. The Los Angeles Atherosclerosis Study, which assessed the cross-sectional relationship between years since bilateral oophorectomy and common carotid artery intima-media thickness in 269 women aged 45 to 60 , reported that bilateral oophorectomy was associated with increasing atherosclerosis independent of high density lipoprotein or low density lipoprotein cholesterol (297). Interestingly, 90 percent of women with oophorectomy (at an average age of 40 years) had a history of hormone therapy use, which suggests that postsurgical estrogen use may not entirely compensate for the loss of estrogen or loss of the overall imbalance of multiple sex hormones that occur when the ovaries are removed (297).

Although the appropriate age at which oophorectomy should be performed remains controversial, some have even recommend oophorectomy for all women over the 
age of 40 years who undergo hysterectomy (304). While ovarian cancer is a rare disease affecting $1.4 \%$ of women, CVD is the leading cause of morbidity and mortality in women, and among diabetic women, heart disease accounts for $68 \%$ of all deaths (102, 305). Parker et al (304), in their Markov decision analytic model, reported that removal of the ovaries in women younger than 65 years at the time of hysterectomy increased their risk of coronary heart disease mortality while a clear benefit of hysterectomy with ovarian conservation was observed. However after age 65 years, no difference in CHD events was found in women with or without oophorectomy. The probability of surviving to age 80 years after hysterectomy at ages 50 to 54 varied from $62.5 \%$ for ovarian conservation without estrogen therapy to $53.9 \%$ for oophorectomy without estrogen therapy (304). Similar to results from the spline regression, this decision analysis did not find any age at which oophorectomy when performed, provided a protective effect for coronary events.

Several mechanisms may exist through which bilateral oophorectomy increases the risk of CVD in women. Bilateral salpingo oophorectomy causes additional hormonal changes beyond those of natural menopause. In our spline regression analyses, oophorectomy at the time of hysterectomy in premenopausal women was associated with adverse cardiovascular health in both diabetic and non-diabetic women even when postmenopausal hormone use is taken into account. The mechanism by which oophorectomy elevates cardiovascular disease risk in both diabetic and non-diabetic women may be related to an adverse cardiovascular risk profile among women who undergo this surgical procedure (57-59). The abrupt cessation of ovarian function as a consequence of bilateral oophorectomy resulting in decreased endogenous estrogens is 
suggested to adversely influence lipid profiles in premenopausal women, although this has not been consistent across all studies (190). Among premenopausal women who underwent bilateral oophorectomy, a sudden significant increase in total and LDL cholesterol levels was observed three months post-surgery, together with a decrease in HDL cholesterol (306-308). Plasma triglycerides did not change after surgery but estrogen administration was reported to reverse these atherogenic changes in lipid profile (306, 307). Punnonen et al (309), studying 25 women with bilateral oophorectomy followed for up to 7 months, reported no increase in serum cholesterol but an increase in serum triglycerides after surgery which peaked at 1 month and declined to pre-operative levels after 6 months. It should be noted that these changes in lipid profile towards a more atherogenic profile have also been reported in women transitioning naturally through menopause (310).

Another mechanism by which bilateral oophorectomy may influence CVD risk is through changes in body composition including fat distribution. Animal studies report that bilateral oophorectomy increases abdominal fat (250-252), which can be reversed with estrogen therapy $(\mathrm{HT})(251,252)$. In the third National Health and Nutritional Examination Survey (311), obese women who underwent bilateral oophorectomy before 40 years of age had an increased risk of mortality, with the lack of HT use compounding this effect. Since circulating estrogens suppress and androgens stimulate visceral fat accumulation $(234,253)$, and nearly all the estrogens in cycling women are from the ovary, early bilateral oophorectomy may predispose women to adverse cardiovascular health by increasing abdominal fat and its production of adipokines. 
Assessing large artery stiffening, a biomarker of vascular aging which predisposes women to CVD events, Gavin et al (312), reported that carotid artery compliance was lower in women with hysterectomy alone and in women with both hysterectomy and bilateral oophorectomy when compared to women with no hysterectomy. Interestingly, this greater arterial stiffening with hysterectomy in estrogen-deficient postmenopausal women aged 46 to 80 years was not related to an adverse CVD risk profile (312). Additionally, no differences in traditional CVD risk factors (adiposity, blood pressure, lipoproteins, glucose, and insulin) were identified between women with hysterectomy and those without (312). This study (297), challenges the assumption that an adverse CVD risk profile is the culprit for the elevated risk in CVD among oophorectomized women, but offers another biological mechanism by which hysterectomy with bilateral oophorectomy may influence women's cardiovascular health.

Other population studies have also reported no difference in CVD risk profile in women with and without oophorectomy with some even suggesting that women who have oophorectomy have better CVD risk profiles. A Norwegian study (313) including 326 women with oophorectomy and 1,630 age-matched women reported that except for a greater waist circumference, women with oophorectomy had a more favorable coronary heart disease risk profile, including lower Framingham risk scores, more physical activity, lower levels of total cholesterol, higher levels of high-density lipoprotein cholesterol, lower systolic blood pressure, and lower body mass index. Results from the studies by Gavin et al (312) and Michelsen et al (313) seem to contradict the data in the present study (and other previously described observational studies) in which women in NHANES and SOF studies with oophorectomy (with or without diabetes) were found to 
have adverse cardiovascular risk profile. Self-selection of women seeking risk-reducing salpingo-oophorectomy (313), changes in lifestyle after surgery, survival and selection bias (312), may explain why oophorectomy was associated with no difference or a favorable CVD profile in women with oophorectomy compared to age matched women with intact ovaries. For example, Gavin et al (312), included only women (with or without oophorectomy) who were normotensive, nonsmokers, nondiabetic and not taking any lipid lowering therapy, while Michelsen et al (313) sampled women from families with hereditary breast and/or ovarian cancer who had undergone risk-reducing salpingooophorectomy after genetic counseling and were also more likely to establish a healthier lifestyle.

\section{Addressing the conundrum about oophorectomy and women's long term health}

A debate exists regarding the risks and benefits of prophylactic bilateral oophorectomy at the time of hysterectomy. This debate has resulted in several revisions in practice guidelines and recommendations. In 1999, the American College of Obstetricians and Gynecologists stated that, "prophylactic oophorectomy should be based not only on the patient's age but also on other factors that weigh individual risk for developing ovarian cancer against the loss of ovarian function" (204). However this recommendation was revised in 2008 to "strong consideration should be given to retaining normal ovaries in premenopausal women who are not at increased genetic risk of ovarian cancer...Given the risk of ovarian cancer in postmenopausal women, ovarian removal at the time of hysterectomy should be considered for these women" (314). Some 
authors have argued that since there is no effective screening method for ovarian cancer, prophylactic oophorectomy at the time of hysterectomy is a good option to prevent subsequent ovarian cancer which has 22,240 new cases each year $(315,316)$. They further argue that prophylactic oophorectomy is associated with a 80-90\% and 50-60\% reduced risk of ovarian and breast cancer, respectively (317). In high risk women (for example BRCA mutation carriers), these estimates are higher with oophorectomy being associated with an $85 \%$ reduction in BRCA1-associated gynecologic cancer risk as well as a $72 \%$ reduction in BRCA2-associated breast cancer risk (318).

Proponents of ovarian conservation at the time of hysterectomy argue that despite the protective effect of oophorectomy on the development of breast and ovarian cancer, it has detrimental effects on long term health by increasing the risk of all cause mortality (28\%), lung cancer (45\%), coronary heart disease $(33 \%)$, stroke $(62 \%)$, osteoporosis and bone fractures (50\%), cognitive impairment (60\%) and Parkinsonism (80\%) (317). These estimates may even vary depending on the reference population, the age at oophorectomy, and the use of postoperative hormone therapy (317). The cumulative lifetime mortality rate from ovarian cancer for women after hysterectomy for benign disease is usually reported be $1.4 \%$ or 1 in 70 (305). However, figure may be misleading since this estimate is limited to women with BRCA1 or BRCA2 mutations or other highrisk germ-line mutations. In the general female population, the cumulative lifetime mortality from ovarian cancer after hysterectomy for benign disease is $0.47 \%$, or fewer than one in 200 (305). Sightler et al (319) asserted that performing prophylactic oophorectomy in women undergoing hysterectomy for benign conditions at age 40 or older would prevent 1,000 women from getting ovarian cancer each year nationwide. 
Ovarian cancer is the fifth leading cause of death in women, and the leading cause of death from all gynecologic cancers in the United States (316). Although ovarian cancer results in more than 14,030 deaths each year (316), 401,495 women die of cardiovascular disease annually (102). Moreover, no substantial reduction in ovarian cancer mortality is observed when oophorectomy is performed before age 65 since ovarian cancer is a relatively uncommon cause of death in this age group, especially when women with documented germ-line mutations and pedigrees with high risk for ovarian cancer are not considered (304). National estimates show that among women who undergo hysterectomy, the prevalence of concurrent oophorectomy is $38 \%$ in those aged 18 to 44 and 78\% among women aged 55 to 64 years (304). Parker et al (304), demonstrated that hysterectomy with ovarian conservation does reduce the risk of ovarian cancer, with this protective effect persisting for about 20 years. Therefore reducing a woman's risk of ovarian cancer with oophorectomy appears to be outweighed by the increasing likelihood of cardiovascular disease events as well as other chronic conditions (320). In light of this information the decision to remove otherwise healthy ovaries at the time of hysterectomy for benign disease should be approached with great caution especially in younger women since the risk of ovarian cancer in women less than 65 years is minimal compared to cardiovascular diseases.

\section{Strengths and limitations}

This study has several notable strengths. A major one is the approach of three different samples and study designs to investigate the effect of bilateral oophorectomy on cardiovascular health in diabetic women, with serum measures of sex hormones 
evaluated to enhance the biological plausibility of study findings. Additionally, the use of large population-based nationally representative samples/cohort of postmenopausal women increases the generalizability of the findings. These prospective studies were also of sufficient size and duration (> 18 years) to allow associations with incident cardiovascular outcomes to be assessed with good precision. Furthermore, total testosterone was measured by radioimmunoassay after extraction and aluminum oxide column chromatography which exhibits good precision, sensitivity, and high accuracy comparable to liquid chromatography-tandem mass spectrometry, in measuring female androgen concentrations, (297).

However several limitations should be acknowledged. A component of our study used a cross-sectional design, which precluded the determination of temporality. Furthermore, a single measure of sex hormones was used to characterize each woman's hormonal status. Current hormone levels may not adequately reflect earlier levels and a single measure maybe imprecise to some degree (286). However, some studies have demonstrated that a single measurement for plasma sex hormones can reliably categorize average levels over at least a three year period in postmenopausal women, with intraclass correlation coefficients $(0.88,95 \%$ CI:0.84-0.93) not varying by age or time since menopause (321). In contrast to the NHANES studies which defined diabetes using fasting glucose criteria and an oral glucose tolerance test, the SOF study utilized selfreported diabetes mellitus or use of anti-diabetic drugs to diagnose women with diabetes. To add to that, the number of women with cardiovascular disease may be underestimated since information was obtained by self-report of physician diagnosis. Using such criteria may have missed women with subclinical diabetes or CVD. Nevertheless, a significant 
association was found with regards to prevalent and incident cardiovascular disease. In the SOF study, adjustment for lipids in multivariable models was not feasible since only a subsample of women had measures of cholesterol and triglycerides.

Moreover, there is the potential for misclassifications of age at menopause and oophorectomy status due to imprecise recall of events occurring many years before study enrollment and that were not confirmed by medical record review. Women's accuracy in recalling age at oophorectomy seems to surpass recall age at natural menopause with less variance over time; however the accuracy of reported ovarian removal has been observed to be low when compared to medical records (sensitivity:64\% and positive predictive value of $100 \%)(42,322)$. If misclassification by ovarian status was present, it would be expected to bias the finding of this study towards the null. However, since significant effects were identified in all three studies, the probability of such misclassification occurring may be small. Survivor bias may have influenced results especially those from the SOF study since follow-up began long after women underwent oophorectomy. Analytical methods, such as inverse probability weighting to account for competing risks, were not employed because it has been shown in many instances that this selection bias is not severe unless survival is very low in the sampled population (323). Finally, the presence of confounding by indication for gynecological surgery which may predispose some women to a high risk of CVD events cannot be ruled out in the present study. In studies that accounted for the indication of bilateral oophorectomy in multivariable models, no difference with regards to CVD was found between women whose indication for surgery was prophylactic or for benign conditions $(52,311)$. ). It should be noted, 
however, that accounting for confounding by indication using this strategy can only work to the extent that these indications are accurately measured.

\section{Conclusion and recommendations}

While recent years have seen substantial advancements in prevention, early diagnosis and treatment, CVD remains the major cause of death for U. S. adults. However, the prevalence of diabetes, the most important CVD risk factor, is skyrocketing, and imparts a greater risk of CVD in women than men. While the classic risk factors for CVD are similar in both sexes, the hormonal environment, its change with aging, and its connection to body composition differ drastically. Nonetheless, knowledge of how these hormonal differences affect cardiovascular disease in women remains fragmentary. Regrettably, investigations into androgen effects on cardiovascular health in diabetic women are sparse and lacking. Although contentious, current experimental and epidemiologic data suggest that increased testosterone levels may have adverse effects on women's cardiovascular health. Testosterone may have adverse effect on coronary vasculature by increasing macrophage acetylated LDL uptake as well as adhesion of monocytes to the vascular endothelium. However, it is unclear whether these effects occur in vivo. Additionally, testosterone has been found to increase lipogenesis in visceral fat deposits in women.

This present study identified lower levels of total testosterone in diabetic women with bilateral oophorectomy than in those with intact ovaries, but did not find a statistically significant association of free or total testosterone with cardiovascular mortality. Additionally, no protective effect of reduced ovarian testosterone following 
oophorectomy in postmenopausal diabetic women was observed. The main findings of this study do not of course, diminish the important role that androgens may play in cardiovascular health in postmenopausal diabetic women, since hyperandrogenemia is associated with dyslipidemia, hyperinsulinemia, insulin resistance, and increased abdominal fat accumulation, all of which contribute to increasing the risk of cardiovascular disease. Whether androgens adversely affect CVD risk in diabetic women requires more research. Longitudinal investigations are needed into the effect of testosterone on the progression of atherosclerosis in women and to establish the mechanisms by which testosterone affects vascular walls as well as determine testosterone thresholds at which CVD risk is increased. From a clinical perspective, this will aid in identifying high risk postmenopausal women.

Oophorectomy before the average age at natural menopause was found to be associated with an increase risk for cardiovascular events in women with or without diabetes. This result that is consistent with some previous observational and clinical investigations. This study draws attention to the fact that at no age in a woman's life does oophorectomy provide a positive effect on cardiovascular health. Overall, our results convincingly refute the hypothesis that oophorectomy has a protective effect for CVD in women with diabetes. The finding that a strong family history of heart disease, more so than diabetes, might be linked to this CVD risk, and requires further study.

Prophylactic oophorectomy at the time of hysterectomy is a complex decision requiring that the patient and physician consider multiple factors. Concerns about ovarian cancer prevention should be balanced with the increased risk of cardiovascular disease 
and all cause mortality especially among premenopausal women who are at low risk for gynecological cancers.

In conclusion, despite significant advances in preventive and interventional cardiology, cardiovascular disease remains and will continue to be the most important healthcare challenge in industrialized nations due to the increasing obesity epidemic and sedentary lifestyle. The prevalence of CVD is greater in women and since women have a longer longevity than men, they will bear the greatest burden of morbidity and disability related with CVD. Diabetes mellitus amplifies adverse cardiovascular disease outcomes more in women than men. Furthermore, diabetic women have poorer outcomes after coronary interventions such as percutaneous coronary intervention and coronary artery bypass grafting. The prevalence of diabetes is increasing in the United States. In the light of this, it is important and prudent that we understand and identify underlying factors that predispose diabetic women to cardiovascular events to aid in early detection of high risk women in a timely fashion who may benefit from pharmacologic management of their risk factors beyond lifestyle modification. This will have a major impact on cardiovascular health in diabetic women by reducing morbidity and mortality, decreasing healthcare costs, and improving quality of life. 


\section{REFERENCE}

1. Diagnosis and classification of diabetes mellitus. Diabetes care 2004;27 Suppl 1:S5-S10.

2. International Diabetes Federation. Diabetes Atlas, 5th Edition 2012 Update. Brussels, Belgium: International Diabetes Federation, 2012,

3. Centers for Disease Control and Prevention. National Diabetes Fact Sheet: national estimates and general information on diabetes and prediabetes in the United States, 2011. Atlanta, GA: U.S: Department of Health and Human Services, Centers for Disease Control and Prevention,, 2011,

4. Szmuilowicz ED, Stuenkel CA, Seely EW. Influence of menopause on diabetes and diabetes risk. Nature reviews Endocrinology 2009;5(10):553-8.

5. Kaseta JR, Skafar DF, Ram JL, et al. Cardiovascular disease in the diabetic woman. The Journal of clinical endocrinology and metabolism 1999;84(6):18358.

6. Orchard TJ. The impact of gender and general risk factors on the occurrence of atherosclerotic vascular disease in non-insulin-dependent diabetes mellitus. Annals of medicine 1996;28(4):323-33.

7. Lee WL, Cheung AM, Cape D, et al. Impact of diabetes on coronary artery disease in women and men: a meta-analysis of prospective studies. Diabetes care 2000;23(7):962-8. 
8. Kanaya AM, Grady D, Barrett-Connor E. Explaining the sex difference in coronary heart disease mortality among patients with type 2 diabetes mellitus: a meta-analysis. Archives of internal medicine 2002;162(15):1737-45.

9. Juutilainen A, Kortelainen S, Lehto S, et al. Gender difference in the impact of type 2 diabetes on coronary heart disease risk. Diabetes care 2004;27(12):2898904.

10. Hu FB, Stampfer MJ, Solomon CG, et al. The impact of diabetes mellitus on mortality from all causes and coronary heart disease in women: 20 years of follow-up. Archives of internal medicine 2001;161(14):1717-23.

11. Ellis SG. Coronary revascularization for patients with diabetes: updated data favor coronary artery bypass grafting. Journal of the American College of Cardiology 2013;61(8):817-9.

12. Tunstall-Pedoe H. Myth and paradox of coronary risk and the menopause. Lancet 1998;351(9113):1425-7.

13. Weiss AM. Cardiovascular disease in women. Primary care 2009;36(1):73-102, viii.

14. Nanchen D, Rodondi N, Cornuz J, et al. Mortality associated with diabetes and cardiovascular disease in older women. PloS one 2012;7(11):e48818.

15. Huxley R, Barzi F, Woodward M. Excess risk of fatal coronary heart disease associated with diabetes in men and women: meta-analysis of 37 prospective cohort studies. BMJ 2006;332(7533):73-8.

16. Canoy D. Distribution of body fat and risk of coronary heart disease in men and women. Current opinion in cardiology 2008;23(6):591-8. 
17. Canoy D, Boekholdt SM, Wareham N, et al. Body fat distribution and risk of coronary heart disease in men and women in the European Prospective Investigation Into Cancer and Nutrition in Norfolk cohort: a population-based prospective study. Circulation 2007;116(25):2933-43.

18. Ley CJ, Lees B, Stevenson JC. Sex- and menopause-associated changes in bodyfat distribution. The American journal of clinical nutrition 1992;55(5):950-4.

19. Taylor AE, Ebrahim S, Ben-Shlomo Y, et al. Comparison of the associations of body mass index and measures of central adiposity and fat mass with coronary heart disease, diabetes, and all-cause mortality: a study using data from 4 UK cohorts. The American journal of clinical nutrition 2010;91(3):547-56.

20. Zhang C, Rexrode KM, van Dam RM, et al. Abdominal obesity and the risk of all-cause, cardiovascular, and cancer mortality: sixteen years of follow-up in US women. Circulation 2008;117(13):1658-67.

21. Weisfeldt ML, Zieman SJ. Advances in the prevention and treatment of cardiovascular disease. Health Aff (Millwood) 2007;26(1):25-37.

22. Ford ES, Ajani UA, Croft JB, et al. Explaining the decrease in U.S. deaths from coronary disease, 1980-2000. The New England journal of medicine 2007;356(23):2388-98.

23. Roger VL, Go AS, Lloyd-Jones DM, et al. Heart disease and stroke statistics-2012 update: a report from the American Heart Association. Circulation 2012;125(1):e2-e220. 
24. Roger VL, Go AS, Lloyd-Jones DM, et al. Executive summary: heart disease and stroke statistics--2012 update: a report from the American Heart Association. Circulation 2012;125(1):188-97.

25. Sowers JR. Diabetes mellitus and cardiovascular disease in women. Archives of internal medicine 1998;158(6):617-21.

26. Araujo LF, de Matos Soeiro A, Fernandes JL, et al. Coronary artery disease in women: a review on prevention, pathophysiology, diagnosis, and treatment. Vascular health and risk management 2006;2(4):465-75.

27. Shirato S, Swan BA. Women and cardiovascular disease: an evidentiary review. Medsurg nursing : official journal of the Academy of Medical-Surgical Nurses 2010;19(5):282-6, 306.

28. Lokhandwala JO, Skelding KA. Sex differences in percutaneous coronary interventions. Journal of cardiovascular translational research 2009;2(3):300-5.

29. Zbierajewski-Eischeid SJ, Loeb SJ. Myocardial infarction in women: promoting symptom recognition, early diagnosis, and risk assessment. Dimensions of critical care nursing : DCCN 2009;28(1):1-6; quiz 7-8.

30. Xhyheri B, Bugiardini R. Diagnosis and treatment of heart disease: are women different from men? Progress in cardiovascular diseases 2010;53(3):227-36.

31. O'Callaghan KM. Solutions for disparities for women with heart disease. Journal of cardiovascular translational research 2009;2(4):518-25.

32. Anand SS, Xie CC, Mehta S, et al. Differences in the management and prognosis of women and men who suffer from acute coronary syndromes. Journal of the American College of Cardiology 2005;46(10):1845-51. 
33. Fremont AM, Correa-de-Araujo R, Hayes SN. Gender disparities in managed care: it's time for action. Women's health issues : official publication of the Jacobs Institute of Women's Health 2007;17(3):116-9.

34. Vitale C, Miceli M, Rosano GM. Gender-specific characteristics of atherosclerosis in menopausal women: risk factors, clinical course and strategies for prevention. Climacteric : the journal of the International Menopause Society 2007;10 Suppl 2:16-20.

35. Brand JS, van der Schouw YT. Testosterone, SHBG and cardiovascular health in postmenopausal women. International journal of impotence research 2010;22(2):91-104.

36. Andersson B, Marin P, Lissner L, et al. Testosterone concentrations in women and men with NIDDM. Diabetes care 1994;17(5):405-11.

37. Key TJ, Appleby PN, Reeves GK, et al. Circulating sex hormones and breast cancer risk factors in postmenopausal women: reanalysis of 13 studies. British journal of cancer 2011;105(5):709-22.

38. Rothman MS, Carlson NE, Xu M, et al. Reexamination of testosterone, dihydrotestosterone, estradiol and estrone levels across the menstrual cycle and in postmenopausal women measured by liquid chromatography-tandem mass spectrometry. Steroids 2011;76(1-2):177-82.

39. Laughlin GA, Barrett-Connor E, Kritz-Silverstein D, et al. Hysterectomy, oophorectomy, and endogenous sex hormone levels in older women: the Rancho Bernardo Study. The Journal of clinical endocrinology and metabolism 2000;85(2):645-51. 
40. Matthews KA, Crawford SL, Chae CU, et al. Are changes in cardiovascular disease risk factors in midlife women due to chronological aging or to the menopausal transition? Journal of the American College of Cardiology 2009;54(25):2366-73.

41. Atsma F, Bartelink ML, Grobbee DE, et al. Postmenopausal status and early menopause as independent risk factors for cardiovascular disease: a metaanalysis. Menopause 2006;13(2):265-79.

42. Wellons M, Ouyang P, Schreiner PJ, et al. Early menopause predicts future coronary heart disease and stroke: the Multi-Ethnic Study of Atherosclerosis. Menopause 2012;19(10):1081-7.

43. van der Schouw YT, van der Graaf Y, Steyerberg EW, et al. Age at menopause as a risk factor for cardiovascular mortality. The Lancet 1996;347(9003):714-8.

44. Palmer JR, Rosenberg L, Shapiro S. Reproductive factors and risk of myocardial infarction. American journal of epidemiology 1992;136(4):408-16.

45. Cooper GS, Ephross SA, Weinberg CR, et al. Menstrual and reproductive risk factors for ischemic heart disease. Epidemiology 1999;10(3):255-9.

46. Hu FB, Grodstein F, Hennekens CH, et al. Age at natural menopause and risk of cardiovascular disease. Archives of internal medicine 1999;159(10):1061-6.

47. Jacobsen BK, Knutsen SF, Fraser GE. Age at natural menopause and total mortality and mortality from ischemic heart disease: the Adventist Health Study. Journal of clinical epidemiology 1999;52(4):303-7. 
48. Kok HS, van Asselt KM, van der Schouw YT, et al. Heart disease risk determines menopausal age rather than the reverse. Journal of the American College of Cardiology 2006;47(10):1976-83.

49. Whiteman MK, Hillis SD, Jamieson DJ, et al. Inpatient hysterectomy surveillance in the United States, 2000-2004. American journal of obstetrics and gynecology 2008;198(1):34 e1-7.

50. Allison MA, Manson JE, Langer RD, et al. Oophorectomy, hormone therapy, and subclinical coronary artery disease in women with hysterectomy: the Women's Health Initiative coronary artery calcium study. Menopause 2008;15(4 Pt 1):63947.

51. Rocca WA, Grossardt BR, de Andrade M, et al. Survival patterns after oophorectomy in premenopausal women: a population-based cohort study. The lancet oncology 2006;7(10):821-8.

52. Rivera CM, Grossardt BR, Rhodes DJ, et al. Increased cardiovascular mortality after early bilateral oophorectomy. Menopause 2009;16(1):15-23.

53. Parker WH, Broder MS, Chang E, et al. Ovarian conservation at the time of hysterectomy and long-term health outcomes in the nurses' health study. Obstetrics and gynecology 2009;113(5):1027-37.

54. Ingelsson E, Lundholm C, Johansson AL, et al. Hysterectomy and risk of cardiovascular disease: a population-based cohort study. European heart journal 2011;32(6):745-50. 
55. Duan L, Xu X, Koebnick C, et al. Bilateral oophorectomy is not associated with increased mortality: the California Teachers Study. Fertility and sterility 2012;97(1):111-7.

56. Falkeborn M, Schairer C, Naessen T, et al. Risk of myocardial infarction after oophorectomy and hysterectomy. Journal of clinical epidemiology 2000;53(8):832-7.

57. Howard BV, Kuller L, Langer R, et al. Risk of cardiovascular disease by hysterectomy status, with and without oophorectomy: the Women's Health Initiative Observational Study. Circulation 2005;111(12):1462-70.

58. Jacoby VL, Grady D, Sawaya GF. Oophorectomy as a risk factor for coronary heart disease. American journal of obstetrics and gynecology 2009;200(2):140 e19.

59. Jacoby VL, Grady D, Wactawski-Wende J, et al. Oophorectomy vs ovarian conservation with hysterectomy: cardiovascular disease, hip fracture, and cancer in the Women's Health Initiative Observational Study. Archives of internal medicine 2011;171(8):760-8.

60. Rossouw JE, Anderson GL, Prentice RL, et al. Risks and benefits of estrogen plus progestin in healthy postmenopausal women: principal results From the Women's Health Initiative randomized controlled trial. JAMA : the journal of the American Medical Association 2002;288(3):321-33.

61. Anderson GL, Limacher M, Assaf AR, et al. Effects of conjugated equine estrogen in postmenopausal women with hysterectomy: the Women's Health 
Initiative randomized controlled trial. JAMA : the journal of the American Medical Association 2004;291(14):1701-12.

62. Grady D, Herrington D, Bittner V, et al. Cardiovascular disease outcomes during 6.8 years of hormone therapy: Heart and Estrogen/progestin Replacement Study follow-up (HERS II). JAMA : the journal of the American Medical Association 2002;288(1):49-57.

63. Armstrong K, Schwartz JS, Randall T, et al. Hormone replacement therapy and life expectancy after prophylactic oophorectomy in women with BRCA1/2 mutations: a decision analysis. Journal of clinical oncology : official journal of the American Society of Clinical Oncology 2004;22(6):1045-54.

64. Mihmanli V, Mihmanli I, Kantarci F, et al. Carotid pulsatility indices in surgical menopause. Archives of gynecology and obstetrics 2002;266(2):96-100.

65. Prentice RL, Manson JE, Langer RD, et al. Benefits and risks of postmenopausal hormone therapy when it is initiated soon after menopause. American journal of epidemiology 2009;170(1):12-23.

66. Jarvie JL, Foody JM. Recognizing and improving health care disparities in the prevention of cardiovascular disease in women. Current cardiology reports 2010;12(6):488-96.

67. Janssen I, Powell LH, Kazlauskaite R, et al. Testosterone and visceral fat in midlife women: the Study of Women's Health Across the Nation (SWAN) fat patterning study. Obesity (Silver Spring) 2010;18(3):604-10. 
68. Bjorntorp P. The regulation of adipose tissue distribution in humans. International journal of obesity and related metabolic disorders : journal of the International Association for the Study of Obesity 1996;20(4):291-302.

69. Mudali S, Dobs AS, Ding J, et al. Endogenous postmenopausal hormones and serum lipids: the atherosclerosis risk in communities study. The Journal of clinical endocrinology and metabolism 2005;90(2):1202-9.

70. Lambrinoudaki I, Christodoulakos G, Rizos D, et al. Endogenous sex hormones and risk factors for atherosclerosis in healthy Greek postmenopausal women. European journal of endocrinology / European Federation of Endocrine Societies 2006;154(6):907-16.

71. Lear SA, Humphries KH, Kohli S, et al. Visceral adipose tissue, a potential risk factor for carotid atherosclerosis: results of the Multicultural Community Health Assessment Trial (M-CHAT). Stroke; a journal of cerebral circulation 2007;38(9):2422-9.

72. Morley JE, Perry HM, 3rd. Androgens and women at the menopause and beyond. The journals of gerontology Series A, Biological sciences and medical sciences 2003;58(5):M409-16.

73. Torrens JI, Sutton-Tyrrell K, Zhao X, et al. Relative androgen excess during the menopausal transition predicts incident metabolic syndrome in midlife women: study of Women's Health Across the Nation. Menopause 2009;16(2):257-64.

74. Janssen I, Powell LH, Crawford S, et al. Menopause and the metabolic syndrome: the Study of Women's Health Across the Nation. Archives of internal medicine 2008;168(14):1568-75. 
75. Bonds DE, Lasser N, Qi L, et al. The effect of conjugated equine oestrogen on diabetes incidence: the Women's Health Initiative randomised trial. Diabetologia 2006;49(3):459-68.

76. Margolis KL, Bonds DE, Rodabough RJ, et al. Effect of oestrogen plus progestin on the incidence of diabetes in postmenopausal women: results from the Women's Health Initiative Hormone Trial. Diabetologia 2004;47(7):1175-87.

77. Kanaya AM, Herrington D, Vittinghoff E, et al. Glycemic effects of postmenopausal hormone therapy: the Heart and Estrogen/progestin Replacement Study. A randomized, double-blind, placebo-controlled trial. Annals of internal medicine 2003;138(1):1-9.

78. Lamon-Fava S, Herrington DM, Horvath KV, et al. Effect of hormone replacement therapy on plasma lipoprotein levels and coronary atherosclerosis progression in postmenopausal women according to type 2 diabetes mellitus status. Metabolism: clinical and experimental 2010;59(12):1794-800.

79. Stoney RM, Walker KZ, Best JD, et al. Do postmenopausal women with NIDDM have a reduced capacity to deposit and conserve lower-body fat? Diabetes care 1998;21(5):828-30.

80. Zietz B, Cuk A, Hugl S, et al. Association of increased C-peptide serum levels and testosterone in type 2 diabetes. European journal of internal medicine 2000;11(6):322-8.

81. Ding EL, Song Y, Malik VS, et al. Sex differences of endogenous sex hormones and risk of type 2 diabetes: a systematic review and meta-analysis. JAMA : the journal of the American Medical Association 2006;295(11):1288-99. 
82. Korytkowski MT, Krug EI, Daly MA, et al. Does androgen excess contribute to the cardiovascular risk profile in postmenopausal women with type 2 diabetes? Metabolism: clinical and experimental 2005;54(12):1626-31.

83. Goodman-Gruen D, Barrett-Connor E. Sex differences in the association of endogenous sex hormone levels and glucose tolerance status in older men and women. Diabetes care 2000;23(7):912-8.

84. Phillips GB, Tuck CH, Jing TY, et al. Association of hyperandrogenemia and hyperestrogenemia with type 2 diabetes in Hispanic postmenopausal women. Diabetes care 2000;23(1):74-9.

85. Walker KZ, Piers LS, Putt RS, et al. Effects of regular walking on cardiovascular risk factors and body composition in normoglycemic women and women with type 2 diabetes. Diabetes care 1999;22(4):555-61.

86. Tok EC, Ertunc D, Evruke C, et al. The androgenic profile of women with noninsulin-dependent diabetes mellitus. The Journal of reproductive medicine 2004;49(9):746-52.

87. Haffner SM, Dunn JF, Katz MS. Relationship of sex hormone-binding globulin to lipid, lipoprotein, glucose, and insulin concentrations in postmenopausal women. Metabolism: clinical and experimental 1992;41(3):278-84.

88. Karim R, Hodis HN, Stanczyk FZ, et al. Relationship between serum levels of sex hormones and progression of subclinical atherosclerosis in postmenopausal women. The Journal of clinical endocrinology and metabolism 2008;93(1):131-8. 
89. Liu Y, Ding J, Bush TL, et al. Relative androgen excess and increased cardiovascular risk after menopause: a hypothesized relation. American journal of epidemiology 2001;154(6):489-94.

90. Reinecke H, Bogdanski J, Woltering A, et al. Relation of serum levels of sex hormone binding globulin to coronary heart disease in postmenopausal women. The American journal of cardiology 2002;90(4):364-8.

91. Conway GS, Agrawal R, Betteridge DJ, et al. Risk factors for coronary artery disease in lean and obese women with the polycystic ovary syndrome. Clinical endocrinology 1992;37(2):119-25.

92. Talbott E, Guzick D, Clerici A, et al. Coronary heart disease risk factors in women with polycystic ovary syndrome. Arteriosclerosis, thrombosis, and vascular biology 1995;15(7):821-6.

93. Markopoulos MC, Rizos D, Valsamakis G, et al. Hyperandrogenism in women with polycystic ovary syndrome persists after menopause. The Journal of clinical endocrinology and metabolism 2011;96(3):623-31.

94. Stamataki KE, Spina J, Rangou DB, et al. Ovarian function in women with noninsulin dependent diabetes mellitus. Clinical endocrinology 1996;45(5):615-21.

95. Krentz AJ, von Muhlen D, Barrett-Connor E. Searching for polycystic ovary syndrome in postmenopausal women: evidence of a dose-effect association with prevalent cardiovascular disease. Menopause 2007;14(2):284-92.

96. Perez-Lopez FR, Larrad-Mur L, Kallen A, et al. Gender differences in cardiovascular disease: hormonal and biochemical influences. Reprod Sci 2010;17(6):511-31. 
97. Pilote L, Dasgupta K, Guru V, et al. A comprehensive view of sex-specific issues related to cardiovascular disease. CMAJ : Canadian Medical Association journal = journal de l'Association medicale canadienne 2007;176(6):S1-44.

98. Nicholson C. Cardiovascular disease in women. Nurs Stand 2007;21(38):43-7.

99. Ahmed SB, Grace SL, Stelfox HT, et al. Gender bias in cardiovascular advertisements. Journal of evaluation in clinical practice 2004;10(4):531-8.

100. Fact sheet on cardiovascular disease:World Health Organisation (http://www.who.int/topics/cardiovascular_diseases/en/). (Accessed February 21, 2013).

101. Mackay J, Mensah, GA. The atlas of heart disease and stroke. Genova:World Health Organization, 2004.

102. Go AS, Mozaffarian D, Roger VL, et al. Heart disease and stroke statistics--2013 update: a report from the American Heart Association. Circulation 2013;127(1):e6-e245.

103. Dougherty PL, Faucher MA, Gillman PH, et al. Cardiovascular disease. Why women need to get serious about heart health now. AWHONN lifelines / Association of Women's Health, Obstetric and Neonatal Nurses 2006;10(5):390-8.

104. Lloyd-Jones D, Adams R, Carnethon M, et al. Heart disease and stroke statistics-2009 update: a report from the American Heart Association Statistics Committee and Stroke Statistics Subcommittee. Circulation 2009;119(3):480-6.

105. Collins SD, Lansky AJ. Is there a gender disparity in treatment of coronary artery disease? Cardiovascular revascularization medicine : including molecular interventions 2011;12(4):243-6. 
106. Wilkins JT, Ning H, Berry J, et al. Lifetime risk and years lived free of total cardiovascular disease. JAMA : the journal of the American Medical Association 2012;308(17):1795-801.

107. Mosca L, Mochari-Greenberger H, Dolor RJ, et al. Twelve-year follow-up of American women's awareness of cardiovascular disease risk and barriers to heart health. Circulation Cardiovascular quality and outcomes 2010;3(2):120-7.

108. Mosca L, Ferris A, Fabunmi R, et al. Tracking women's awareness of heart disease: an American Heart Association national study. Circulation 2004;109(5):573-9.

109. Kohli P, Gulati M. Exercise stress testing in women: going back to the basics. Circulation 2010;122(24):2570-80.

110. Mujtaba SF, Rizvi SN, Talpur A, et al. Gender based differences in symptoms of acute coronary syndrome. Journal of the College of Physicians and Surgeons-Pakistan : JCPSP 2012;22(5):285-8.

111. Kim ES, Carrigan TP, Menon V. Enrollment of women in National Heart, Lung, and Blood Institute-funded cardiovascular randomized controlled trials fails to meet current federal mandates for inclusion. Journal of the American College of Cardiology 2008;52(8):672-3.

112. Tan YY, Gast GC, van der Schouw YT. Gender differences in risk factors for coronary heart disease. Maturitas 2010;65(2):149-60.

113. Sheifer SE, Canos MR, Weinfurt KP, et al. Sex differences in coronary artery size assessed by intravascular ultrasound. American heart journal 2000;139(4):64953. 
114. Kucher N, Lipp E, Schwerzmann M, et al. Gender differences in coronary artery size per $100 \mathrm{~g}$ of left ventricular mass in a population without cardiac disease. Swiss medical weekly 2001;131(41-42):610-5.

115. Herrington DM, Brown WV, Mosca L, et al. Relationship between arterial stiffness and subclinical aortic atherosclerosis. Circulation 2004;110(4):432-7.

116. Pepine CJ, Kerensky RA, Lambert CR, et al. Some thoughts on the vasculopathy of women with ischemic heart disease. Journal of the American College of Cardiology 2006;47(3 Suppl):S30-5.

117. Quyyumi AA. Women and ischemic heart disease: pathophysiologic implications from the Women's Ischemia Syndrome Evaluation (WISE) Study and future research steps. Journal of the American College of Cardiology 2006;47(3 Suppl):S66-71.

118. Harris DJ, Douglas PS. Enrollment of women in cardiovascular clinical trials funded by the National Heart, Lung, and Blood Institute. The New England journal of medicine 2000;343(7):475-80.

119. Melloni C, Berger JS, Wang TY, et al. Representation of women in randomized clinical trials of cardiovascular disease prevention. Circulation Cardiovascular quality and outcomes 2010;3(2):135-42.

120. Heidenreich PA, Trogdon JG, Khavjou OA, et al. Forecasting the future of cardiovascular disease in the United States: a policy statement from the American Heart Association. Circulation 2011;123(8):933-44. 
121. Trogdon JG, Finkelstein EA, Nwaise IA, et al. The economic burden of chronic cardiovascular disease for major insurers. Health promotion practice $2007 ; 8(3): 234-42$.

122. Mosca L, Linfante AH, Benjamin EJ, et al. National study of physician awareness and adherence to cardiovascular disease prevention guidelines. Circulation 2005;111(4):499-510.

123. Vaccarino V, Badimon L, Corti R, et al. Ischaemic heart disease in women: are there sex differences in pathophysiology and risk factors? Position paper from the working group on coronary pathophysiology and microcirculation of the European Society of Cardiology. Cardiovascular research 2011;90(1):9-17.

124. Ridker PM, Buring JE, Rifai N, et al. Development and validation of improved algorithms for the assessment of global cardiovascular risk in women: the Reynolds Risk Score. JAMA : the journal of the American Medical Association 2007;297(6):611-9.

125. Anand SS, Islam S, Rosengren A, et al. Risk factors for myocardial infarction in women and men: insights from the INTERHEART study. European heart journal 2008;29(7):932-40.

126. Salmon JE, Roman MJ. Subclinical atherosclerosis in rheumatoid arthritis and systemic lupus erythematosus. The American journal of medicine 2008;121(10 Suppl 1):S3-8.

127. Wang TJ. New cardiovascular risk factors exist, but are they clinically useful? European heart journal 2008;29(4):441-4. 
128. Bello N, Mosca L. Epidemiology of coronary heart disease in women. Progress in cardiovascular diseases 2004;46(4):287-95.

129. Willett WC, Green A, Stampfer MJ, et al. Relative and absolute excess risks of coronary heart disease among women who smoke cigarettes. The New England journal of medicine 1987;317(21):1303-9.

130. Prescott E, Hippe M, Schnohr P, et al. Smoking and risk of myocardial infarction in women and men: longitudinal population study. BMJ 1998;316(7137):1043-7.

131. Tanis BC, van den Bosch MA, Kemmeren JM, et al. Oral contraceptives and the risk of myocardial infarction. The New England journal of medicine 2001;345(25):1787-93.

132. He J, Vupputuri S, Allen K, et al. Passive smoking and the risk of coronary heart disease--a meta-analysis of epidemiologic studies. The New England journal of medicine 1999;340(12):920-6.

133. Schenck-Gustafsson K. Risk factors for cardiovascular disease in women. Maturitas 2009;63(3):186-90.

134. The fifth report of the Joint National Committee on Detection, Evaluation, and Treatment of High Blood Pressure (JNC V). Archives of internal medicine 1993;153(2):154-83.

135. Escobar E. Hypertension and coronary heart disease. Journal of human hypertension 2002;16 Suppl 1:S61-3.

136. Finn AV, Nakano M, Narula J, et al. Concept of vulnerable/unstable plaque. Arteriosclerosis, thrombosis, and vascular biology 2010;30(7):1282-92. 
137. James PR, Nelson-Piercy C. Management of hypertension before, during, and after pregnancy. Heart 2004;90(12):1499-504.

138. Craici I, Wagner S, Garovic VD. Preeclampsia and future cardiovascular risk: formal risk factor or failed stress test? Therapeutic advances in cardiovascular disease 2008;2(4):249-59.

139. Duley L. The global impact of pre-eclampsia and eclampsia. Seminars in perinatology 2009;33(3):130-7.

140. Bellamy L, Casas JP, Hingorani AD, et al. Pre-eclampsia and risk of cardiovascular disease and cancer in later life: systematic review and metaanalysis. BMJ 2007;335(7627):974.

141. Hokanson JE, Austin MA. Plasma triglyceride level is a risk factor for cardiovascular disease independent of high-density lipoprotein cholesterol level: a meta-analysis of population-based prospective studies. Journal of cardiovascular risk 1996;3(2):213-9.

142. Castelli WP, Anderson K, Wilson PW, et al. Lipids and risk of coronary heart disease. The Framingham Study. Annals of epidemiology 1992;2(1-2):23-8.

143. Gordon DJ, Probstfield JL, Garrison RJ, et al. High-density lipoprotein cholesterol and cardiovascular disease. Four prospective American studies. Circulation 1989;79(1):8-15.

144. Lewis SJ, Sacks FM, Mitchell JS, et al. Effect of pravastatin on cardiovascular events in women after myocardial infarction: the cholesterol and recurrent events (CARE) trial. Journal of the American College of Cardiology 1998;32(1):140-6. 
145. Mihaylova B, Emberson J, Blackwell L, et al. The effects of lowering LDL cholesterol with statin therapy in people at low risk of vascular disease: metaanalysis of individual data from 27 randomised trials. Lancet 2012;380(9841):581-90.

146. Ridker PM, Danielson E, Fonseca FA, et al. Rosuvastatin to prevent vascular events in men and women with elevated C-reactive protein. The New England journal of medicine 2008;359(21):2195-207.

147. Mora S, Glynn RJ, Hsia J, et al. Statins for the primary prevention of cardiovascular events in women with elevated high-sensitivity C-reactive protein or dyslipidemia: results from the Justification for the Use of Statins in Prevention: An Intervention Trial Evaluating Rosuvastatin (JUPITER) and meta-analysis of women from primary prevention trials. Circulation 2010;121(9):1069-77.

148. Barter P. The role of HDL-cholesterol in preventing atherosclerotic disease. European Heart Journal Supplements 2005;7(suppl F):F4-F8.

149. Babelova A, Sedding DG, Brandes RP. Anti-atherosclerotic mechanisms of statin therapy. Current opinion in pharmacology 2013.

150. Dalton M, Cameron AJ, Zimmet PZ, et al. Waist circumference, waist-hip ratio and body mass index and their correlation with cardiovascular disease risk factors in Australian adults. Journal of internal medicine 2003;254(6):555-63.

151. Wormser D, Kaptoge S, Di Angelantonio E, et al. Separate and combined associations of body-mass index and abdominal adiposity with cardiovascular disease: collaborative analysis of 58 prospective studies. Lancet 2011;377(9771):1085-95. 
152. Bogers RP, Bemelmans WJ, Hoogenveen RT, et al. Association of overweight with increased risk of coronary heart disease partly independent of blood pressure and cholesterol levels: a meta-analysis of 21 cohort studies including more than 300000 persons. Archives of internal medicine 2007;167(16):1720-8.

153. Wilson PW, D'Agostino RB, Sullivan L, et al. Overweight and obesity as determinants of cardiovascular risk: the Framingham experience. Archives of internal medicine 2002;162(16):1867-72.

154. Morse SA, Gulati R, Reisin E. The obesity paradox and cardiovascular disease. Current hypertension reports 2010;12(2):120-6.

155. Fonarow GC, Srikanthan P, Costanzo MR, et al. An obesity paradox in acute heart failure: analysis of body mass index and inhospital mortality for 108,927 patients in the Acute Decompensated Heart Failure National Registry. American heart journal 2007;153(1):74-81.

156. Galal W, van Gestel YR, Hoeks SE, et al. The obesity paradox in patients with peripheral arterial disease. Chest 2008;134(5):925-30.

157. Diercks DB, Roe MT, Mulgund J, et al. The obesity paradox in non-ST-segment elevation acute coronary syndromes: results from the Can Rapid risk stratification of Unstable angina patients Suppress ADverse outcomes with Early implementation of the American College of Cardiology/American Heart Association Guidelines Quality Improvement Initiative. American heart journal 2006;152(1):140-8. 
158. Strandberg TE, Strandberg AY, Salomaa VV, et al. Explaining the obesity paradox: cardiovascular risk, weight change, and mortality during long-term follow-up in men. European heart journal 2009;30(14):1720-7.

159. Goodpaster BH, Delany JP, Otto AD, et al. Effects of diet and physical activity interventions on weight loss and cardiometabolic risk factors in severely obese adults: a randomized trial. JAMA : the journal of the American Medical Association 2010;304(16):1795-802.

160. Manson JE, Hu FB, Rich-Edwards JW, et al. A prospective study of walking as compared with vigorous exercise in the prevention of coronary heart disease in women. The New England journal of medicine 1999;341(9):650-8.

161. Manson JE, Greenland P, LaCroix AZ, et al. Walking compared with vigorous exercise for the prevention of cardiovascular events in women. The New England journal of medicine 2002;347(10):716-25.

162. Van Gaal LF, Mertens IL, De Block CE. Mechanisms linking obesity with cardiovascular disease. Nature 2006;444(7121):875-80.

163. Fox CS, Pencina MJ, Wilson PW, et al. Lifetime risk of cardiovascular disease among individuals with and without diabetes stratified by obesity status in the Framingham heart study. Diabetes care 2008;31(8):1582-4.

164. Economic Costs of Diabetes in the U.S. in 2012. Diabetes care 2013.

165. Saltiki K, Cimponeriu A, Lili K, et al. Severity of coronary artery disease in postmenopausal diabetic women. Hormones (Athens) 2008;7(2):148-55. 
166. Gregg EW, Cheng YJ, Saydah S, et al. Trends in death rates among U.S. adults with and without diabetes between 1997 and 2006: findings from the National Health Interview Survey. Diabetes care 2012;35(6):1252-7.

167. Gregg EW, Gu Q, Cheng YJ, et al. Mortality trends in men and women with diabetes, 1971 to 2000. Annals of internal medicine 2007;147(3):149-55.

168. Kramer HU, Raum E, Ruter G, et al. Gender disparities in diabetes and coronary heart disease medication among patients with type 2 diabetes: results from the DIANA study. Cardiovascular diabetology 2012;11:88.

169. Fu AZ, Zhang Q, Davies MJ, et al. Underutilization of statins in patients with type 2 diabetes in US clinical practice: a retrospective cohort study. Current medical research and opinion 2011;27(5):1035-40.

170. Franzini L, Ardigo D, Cavalot F, et al. Women show worse control of type 2 diabetes and cardiovascular disease risk factors than men: Results from the MIND.IT Study Group of the Italian Society of Diabetology. Nutrition, metabolism, and cardiovascular diseases : NMCD 2012.

171. Blondal M, Ainla T, Marandi T, et al. Sex-specific outcomes of diabetic patients with acute myocardial infarction who have undergone percutaneous coronary intervention: a register linkage study. Cardiovascular diabetology 2012;11:96.

172. Bigley MB. Diabetes: A public health pandemic. In: Battle C, ed. Essentials of Public Health Biology. Sudbury MA: Jones and Bartlett Publishers 2008:449-58.

173. Buse JB, Polonsky KS, Burant CF. Type 2 diabetes mellitus. In: Melmed S, Polonsky KS, Larsen PR, et al., eds. Williams Textbook of Endocrinology: Expert Consult. Philadelphia: Elsevier/Saunders, 2011:1371 - 435. 
174. van Tilburg J, van Haeften TW, Pearson P, et al. Defining the genetic contribution of type 2 diabetes mellitus. Journal of medical genetics 2001;38(9):569-78.

175. Metcalfe KA, Hitman GA, Rowe RE, et al. Concordance for type 1 diabetes in identical twins is affected by insulin genotype. Diabetes care 2001;24(5):838-42.

176. Raffel LJ, Rotter JI. Type 1 Diabetes. In: King RA, Rotter JI, Motulsky AG, eds. The Genetic Basis of Common Diseases. New York: Oxford University Press, 2002:431-56.

177. Kaku K. Pathophysiology of type 2 diabetes and its treatment policy. JMAJ 2010;53(Suppl 1):41-6.

178. Qu H-Q, Polychronakos C. The Genetic Basis of Diabetes In: Tsatsoulis A, Wyckoff J, Brown FM, eds. Diabetes in Women: Pathophysiology and Therapy. New York: Humana Press, 2010:377-414.

179. Westphal SA. Obesity, abdominal obesity, and insulin resistance. Clinical cornerstone 2008;9(1):23-9; discussion 30-1.

180. Muacevic-Katanec D, Reiner Z. Diabetic dyslipidemia or 'diabetes lipidus'? Expert review of cardiovascular therapy 2011;9(3):341-8.

181. Claus TH, Lowe DB, Liang Y, et al. Specific inhibition of hormone-sensitive lipase improves lipid profile while reducing plasma glucose. The Journal of pharmacology and experimental therapeutics 2005;315(3):1396-402.

182. Gajula S, Reddy A, Kurukulasuriya R, et al. Cardiovascular Disease in Women With Diabetes. In: Tsatsoulis A, Wyckoff J, Brown FM, eds. Diabetes in Women: Pathophysiology and Therapy. New York: Humana Press, 2010:35-56. 
183. Ferreiro JL, Gomez-Hospital JA, Angiolillo DJ. Platelet abnormalities in diabetes mellitus. Diabetes \& vascular disease research : official journal of the International Society of Diabetes and Vascular Disease 2010;7(4):251-9.

184. The vascular system In: Sanders T, Scanlon V, eds. Essentials of Anatomy and Physiology. Philadelphia, PA: F.A. Davis Company, 2007:290-319.

185. Hill K. The demography of menopause. Maturitas 1996;23(2):113-27.

186. Lawlor DA, Ebrahim S, Davey Smith G. Role of endogenous oestrogen in aetiology of coronary heart disease: analysis of age related trends in coronary heart disease and breast cancer in England and Wales and Japan. BMJ 2002;325(7359):311-2.

187. Vaidya D, Becker DM, Bittner V, et al. Ageing, menopause, and ischaemic heart disease mortality in England, Wales, and the United States: modelling study of national mortality data. BMJ 2011;343:d5170.

188. Witteman JC, Moerman CJ, Westendorp IC. Myth of the menopause paradox. Lancet 1998;352(9125):407.

189. Stuenkel CA. Cardiovascular risk and early menopause: cause or consequence? Menopause 2012;19(10):1067-9.

190. Godsland IF, Wynn V, Crook D, et al. Sex, plasma lipoproteins, and atherosclerosis: prevailing assumptions and outstanding questions. American heart journal 1987;114(6):1467-503.

191. Archer DF. Premature menopause increases cardiovascular risk. Climacteric : the journal of the International Menopause Society 2009;12 Suppl 1:26-31. 
192. Gordon T, Kannel WB, Hjortland MC, et al. Menopause and coronary heart disease. The Framingham Study. Annals of internal medicine 1978;89(2):157-61.

193. Colditz GA, Willett WC, Stampfer MJ, et al. Menopause and the risk of coronary heart disease in women. The New England journal of medicine 1987;316(18):1105-10.

194. Furman RH. Are gonadal hormones (estrogens and androgens) of significance in the development of ischemic heart disease. Annals of the New York Academy of Sciences 1968;149(2):822-33.

195. Bittner V. Menopause, age, and cardiovascular risk: a complex relationship. Journal of the American College of Cardiology 2009;54(25):2374-5.

196. Zago V, Sanguinetti S, Brites F, et al. Impaired high density lipoprotein antioxidant activity in healthy postmenopausal women. Atherosclerosis 2004;177(1):203-10.

197. Tremollieres FA, Pouilles JM, Ribot CA. Relative influence of age and menopause on total and regional body composition changes in postmenopausal women. American journal of obstetrics and gynecology 1996;175(6):1594-600.

198. Blouin K, Boivin A, Tchernof A. Androgens and body fat distribution. The Journal of steroid biochemistry and molecular biology 2008;108(3-5):272-80.

199. Guthrie JR, Dennerstein L, Taffe JR, et al. Central abdominal fat and endogenous hormones during the menopausal transition. Fertility and sterility $2003 ; 79(6): 1335-40$.

200. Li C, Ford ES, McGuire LC, et al. Increasing trends in waist circumference and abdominal obesity among US adults. Obesity (Silver Spring) 2007;15(1):216-24. 
201. Domchek SM, Rebbeck TR. Prophylactic oophorectomy in women at increased cancer risk. Current opinion in obstetrics \& gynecology 2007;19(1):27-30.

202. Lowder JL, Oliphant SS, Ghetti C, et al. Prophylactic bilateral oophorectomy or removal of remaining ovary at the time of hysterectomy in the United States, 1979-2004. American journal of obstetrics and gynecology 2010;202(6):538 e1-9.

203. Merrill RM. Hysterectomy surveillance in the United States, 1997 through 2005. Medical science monitor : international medical journal of experimental and clinical research 2008;14(1):CR24-31.

204. Novetsky AP, Boyd LR, Curtin JP. Trends in bilateral oophorectomy at the time of hysterectomy for benign disease. Obstetrics and gynecology 2011;118(6):12806.

205. Jacoby VL, Vittinghoff E, Nakagawa S, et al. Factors associated with undergoing bilateral salpingo-oophorectomy at the time of hysterectomy for benign conditions. Obstetrics and gynecology 2009;113(6):1259-67.

206. Munson BL. Myths and facts...about polycystic ovarian syndrome. Nursing 2002;32(11 Pt 1):78.

207. Iftikhar S, Collazo-Clavell ML, Roger VL, et al. Risk of cardiovascular events in patients with polycystic ovary syndrome. The Netherlands journal of medicine 2012;70(2):74-80.

208. Atkin S. Cardiovascular disease in polycystic ovary syndrome. Clinical endocrinology 2013. 
209. de Groot PC, Dekkers OM, Romijn JA, et al. PCOS, coronary heart disease, stroke and the influence of obesity: a systematic review and meta-analysis. Human reproduction update 2011;17(4):495-500.

210. Christian RC, Dumesic DA, Behrenbeck T, et al. Prevalence and predictors of coronary artery calcification in women with polycystic ovary syndrome. The Journal of clinical endocrinology and metabolism 2003;88(6):2562-8.

211. Talbott EO, Guzick DS, Sutton-Tyrrell K, et al. Evidence for association between polycystic ovary syndrome and premature carotid atherosclerosis in middle-aged women. Arteriosclerosis, thrombosis, and vascular biology 2000;20(11):2414-21.

212. Wang ET, Ku IA, Shah SJ, et al. Polycystic ovary syndrome is associated with higher left ventricular mass index: the CARDIA women's study. The Journal of clinical endocrinology and metabolism 2012;97(12):4656-62.

213. Shaw LJ, Bairey Merz CN, Azziz R, et al. Postmenopausal women with a history of irregular menses and elevated androgen measurements at high risk for worsening cardiovascular event-free survival: results from the National Institutes of Health--National Heart, Lung, and Blood Institute sponsored Women's Ischemia Syndrome Evaluation. The Journal of clinical endocrinology and metabolism 2008;93(4):1276-84.

214. Mani H, Levy MJ, Davies MJ, et al. Diabetes and cardiovascular events in women with polycystic ovary syndrome; a 20 years retrospective cohort study. Clinical endocrinology 2012.

215. Vryonidou A, Papatheodorou A, Tavridou A, et al. Association of hyperandrogenemic and metabolic phenotype with carotid intima-media thickness 
in young women with polycystic ovary syndrome. The Journal of clinical endocrinology and metabolism 2005;90(5):2740-6.

216. Cavasin MA, Sankey SS, Yu AL, et al. Estrogen and testosterone have opposing effects on chronic cardiac remodeling and function in mice with myocardial infarction. American journal of physiology Heart and circulatory physiology 2003;284(5):H1560-9.

217. Simpson ER. Sources of estrogen and their importance. The Journal of steroid biochemistry and molecular biology 2003;86(3-5):225-30.

218. Grodin JM, Siiteri PK, MacDonald PC. Source of estrogen production in postmenopausal women. The Journal of clinical endocrinology and metabolism 1973;36(2):207-14.

219. McLachlan JA, Simpson E, Martin M. Endocrine disrupters and female reproductive health. Best practice \& research Clinical endocrinology \& metabolism 2006;20(1):63-75.

220. Adlercreutz H, Gorbach SL, Goldin BR, et al. Estrogen metabolism and excretion in Oriental and Caucasian women. Journal of the National Cancer Institute 1994;86(14):1076-82.

221. Strauss JF. The synthesis and metabolism of steroid hormones. In: Strauss JF, Barbieri RL, eds. Yen \& Jaffe's Reproductive Endocrinology Philadelphia, PA: Saunders, 2009:79-105.

222. Mendelsohn ME, Karas RH. The protective effects of estrogen on the cardiovascular system. The New England journal of medicine 1999;340(23):180111. 
223. Ansbacher R. The pharmacokinetics and efficacy of different estrogens are not equivalent. American journal of obstetrics and gynecology 2001;184(3):255-63.

224. Dubey RK, Imthurn B, Zacharia LC, et al. Hormone replacement therapy and cardiovascular disease: what went wrong and where do we go from here? Hypertension 2004;44(6):789-95.

225. Barrett-Connor E, Grady D. Hormone replacement therapy, heart disease, and other considerations. Annual review of public health 1998;19:55-72.

226. Clarkson TB, Melendez GC, Appt SE. Timing hypothesis for postmenopausal hormone therapy: its origin, current status, and future. Menopause $2013 ; 20(3): 342-53$.

227. Rossouw JE, Prentice RL, Manson JE, et al. Postmenopausal hormone therapy and risk of cardiovascular disease by age and years since menopause. JAMA : the journal of the American Medical Association 2007;297(13):1465-77.

228. Shufelt CL, Johnson BD, Berga SL, et al. Timing of hormone therapy, type of menopause, and coronary disease in women: data from the National Heart, Lung, and Blood Institute-sponsored Women's Ischemia Syndrome Evaluation. Menopause 2011;18(9):943-50.

229. Salpeter SR, Walsh JM, Greyber E, et al. Mortality associated with hormone replacement therapy in younger and older women: a meta-analysis. Journal of general internal medicine 2004;19(7):791-804.

230. Salpeter SR, Walsh JM, Greyber E, et al. Brief report: Coronary heart disease events associated with hormone therapy in younger and older women. A metaanalysis. Journal of general internal medicine 2006;21(4):363-6. 
231. Salpeter SR, Cheng J, Thabane L, et al. Bayesian meta-analysis of hormone therapy and mortality in younger postmenopausal women. The American journal of medicine 2009;122(11):1016-22 e1.

232. Schierbeck LL, Rejnmark L, Tofteng CL, et al. Effect of hormone replacement therapy on cardiovascular events in recently postmenopausal women: randomised trial. BMJ 2012;345:e6409.

233. Burger HG. Androgen production in women. Fertility and sterility 2002;77 Suppl 4:S3-5.

234. Fogle RH, Stanczyk FZ, Zhang X, et al. Ovarian androgen production in postmenopausal women. The Journal of clinical endocrinology and metabolism 2007;92(8):3040-3.

235. Davey DA. Androgens in women before and after the menopause and post bilateral oophorectomy: clinical effects and indications for testosterone therapy. Womens Health (Lond Engl) 2012;8(4):437-46.

236. Lobo RA. Androgens in postmenopausal women: production, possible role, and replacement options. Obstetrical \& gynecological survey 2001;56(6):361-76.

237. Guzick DS, Wing R, Smith D, et al. Endocrine consequences of weight loss in obese, hyperandrogenic, anovulatory women. Fertility and sterility 1994;61(4):598-604.

238. Liedtke S, Schmidt ME, Vrieling A, et al. Postmenopausal sex hormones in relation to body fat distribution. Obesity (Silver Spring) 2012;20(5):1088-95. 
239. Sowers MF, Beebe JL, McConnell D, et al. Testosterone concentrations in women aged 25-50 years: associations with lifestyle, body composition, and ovarian status. American journal of epidemiology 2001;153(3):256-64.

240. Abdulnour J, Doucet E, Brochu M, et al. The effect of the menopausal transition on body composition and cardiometabolic risk factors: a Montreal-Ottawa New Emerging Team group study. Menopause 2012;19(7):760-7.

241. Goodfriend TL, Kelley DE, Goodpaster BH, et al. Visceral obesity and insulin resistance are associated with plasma aldosterone levels in women. Obesity research 1999;7(4):355-62.

242. Ibrahim MM. Subcutaneous and visceral adipose tissue: structural and functional differences. Obesity reviews : an official journal of the International Association for the Study of Obesity 2010;11(1):11-8.

243. Kalyani RR, Franco M, Dobs AS, et al. The association of endogenous sex hormones, adiposity, and insulin resistance with incident diabetes in postmenopausal women. The Journal of clinical endocrinology and metabolism 2009;94(11):4127-35.

244. Lemieux I, Pascot A, Prud'homme D, et al. Elevated C-reactive protein: another component of the atherothrombotic profile of abdominal obesity. Arteriosclerosis, thrombosis, and vascular biology 2001;21(6):961-7.

245. Yusuf S, Hawken S, Ounpuu S, et al. Effect of potentially modifiable risk factors associated with myocardial infarction in 52 countries (the INTERHEART study): case-control study. Lancet 2004;364(9438):937-52. 
246. Carr MC, Brunzell JD. Abdominal obesity and dyslipidemia in the metabolic syndrome: importance of type 2 diabetes and familial combined hyperlipidemia in coronary artery disease risk. The Journal of clinical endocrinology and metabolism 2004;89(6):2601-7.

247. Fontana L, Eagon JC, Trujillo ME, et al. Visceral fat adipokine secretion is associated with systemic inflammation in obese humans. Diabetes 2007;56(4):1010-3.

248. Ronti T, Lupattelli G, Mannarino E. The endocrine function of adipose tissue: an update. Clinical endocrinology 2006;64(4):355-65.

249. Peverill RE, Teede HJ, Malan E, et al. Relationship of waist and hip circumference with coagulation and fibrinolysis in postmenopausal women. Clin Sci (Lond) 2007;113(9):383-91.

250. Damirchi A, Mehdizade R, Ansar MM, et al. Effects of aerobic exercise training on visceral fat and serum adiponectin concentration in ovariectomized rats. Climacteric : the journal of the International Menopause Society 2010;13(2):1718.

251. Saengsirisuwan V, Pongseeda S, Prasannarong M, et al. Modulation of insulin resistance in ovariectomized rats by endurance exercise training and estrogen replacement. Metabolism: clinical and experimental 2009;58(1):38-47.

252. Babaei P, Mehdizadeh R, Ansar MM, et al. Effects of ovariectomy and estrogen replacement therapy on visceral adipose tissue and serum adiponectin levels in rats. Menopause international 2010;16(3):100-4. 
253. Davison SL, Bell R, Donath S, et al. Androgen levels in adult females: changes with age, menopause, and oophorectomy. The Journal of clinical endocrinology and metabolism 2005;90(7):3847-53.

254. Lovejoy JC, Bray GA, Bourgeois MO, et al. Exogenous androgens influence body composition and regional body fat distribution in obese postmenopausal women-a clinical research center study. The Journal of clinical endocrinology and metabolism 1996;81(6):2198-203.

255. LaRosa JC. Androgens and women's health: genetic and epidemiologic aspects of lipid metabolism. The American journal of medicine 1995;98(1A):22S-6S.

256. Stork S, Bots ML, Grobbee DE, et al. Endogenous sex hormones and C-reactive protein in healthy postmenopausal women. Journal of internal medicine 2008;264(3):245-53.

257. Maturana MA, Breda V, Lhullier F, et al. Relationship between endogenous testosterone and cardiovascular risk in early postmenopausal women. Metabolism: clinical and experimental 2008;57(7):961-5.

258. Ling S, Komesaroff PA, Sudhir K. Cardiovascular physiology of androgens and androgen testosterone therapy in postmenopausal women. Endocrine, metabolic \& immune disorders drug targets 2009;9(1):29-37.

259. McCrohon JA, Jessup W, Handelsman DJ, et al. Androgen exposure increases human monocyte adhesion to vascular endothelium and endothelial cell expression of vascular cell adhesion molecule-1. Circulation 1999;99(17):231722. 
260. Diamanti-Kandarakis E, Lambrinoudaki I, Economou F, et al. Androgens associated with advanced glycation end-products in postmenopausal women. Menopause 2010;17(6):1182-7.

261. Barrett-Connor E, Goodman-Gruen D. Prospective study of endogenous sex hormones and fatal cardiovascular disease in postmenopausal women. BMJ 1995;311(7014):1193-6.

262. Sievers C, Klotsche J, Pieper L, et al. Low testosterone levels predict all-cause mortality and cardiovascular events in women: a prospective cohort study in German primary care patients. European journal of endocrinology / European Federation of Endocrine Societies 2010;163(4):699-708.

263. Rexrode KM, Manson JE, Lee IM, et al. Sex hormone levels and risk of cardiovascular events in postmenopausal women. Circulation 2003;108(14):168893.

264. Braunstein GD, Johnson BD, Stanczyk FZ, et al. Relations between endogenous androgens and estrogens in postmenopausal women with suspected ischemic heart disease. The Journal of clinical endocrinology and metabolism 2008;93(11):426875.

265. Patel SM, Ratcliffe SJ, Reilly MP, et al. Higher serum testosterone concentration in older women is associated with insulin resistance, metabolic syndrome, and cardiovascular disease. The Journal of clinical endocrinology and metabolism 2009;94(12):4776-84. 
266. Laughlin GA, Goodell V, Barrett-Connor E. Extremes of endogenous testosterone are associated with increased risk of incident coronary events in older women. The Journal of clinical endocrinology and metabolism 2010;95(2):740-7.

267. Wehr E, Pilz S, Boehm BO, et al. Low free testosterone levels are associated with all-cause and cardiovascular mortality in postmenopausal diabetic women.

Diabetes care 2011;34(8):1771-7.

268. Haffner SM, Moss SE, Klein BE, et al. Sex hormones and DHEA-SO4 in relation to ischemic heart disease mortality in diabetic subjects. The Wisconsin Epidemiologic Study of Diabetic Retinopathy. Diabetes care 1996;19(10):104550.

269. Centers for Disease Control and Prevention (CDC) National Health and Nutrition Examination Survey: Data Survey Overview. National Center for Health Statistics (NCHS). (http://www.cdc.gov/NCHS/nhanes.htm). (Accessed March 11, 2013).

270. Stark Casagrande S, Fradkin JE, Saydah SH, et al. The Prevalence of Meeting A1C, Blood Pressure, and LDL Goals Among People With Diabetes, 1988-2010. Diabetes care 2013.

271. Report of the expert committee on the diagnosis and classification of diabetes mellitus. Diabetes care 2003;26 Suppl 1:S5-20.

272. Rose GA. The diagnosis of ischaemic heart pain and intermittent claudication in field surveys. Bulletin of the World Health Organization 1962;27:645-58.

273. Annual update of the HHS poverty guidelines--HHS. Notice. Federal register 1995;60(27):7772-4. 
274. Healy GN, Matthews CE, Dunstan DW, et al. Sedentary time and cardiometabolic biomarkers in US adults: NHANES 2003-06. European heart journal 2011;32(5):590-7.

275. Research on the menopause in the 1990s. Report of a WHO Scientific Group. World Health Organization technical report series 1996;866:1-107.

276. Becker A, Bos $\mathrm{G}$, de Vegt F, et al. Cardiovascular events in type 2 diabetes: comparison with nondiabetic individuals without and with prior cardiovascular disease. 10-year follow-up of the Hoorn Study. European heart journal 2003;24(15):1406-13.

277. Dupont WD, Plummer WD. PS power and sample size program available for free on the internet. Controlled clinical trials 1997;18(3):274.

278. Sullivan KM, Dean A, Soe MM. OpenEpi: a web-based epidemiologic and statistical calculator for public health. Public Health Rep 2009;124(3):471-4.

279. Kado DM, Browner WS, Palermo L, et al. Vertebral fractures and mortality in older women: a prospective study. Study of Osteoporotic Fractures Research Group. Archives of internal medicine 1999;159(11):1215-20.

280. Cummings SR, Black DM, Nevitt MC, et al. Appendicular bone density and age predict hip fracture in women. The Study of Osteoporotic Fractures Research Group. JAMA : the journal of the American Medical Association 1990;263(5):665-8.

281. The Study of Osteoporotic Fractures (SOF). (http://sof.ucsf.edu/interface/). (Accessed March 14, 2013). 
282. Ensrud KE, Ewing SK, Fredman L, et al. Circulating 25-hydroxyvitamin D levels and frailty status in older women. The Journal of clinical endocrinology and metabolism 2010;95(12):5266-73.

283. Cummings SR, Lee JS, Lui LY, et al. Sex hormones, risk factors, and risk of estrogen receptor-positive breast cancer in older women: a long-term prospective study. Cancer epidemiology, biomarkers \& prevention : a publication of the American Association for Cancer Research, cosponsored by the American Society of Preventive Oncology 2005;14(5):1047-51.

284. Chapurlat RD, Bauer DC, Cummings SR. Association between endogenous hormones and sex hormone-binding globulin and bone turnover in older women: study of osteoporotic fractures. Bone 2001;29(4):381-7.

285. Cummings SR, Browner WS, Bauer D, et al. Endogenous hormones and the risk of hip and vertebral fractures among older women. Study of Osteoporotic Fractures Research Group. The New England journal of medicine 1998;339(11):733-8.

286. Cauley JA, Lucas FL, Kuller LH, et al. Elevated serum estradiol and testosterone concentrations are associated with a high risk for breast cancer. Study of Osteoporotic Fractures Research Group. Annals of internal medicine 1999;130(4 Pt 1):270-7.

287. SAS. SAS user's guide. Cary, NC: SAS Institute Inc, 2011.

288. Allison PD. Discrete-Time Methods for the Analysis of Event Histories. Sociological Methodology 1982;13:61-98. 
289. Vittinghoff E, McCulloch CE. Relaxing the rule of ten events per variable in logistic and Cox regression. American journal of epidemiology 2007;165(6):7108.

290. Gregory M, Ulmer H, Pfeiffer KP, et al. A set of SAS macros for calculating and displaying adjusted odds ratios (with confidence intervals) for continuous covariates in logistic B-spline regression models. Computer methods and programs in biomedicine 2008;92(1):109-14.

291. Greenland S. Dose-response and trend analysis in epidemiology: alternatives to categorical analysis. Epidemiology 1995;6(4):356-65.

292. Lamarca R, Alonso J, Gomez G, et al. Left-truncated data with age as time scale: an alternative for survival analysis in the elderly population. The journals of gerontology Series A, Biological sciences and medical sciences 1998;53(5):M337-43.

293. Cappola AR, Ratcliffe SJ, Bhasin S, et al. Determinants of serum total and free testosterone levels in women over the age of 65 years. The Journal of clinical endocrinology and metabolism 2007;92(2):509-16.

294. Meldrum DR, Davidson BJ, Tataryn IV, et al. Changes in circulating steroids with aging in postmenopausal women. Obstetrics and gynecology 1981;57(5):624-8.

295. Rozenberg S, Bosson D, Peretz A, et al. Serum levels of gonadotrophins and steroid hormones in the post-menopause and later life. Maturitas 1988;10(3):21524.

296. Bui HN, Struys EA, Martens F, et al. Serum testosterone levels measured by isotope dilution-liquid chromatography-tandem mass spectrometry in 
postmenopausal women versus those in women who underwent bilateral oophorectomy. Annals of clinical biochemistry 2010;47(Pt 3):248-52.

297. de Man M, Derksen E, Pieters G, et al. Cetrorelix suppression test in the diagnostic work-up of severe hyperandrogenism in adolescence. Journal of pediatric endocrinology \& metabolism : JPEM 2008;21(9):905-9.

298. Barbieri RL, Makris A, Randall RW, et al. Insulin stimulates androgen accumulation in incubations of ovarian stroma obtained from women with hyperandrogenism. The Journal of clinical endocrinology and metabolism 1986;62(5):904-10.

299. Lachelin GC, Judd HL, Swanson SC, et al. Long term effects of nightly dexamethasone administration in patients with polycystic ovarian disease. The Journal of clinical endocrinology and metabolism 1982;55(4):768-73.

300. Tosi F, Negri C, Perrone F, et al. Hyperinsulinemia amplifies GnRH agonist stimulated ovarian steroid secretion in women with polycystic ovary syndrome. The Journal of clinical endocrinology and metabolism 2012;97(5):1712-9.

301. Tsilchorozidou T, Overton C, Conway GS. The pathophysiology of polycystic ovary syndrome. Clinical endocrinology 2004;60(1):1-17.

302. Ouyang P, Vaidya D, Dobs A, et al. Sex hormone levels and subclinical atherosclerosis in postmenopausal women: the Multi-Ethnic Study of Atherosclerosis. Atherosclerosis 2009;204(1):255-61.

303. Howard BV, Hsia J, Ouyang P, et al. Postmenopausal hormone therapy is associated with atherosclerosis progression in women with abnormal glucose tolerance. Circulation 2004;110(2):201-6. 
304. Parker WH, Broder MS, Liu Z, et al. Ovarian conservation at the time of hysterectomy for benign disease. Clinical obstetrics and gynecology 2007;50(2):354-61.

305. Parker WH, Broder MS, Berek JS. Hysterectomy sans oophorectomy: The case for leaving a woman's ovaries alone. Contemporary ob/gyn 2006;51(7):38-47

306. Bruschi F, Meschia M, Soma M, et al. Lipoprotein(a) and other lipids after oophorectomy and estrogen replacement therapy. Obstetrics and gynecology 1996;88(6):950-4.

307. Kim CJ, Ryu WS, Kwak JW, et al. Changes in Lp(a) lipoprotein and lipid levels after cessation of female sex hormone production and estrogen replacement therapy. Archives of internal medicine 1996;156(5):500-4.

308. Verhoeven MO, van der Mooren MJ, Teerlink T, et al. The influence of physiological and surgical menopause on coronary heart disease risk markers. Menopause 2009;16(1):37-49.

309. Punnonen R, Rauramo L. Effect of bilateral oophorectomy and peroral estradiol valerate therapy on serum lipids. International journal of gynaecology and obstetrics: the official organ of the International Federation of Gynaecology and Obstetrics 1976;14(1):13-6.

310. Schubert CM, Rogers NL, Remsberg KE, et al. Lipids, lipoproteins, lifestyle, adiposity and fat-free mass during middle age: the Fels Longitudinal Study. Int $J$ Obes (Lond) 2006;30(2):251-60. 
311. McCarthy AM, Menke A, Ouyang P, et al. Bilateral oophorectomy, body mass index, and mortality in u.s. Women aged 40 years and older. Cancer Prev Res (Phila) 2012;5(6):847-54.

312. Gavin KM, Jankowski C, Kohrt WM, et al. Hysterectomy is associated with large artery stiffening in estrogen-deficient postmenopausal women. Menopause 2012;19(9):1000-7.

313. Michelsen TM, Tonstad S, Pripp AH, et al. Coronary heart disease risk profile in women who underwent salpingo-oophorectomy to prevent hereditary breast ovarian cancer. International journal of gynecological cancer : official journal of the International Gynecological Cancer Society 2010;20(2):233-9.

314. ACOG Practice Bulletin No. 89. Elective and risk-reducing salpingooophorectomy. Obstetrics and gynecology 2008;111(1):231-41.

315. Erekson EA, Martin DK, Ratner ES. Oophorectomy: the debate between ovarian conservation and elective oophorectomy. Menopause 2013;20(1):110-4.

316. Ovarian Cancer: National Cancer Institute. (http://www.cancer.gov/cancertopics/types/ovarian). (Accessed April 4, 2013).

317. Rocca WA, Ulrich LG. Oophorectomy for whom and at what age? Primum non nocere. Maturitas 2012;71(1):1-2.

318. Kauff ND, Domchek SM, Friebel TM, et al. Risk-reducing salpingooophorectomy for the prevention of BRCA1- and BRCA2-associated breast and gynecologic cancer: a multicenter, prospective study. Journal of clinical oncology : official journal of the American Society of Clinical Oncology 2008;26(8):13317. 
319. Sightler SE, Boike GM, Estape RE, et al. Ovarian cancer in women with prior hysterectomy: a 14-year experience at the University of Miami. Obstetrics and gynecology 1991;78(4):681-4.

320. Parker WH. Bilateral oophorectomy versus ovarian conservation: effects on longterm women's health. Journal of minimally invasive gynecology 2010;17(2):1616.

321. Hankinson SE, Manson JE, Spiegelman D, et al. Reproducibility of plasma hormone levels in postmenopausal women over a 2-3-year period. Cancer epidemiology, biomarkers \& prevention : a publication of the American Association for Cancer Research, cosponsored by the American Society of Preventive Oncology 1995;4(6):649-54.

322. Phipps AI, Buist DS. Validation of self-reported history of hysterectomy and oophorectomy among women in an integrated group practice setting. Menopause 2009;16(3):576-81.

323. Liu S, Jones RN, Glymour MM. Implications of Lifecourse Epidemiology for Research on Determinants of Adult Disease. Public Health Reviews 2010;32(2):489-511. 


\section{CURRICULUM VITAE}

\section{DUKE APPIAH}

511 South Fifth Street. 1510 Louisville, KY 40202 (219) 6827433 (219) 9229126
Dept. of epidemiology and population health University of Louisville Louisville, KY 40202 d0appi01@ louisville.edu

\section{EDUCATION}

PhD, Epidemiology

University of Louisville, Louisville, KY

Dissertation: Cardiovascular Disease among Diabetic Women with Bilateral Oophorectomy.

Advisor: Carlton A. Hornung, $\mathrm{PhD}, \mathrm{MPH}$.

GPA: $4.0 / 4.0$

\section{MPH, Epidemiology}

University of Louisville, Louisville, KY

Thesis: Highly Active Antiretroviral Therapy Utilization and Mortality: Trends among HIV Outpatients at the University of Louisville: Comparison with the HIV Outpatient Study (HOPS) Advisor: Frank D. Groves, MD, MPH.

GPA: $3.8 / 4.0$

BSc (Hons), Optometry

Kwame Nkrumah University of Science and Technology, Kumasi, Ghana

Honors Thesis: The prevalence of heterophoria in Junior High Schools in the Kumasi metropolis of the Ashanti region

Advisor: Ahmed Abdul-Sadik, OD, PG Dip.

CWA: Second Class upper

Internship: Department of Ophthalmology. Takoradi district hospital, Takoradi, Ghana

\section{Specialty/board certification}

Certification in Public Health, NBPHE-IND-1649 


\section{Graduate Research Assistant}

Dept. of Epidemiology, University of Louisville
$2011-2012$

Supervisor: Dr. Richard Baumgartner

- Assisted in database management and analysis; New Mexico Aging Process Study

- Identified suitable statistical techniques for the analysis of longitudinal data.

- Obtained additional articles for review from electronic and library databases and provided support in the preparation of manuscripts for publication.

\section{ACADEMIC HONORS AND AWARDS}

The Graduate Dean's Citation, University of Louisville

\section{PROFESSIONAL MEMBERSHIP AND SERVICE}

Member, Society for Epidemiologic Research

Member, Kentucky Public Health Association

Member, University of Louisville School of Public Health and Information

Sciences Student Affairs Advisory Committee

Member, University of Louisville School of Public Health and Information

Sciences Student Academic Grievance Committee

Treasurer, Non Traditional Students Unite

\section{Manuscript Reviewer}

Journal of Health Care for the Poor and Underserved
2010 - present

$2007-2012$

$2012-2013$

$2009-2010$

$2008-2009$

\section{COMPUTER SKILLS AND SOFTWARE}

- Skilled in use of Microsoft Office Suite (Word, Excel, Outlook and PowerPoint) as well as EndNote,

- Extensive experience with online databases and advanced search engines (PubMed, OVID, CINAHL)

- Highly experienced with SPSS and SAS for statistical modeling, graphics, and dataset management.

- Moderately proficient in the use of R, for statistical modeling, graphics, and meta-analysis 


\section{PUBLICATIONS}

\section{Published abstracts}

Appiah D, Hornung C, Winters S. Bilateral Oophorectomy and risk of all-cause, cardiovascular disease and cancer mortality. Am. J. Epidemiol. 2012; 175(suppl 11): S41

Appiah D, Winters S, Hornung C. Risks of Cardiovascular Disease among Diabetic Women with Bilateral Oophorectomy. Am. J. Epidemiol. 2011; 173(suppl 11): S97

Appiah D, Groves FD, Huang AK. Mortality Trends among HIV Outpatients at the University of Louisville: Comparison with the HIV Outpatient Study (HOPS) and the Comprehensive Care Center (CCC). Ann Epidemiol. 2010; 20 (9): 712

Appiah D, Kerber RA. Predictors of substance abuse among High School seniors: Findings from Monitoring the Future survey, 1999 - 2008. Am. J. Epidemiol. 2010; 171(suppl 11): S153

\section{PRESENTATIONS}

\section{Platform presentations}

Appiah D, Hornung CA, Winters S. "Bilateral Oophorectomy and risk of all-cause, cardiovascular disease and cancer mortality" June 28, 2012 (Presented at the Annual meeting of the Society for Epidemiologic Research spotlight session titled Matters and Methods of Life and Death: Studies of Mortality, Minneapolis, MN).

Appiah D, Hornung CA, Winters S. "Cardiovascular Disease among Diabetic Women With Surgical Menopause." March 10, 2011 (Presented at the Annual meeting of the Kentucky Public Health Association, Louisville, KY).

Appiah D, Groves FD, Huang AK. "Mortality Trends among HIV Outpatients at the University of Louisville." March 29, 2010 (Presented at the Annual meeting of the Kentucky Public Health Association, Louisville, KY).

\section{Poster presentations}

Harris MJ, Appiah D, Kanotra S. "Access to fruits and vegetables is not sufficient to ensure consumption" October 29, 2012 (Presented at the 140th American Public Health Association Annual Meeting, San Francisco, CA).

Appiah D, Winters S, Hornung CA. "Association of adiposity, fat distribution and body weight trajectories with cardiovascular disease in a national cohort of women with natural and surgical menopause.” September 18, 2012 (Presented at Research!Louisville, Louisville, KY).

Smith M, Appiah D, Woods C. "Alternative vaccination schedules; a role for survival analysis?"April 28, 2012 (Presented at the Pediatric Academic Societies Annual Meeting, Boston, MA). 
Appiah D, Kanotra S. Communities Putting Prevention to Work: Results from pre-intervention survey in Louisville, Kentucky. June 5, 2012 (Presented at the Council for State and Territorial Epidemiologists, Omaha, NE).

Brown-Anderson M, Gholston LR, Appiah D, Howard J, Mayer L. "Tracking behavioral change in community health center dental patients using the Solomon 4-Group Design" October 30, 2011(Presented at the 139th American Public Health Association Annual Meeting, Washington, DC).

Appiah D, Winters S, Hornung CA. "Risks of Cardiovascular Disease among Diabetic Women with Bilateral Oophorectomy." June 22, 2011 (Presented at the North America congress of epidemiology, Montreal, Canada).

Appiah D, Kerber RA. "Predictors of substance abuse among High School seniors: Findings from Monitoring the Future survey, 1999 - 2008." March 10, 2011 (Presented at the Annual meeting of the Kentucky Public Health Association, Louisville, KY).

Appiah D, Groves FD, Huang AK. "Mortality Trends among HIV Outpatients at the University of Louisville: Comparison with the HIV Outpatient Study (HOPS) and the Comprehensive Care Center (CCC)." September 12, 2010 (Presented at the Annual meeting of the American College of Epidemiology, San Francisco, CA).

Appiah D, Kerber RA. "Predictors of substance abuse among High School seniors: Findings from Monitoring the Future survey, 1999 - 2008." June 25, 2010 (Presented at the Annual meeting of the Society for Epidemiologic Research, Seattle, WA).

Appiah D, Groves FD, Huang AK. "Mortality Trends among HIV Outpatients at the University of Louisville: Comparison with the HIV Outpatient Study (HOPS) and the Comprehensive Care Center (CCC)." March 29, 2010 (Presented the Annual meeting of the Kentucky Public Health Association, Louisville, KY). 